\title{
Rotation of Low-mass Stars in Taurus with K2
}

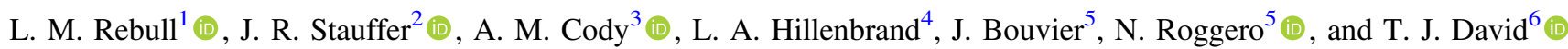 \\ ${ }^{1}$ Infrared Science Archive (IRSA), IPAC, 1200 E. California Blvd., California Institute of Technology, Pasadena, CA 91125, USA; rebull@ipac.caltech.edu \\ ${ }^{2}$ Spitzer Science Center (SSC), (IPAC), 1200 E. California Blvd., California Institute of Technology, Pasadena, CA 9112, USA \\ ${ }^{3}$ Bay Area Environmental Research Institute, 625 2nd St. Ste. 209, Petaluma, CA 94952, USA \\ ${ }^{4}$ Astronomy Department, California Institute of Technology, Pasadena, CA 91125, USA \\ ${ }^{5}$ Université de Grenoble, Institut de Planétologie et d'Astrophysique de Grenoble (IPAG), F-38000 Grenoble, France; CNRS, IPAG, F-38000 Grenoble, France \\ ${ }^{6}$ Center for Computational Astrophysics, Flatiron Institute, New York, NY 10010, USA \\ Received 2020 February 24; revised 2020 April 7; accepted 2020 April 8; published 2020 May 26
}

\begin{abstract}
We present an analysis of K2 light curves (LCs) from Campaigns 4 and 13 for members of the young ( 3 Myr) Taurus association, in addition to an older $(\sim 30 \mathrm{Myr})$ population of stars that is largely in the foreground of the Taurus molecular clouds. Out of 156 of the highest-confidence Taurus members, we find that $81 \%$ are periodic. Our sample of young foreground stars is biased and incomplete, but nearly all stars $(37 / 38)$ are periodic. The overall distribution of rotation rates as a function of color (a proxy for mass) is similar to that found in other clusters: the slowest rotators are among the early M spectral types, with faster rotation toward both earlier FGK and later $\mathrm{M}$ types. The relationship between period and color/mass exhibited by older clusters such as the Pleiades is already in place by Taurus age. The foreground population has very few stars but is consistent with the USco and Pleiades period distributions. As found in other young clusters, stars with disks rotate on average slower, and few with disks are found rotating faster than $\sim 2$ days. The overall amplitude of the LCs decreases with age, and highermass stars have generally lower amplitudes than lower-mass stars. Stars with disks have on average larger amplitudes than stars without disks, though the physical mechanisms driving the variability and the resulting LC morphologies are also different between these two classes.
\end{abstract}

Unified Astronomy Thesaurus concepts: Star formation (1569); Stellar rotation (1629); Starspots (1572)

Supporting material: machine-readable tables

\section{Introduction}

The Taurus-Auriga star-forming region has fundamentally shaped our understanding of how low-mass stars form (see, e.g., Kenyon \& Hartmann 1995). At an age of $\lesssim 3$ Myr (e.g., Kraus \& Hillenbrand 2009; Luhman 2018) and a distance of 140 pc (e.g., Esplin \& Luhman 2017), even the low-mass objects (mid-M to early-L) are bright enough to be well studied. A significant fraction of the members have an infrared (IR) excesses indicative of circumstellar disks.

From the earliest studies of the prototype $\mathrm{T}$ Tauri and other stars in Taurus, variability was included as a defining characteristic of young stars (Joy 1945). The variability arises from starspots, flares, disk interactions, disk accretion, disk structure occulting the star, and more. NASA's K2 mission (Howell et al. 2014) has recently provided high-quality, long duration ( $\sim 70$ days), high cadence ( $\sim 30$ minutes) light curves (LCs) for stars in many different clusters. In two of K2's Campaigns (4 and 13), members of the Taurus-Auriga molecular cloud population were included. Those Taurus members, along with the $\rho$ Oph members observed in K2's Campaign 2 (e.g., Rebull et al. 2018), are the youngest cluster stars observed with $\mathrm{K} 2$.

Aperiodic variability is expected and common in young stars (see, e.g., Cody et al. 2014; Cody \& Hillenbrand 2018). However, when the variability is periodic, we can infer the rotation rate of the star, and hence, we can explore the rotation rates of young stars as a function of stellar mass and disk presence. When combined with comparable observations of other clusters, we can study trends as a function of age. In this paper, we explore the periodic variability of the Taurus members observed with K2. A. M. Cody et al. (2020, in preparation) will explore the range of LC properties of the disked members.

We have previously published our analysis of the K2 data for the Pleiades ( 125 Myr; Rebull et al. 2016a, 2016b; Stauffer et al. 2016b; Papers I, II, and III, respectively), Praesepe ( 790 Myr; Rebull et al. 2017; Paper IV), and Upper Sco/ $\rho$ Oph ( $~ 8$ and $\sim 1$ Myr; Rebull et al. 2018; Paper V). We have deliberately performed our analyses of these clusters, now including Taurus, in a very homogeneous fashion in order to allow for the best intercomparison of the rotation data across the full age range observed with K2. Upper Sco has less reddening and fewer disks than Taurus; $\rho$ Oph has more reddening than, and a comparable disk fraction to, Taurus. While there are nearly 1000 member stars with periodic LCs in each of the Pleiades, Praesepe, and Upper Sco, there are far fewer stars with suitable K2 data at the youngest ages. In $\rho$ Oph, there are 174 member LCs, $106(\sim 60 \%)$ of which are periodic. There are comparable numbers in Taurus, where there are 156 highest-quality members (see Section 2.2 and Table 1 below), and $81 \%$ (127) of those are periodic. In context with the older clusters, Taurus and $\rho$ Oph seem to suffer in comparison, because of fewer stars, more stochastic contributions to the LCs (from disks and accretion, generally yielding a lower fraction of periodic LCs), more reddening and spatial variability in reddening (affecting the scatter in the diagrams), and more uncertainty in membership (yielding a higher contamination rate).

In Section 2, we summarize the data we amassed, including information about the $\mathrm{K} 2$ data, literature information, member selection, dereddening, and disk identification. Section 3 begins with period identification and interpretation as well as a 
Table 1

Summary of Statistics on Taurus Sample ${ }^{a}$

\begin{tabular}{|c|c|c|c|c|c|c|}
\hline Property & $\begin{array}{c}\text { High-confidence } \\
\text { Members }\end{array}$ & $\begin{array}{l}\text { Possible } \\
\text { Members }\end{array}$ & $\begin{array}{l}\sim 3 \mathrm{Myr}^{\mathrm{b}} \\
\text { Members }\end{array}$ & $\begin{array}{l}\sim 30 \mathrm{Myr}^{\mathrm{c}} \\
\text { Foreground }\end{array}$ & $\begin{array}{c}\text { NM or Rejected } \\
\text { Sources }\end{array}$ & $\begin{array}{c}\text { All } \\
\text { Sources }\end{array}$ \\
\hline $\mathrm{C} 4$ & 4 & 3 & 7 & 23 & 131 & 161 \\
\hline C13 & 152 & 22 & 174 & 16 & 513 & 703 \\
\hline C4 AND C13 & 0 & 2 & 2 & 1 & 10 & 13 \\
\hline C4 OR C13 & 156 & 23 & 179 & 38 & 634 & 851 \\
\hline All & 156 & 23 & 179 & 38 & 634 & \\
\hline Periodic & $127(0.81)$ & $21(0.91)$ & $148(0.83)$ & $37(0.97)$ & $307(0.48)$ & \\
\hline Multi-periodic & $32(0.21)$ & $8(0.35)$ & $40(0.22)$ & $12(0.32)$ & $81(0.13)$ & \\
\hline No IR excess & $55(0.35)$ & $16(0.70)$ & $71(0.40)$ & $36(0.95)$ & $585(0.92)$ & \\
\hline High-conf IR excess & $94(0.60)$ & $7(0.30)$ & $101(0.56)$ & $1(0.03)$ & $35(0.06)$ & \\
\hline Possible IR excess & $7(0.04)$ & $0(0.00)$ & $7(0.04)$ & $1(0.03)$ & $14(0.02)$ & \\
\hline IR excess + periodic & $67(0.43)$ & $5(0.22)$ & $72(0.40)$ & $1(0.03)$ & $8(0.01)$ & \\
\hline IR excess + multi-periodic & $11(0.07)$ & $2(0.09)$ & $13(0.07)$ & $0(0.00)$ & $2(0.00)$ & \\
\hline Burster & $22(0.14)$ & $3(0.13)$ & $25(0.14)$ & $0(0.00)$ & $0(0.00)$ & \\
\hline Burster+IR excess & $22(0.14)$ & $3(0.13)$ & $25(0.14)$ & $0(0.00)$ & $0(0.00)$ & \\
\hline Burster + IR excess + periodic & $14(0.09)$ & $2(0.09)$ & $16(0.09)$ & $0(0.00)$ & $0(0.00)$ & \\
\hline Dipper & $18(0.12)$ & $3(0.13)$ & $21(0.12)$ & $0(0.00)$ & $0(0.00)$ & \\
\hline Dipper + IR excess & $18(0.12)$ & $3(0.13)$ & $21(0.12)$ & $0(0.00)$ & $0(0.00)$ & \\
\hline Dipper + IR excess + periodic & $16(0.10)$ & $2(0.09)$ & $18(0.10)$ & $0(0.00)$ & $0(0.00)$ & \\
\hline Spectral type & $151(0.97)$ & $22(0.96)$ & $173(0.97)$ & $26(0.68)$ & $155(0.24)$ & \\
\hline Gaia parallax & $129(0.83)$ & $20(0.87)$ & $149(0.83)$ & $38(1.00)$ & $558(0.88)$ & \\
\hline IRAC-1 & $135(0.87)$ & $4(0.17)$ & $139(0.78)$ & $6(0.16)$ & $168(0.26)$ & \\
\hline WISE-1 & $156(1.00)$ & $23(1.00)$ & $179(1.00)$ & $38(1.00)$ & $633(1.00)$ & \\
\hline AKARI 18 & $39(0.25)$ & $2(0.09)$ & $41(0.23)$ & $0(0.00)$ & $14(0.02)$ & \\
\hline MIPS-24 & $98(0.63)$ & $9(0.39)$ & $107(0.60)$ & $12(0.32)$ & $85(0.13)$ & \\
\hline MIPS-70 & $40(0.26)$ & $2(0.09)$ & $42(0.23)$ & $0(0.00)$ & $4(0.01)$ & \\
\hline PACS 70 & $64(0.41)$ & $2(0.09)$ & $66(0.37)$ & $0(0.00)$ & $4(0.01)$ & \\
\hline$V$ and $K_{\mathrm{s}}$ measured & $57(0.37)$ & $17(0.74)$ & $74(0.41)$ & $22(0.58)$ & $207(0.33)$ & \\
\hline$V$ from APASS & $19(0.12)$ & $4(0.17)$ & $23(0.13)$ & $8(0.21)$ & $262(0.41)$ & \\
\hline$\left(V-K_{\mathrm{s}}\right)$ via Gaia $G-K_{\mathrm{s}}$ & $36(0.23)$ & $2(0.09)$ & $38(0.21)$ & $5(0.13)$ & $145(0.23)$ & \\
\hline$\left(V-K_{\mathrm{s}}\right)$ via PanSTARRS $g-K_{\mathrm{s}}$ & $34(0.22)$ & $0(0.00)$ & $34(0.19)$ & $2(0.05)$ & $13(0.02)$ & \\
\hline SED-interpolated $V$ & $10(0.06)$ & $0(0.00)$ & $10(0.06)$ & $1(0.03)$ & $7(0.01)$ & \\
\hline$A_{v}$ from $J H K_{\mathrm{s}}$ diagram & $31(0.20)$ & $4(0.17)$ & $35(0.20)$ & $9(0.24)$ & $313(0.49)$ & \\
\hline$A_{v}$ from spectral type & $115(0.74)$ & $15(0.65)$ & $130(0.73)$ & $16(0.42)$ & $107(0.17)$ & \\
\hline$A_{v}$ from SED fits & $5(0.03)$ & $4(0.17)$ & $9(0.05)$ & $11(0.29)$ & $25(0.04)$ & \\
\hline Median $A_{v}$ assigned & $5(0.03)$ & $0(0.00)$ & $5(0.03)$ & $2(0.05)$ & $189(0.30)$ & \\
\hline
\end{tabular}

Notes.

${ }^{a}$ Numbers in the table are raw number of stars meeting the stated criterion/criteria, followed by the sample fraction in parentheses.

b $\sim 3$ Myr members are the high-confidence (Taurus) member plus the possible (Taurus) member sample.

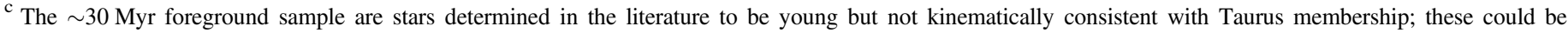
members of, e.g., Group 29 (see Section 2.2).

comparison of our periods to those from the literature. This section ends with color-magnitude diagrams (CMD) for the sample. Section 4 discusses the influence of disks on the period distribution. Section 5 presents the distributions of periods and periods against color as a proxy for mass. We also compare Taurus to the rest of the clusters we have analyzed with K2 data (Papers I-V). In Section 6, we characterize the LCs in the same fashion as we did for the other clusters. Finally, we summarize our results in Section 7.

We note explicitly that there are four sets of stars discussed in this paper: (1) highest-quality Taurus members, (2) lowerquality members (in other words, possible Taurus members), (3) a population largely foreground to Taurus that is likely older than Taurus but still young $(\sim 30 \mathrm{Myr})$, and (4) nonmembers (NMs). Appendix A contains a detailed description of how we define these categories, with an overview in Section 2.2. When we use the term "members", we are referring to the highest-quality plus the possible Taurus members. When we use the term "foreground" (as in "foreground population"), we are referring to the set of stars that are older than Taurus but still relatively young.

\section{Data \\ 2.1. K2 Data}

Stars in the Taurus Molecular Cloud were observed in two different K2 campaigns, Campaign 4 (C4; 2015 February 7-2015 April 24) and Campaign 13 (C13; 2017 March 8-2017 May 27), with the majority of Taurus members coming from C13; see Figure 1. As in our earlier papers, we start by considering all possible cluster members and then narrow the sample to high-confidence and lower-confidence members (see Section 2.2); results for the likely NMs are listed in the Appendix for reference. There are $\sim 850$ candidate Taurus 


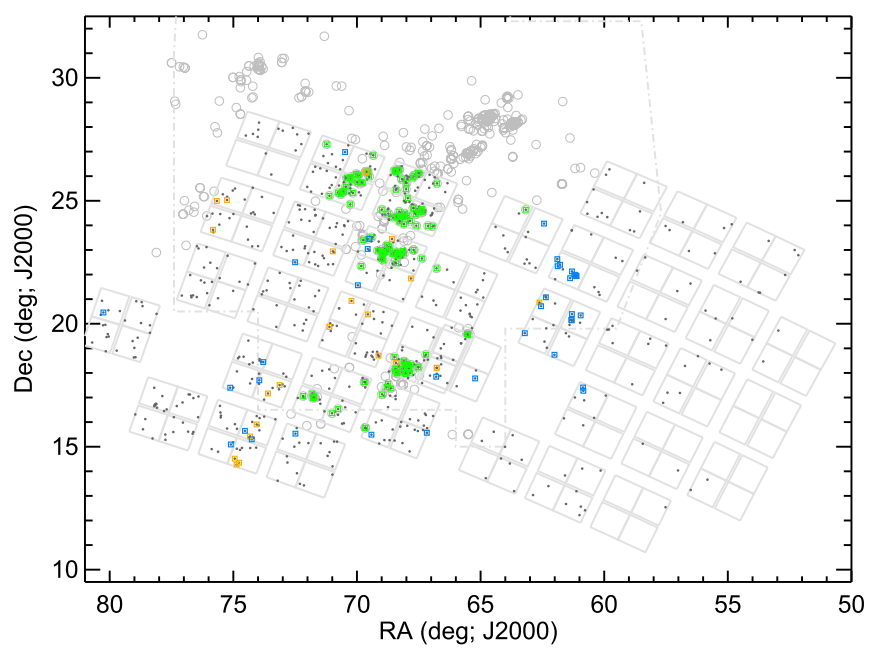

Figure 1. Locations of targets of note projected onto the sky. Small dark gray circles: candidate Taurus members with LCs. Additional green square: highestquality Taurus members (see Section 2.2 for more on membership). Additional orange: possible Taurus members. Additional blue square: foreground population, e.g., stars determined in the literature to be young but not kinematically consistent with Taurus membership; these could be members of, e.g., Group 29 (see Section 2.2). For context, the large light gray squares are the $\mathrm{K} 2$ footprints ( $\mathrm{C} 13$, left; $\mathrm{C} 4$, right), and the small light gray circles are the Taurus members as compiled by Esplin \& Luhman (2019). The dashed-dotted line is the polygon considered for potential Taurus members using WISE in Rebull et al. (2011). The K2 observations cover about $30 \%$ of the likely Taurus members from Esplin \& Luhman (2019).

members with K2 LCs from either campaign and a few with K2 data from both campaigns (see Table 1). All of the LCs used here were observed in the long-cadence $(\sim 30$ minutes cadence) mode.

Our analysis (see Section 2.2 below) focused on the 156 highest-quality Taurus members, 23 possible members, and 38 foreground objects that are young (but likely older than the Taurus molecular cloud population). Figure 1 shows the distribution of these objects with $\mathrm{K} 2$ LCs on the sky, in context with other reference points. The area defined as encompassing "Taurus" by, e.g., Esplin \& Luhman (2019) goes further north (and east) than the $\mathrm{K} 2$ region, such that the $\mathrm{K} 2$ observations cover about $30 \%$ of the members from Esplin \& Luhman (2019). Assuming that there should be no spatial dependence of stellar rotation rate on location in the cluster, there should be no bias in our distribution of periods resulting from the incomplete coverage of the cluster. However, specifically because the $\mathrm{K} 2$ region extends over a larger area to the south and west, it encompasses a larger area than has been studied most intensively for potential Taurus members, and enables LC collection from a more dispersed population of candidate young stars.

There are very few stars considered to be Taurus members of any confidence level that have LCs in both the C4 and C13 campaigns. Of these, just two are in the set of possible Taurus members (EPIC $210689309=\mathrm{AG}+18339$; EPIC $210689130=$ $\mathrm{HD}$ 28150), and one is in the foreground population (210662824 $=$ HD 285778). All three of these are periodic in both campaigns. There are an additional 10 field stars with two sets of LCs, and four of them have periods in both campaigns. For all stars in common between C4 and C13 (whether or not they are members), the periods derived separately from both campaigns are the same within $\sim 4 \%$ accuracy, suggesting that the effective astrophysical uncertainty on the periods is likely very low, at least for stars without disks.

Our analysis follows the same approach discussed in our earlier papers (Papers I-V) and is briefly summarized here. For each target, we selected the best (see Paper I) LC from several different available LC versions: (1) the pre-search data conditioning (PDC) version generated by the Kepler project ${ }^{7}$ and obtained from the Mikulski Archive for Space Telescopes (MAST); (2) a version using custom software developed by coauthor A.M.C.; (3) the "self-flat-fielding" approach used by Vanderburg \& Johnson (2014) and the K2SFF pipeline as obtained from MAST; (4) the LCs from the EVEREST2 pipeline (Luger et al. 2016, 2018), which uses pixel-level decorrelation, as obtained from MAST; and (5) the EVEREST2 pipeline LCs, where large-scale structure is removed via a least-squares (Savitzky-Golay) polynomial smoothing filter (e.g., Press et al. 1992), as implemented in the IDLastro library. ${ }^{8}$ We removed any data points corresponding to thruster firings and any others with bad data flags set in the corresponding data product. Any periodic signals are generally unambiguous and are generally detected in all of the LC versions. The spacecraft slowly drifts and then repositions regularly every 0.245 day, so we particularly scrutinized any detected periods near 0.245 day.

$\mathrm{K} 2$ has a relatively large pixel size $(3 !$ ! $98 \times 3$ !"98). To identify sources subject to confusion, we inspected the region around each target with the Finder Chart tool $^{9}$ from NASA's Infrared Science Archive (IRSA). We also incorporated the diagnostic information provided by the various $\mathrm{K} 2$ data reduction pipelines. For those targets where source confusion is a concern, we either took the existing LC most likely to be centered on the target given the diagnostic information provided by the corresponding pipelines or re-reduced the data using the Cody approach but with a small, fixed aperture.

We omitted two of the highest-quality members (see Section 2.2): EPICs 247031436 (HD 28867B; confused with nearby sources) and EPIC 247989931 (2MASS J04412464 +2543530; too faint). For completeness, we note that EPIC 211068851 (HD 26212) is too bright and EPIC 210893410 is too faint, but both are also unlikely to be Taurus or foreground members.

There are a few targets that are either erroneously listed as distinct targets in the EPIC catalog or could be a faint source next to a brighter source; in either case, a good LC cannot be obtained. HL Tau (EPIC 210690913) and XZ Tau AB (EPIC 210690892) are high-quality members and close together in the sky, though resolvable; the XZ Tau binary itself is not resolved. An additional target is listed as EPIC 210690886, but it is too faint and/or close to XZ Tau to measure independently. Given its location, position matching with literature data (see Section 2.3 below) accidentally picks up several matches that should have been matched to $\mathrm{XZ}$ Tau $\mathrm{AB}$. As a result, EPIC 210690886 is explicitly removed from our database as effectively a duplicate. DR Tau (EPIC 246923113) is also a high-quality member. EPIC 246923117 is 0 ". 22 away from DR Tau and is explicitly removed as effectively a duplicate. EPIC 246926943 is a real though optically faint target near a brighter source. The LC is not easily distinguishable from that of its bright neighbor. This target is listed as an NM as a result.

\footnotetext{
7 https://keplerscience.arc.nasa.gov/k2-pipeline-release-notes.html

8 https://idlastro.gsfc.nasa.gov

9 http://irsa.ipac.caltech.edu/applications/finderchart
} 


\subsection{Membership}

\subsubsection{Summary}

The details of our membership selection process are given in Appendix A. To summarize, we have selected 156 of the "highest-confidence" Taurus members, 23 "possible" Taurus members, and 38 stars that constitute an older ( $30 \mathrm{Myr})$ "foreground" population. The highest-confidence members are those objects listed as Taurus members in Esplin \& Luhman (2019). The possible members are additional objects selected individually by us from the available literature and Gaia DR2 kinematic data as Taurus members; these are generally more dispersed than the highest-confidence members. The $\sim 30 \mathrm{Myr}$ foreground population includes (but is not limited to) members of Group 29 (Oh et al. 2018; Luhman 2018); some stars might potentially belong to $\mathrm{Mu}$ Tau (M. Gagne et al. 2020, in preparation; Liu et al. 2020) or possibly Cas-Tau (e.g., Hartmann et al. 1991; de Zeeuw et al. 1999; Luhman 2018; also see the appendix of David et al. 2018 and references therein).

Membership status is used in Table 1 for the sample breakdown and is included in Table 2. The highest-confidence sample is listed as distinct from the possible member sample. The highest-confidence and possible member samples are combined into one sample listed as the $\sim 3 \mathrm{Myr}$ member sample. The foreground population is a distinct sample with different kinematics and mean distance, and it is likely significantly older than the Taurus population. For completeness, Table 1 also includes the NM sample. Appendix A.2 lists individually the information on stars in the probable NMs.

Unless explicitly indicated, the subsequent analysis in this paper uses the 156 most confident members (typically colored green in the figures) plus the 23 possible members (typically colored yellow-orange in the figures) as the member sample. The 38 stars in the foreground older population are colored blue in the figures.

\subsubsection{Sample Statistics}

In general, we expect that Taurus members (in comparison to the NMs) will have a higher fraction of disk indicators, a Gaia DR2 parallax between about 6 and 8 mas, and a high fraction of periodic LCs. In practice, since efforts to determine spectral types have focused on members, we also expect members to have a higher fraction of spectral types in the literature. As can be seen in Table 1, all of this can be found in the Taurus member sample. There is a high fraction of spectral types (Section 2.3), periodic stars (Section 3.1), long-wavelength IR detections, and, therefore, disked stars (Section 2.4) among the members.

The foreground sample, because it is older ( $\sim 30 \mathrm{Myr}$ rather than $\sim 3$ ), has a lower fraction of disks (and IR detections). It also has a higher fraction of periodic stars, but this is highly biased, since we started from the set of K2 LCs in C4/C13, not from the set of all possible members of this foreground population (which may extend over more K2 campaigns).

The reddening as determined above (Section 2.5) shows that for the highest-confidence members, the distribution of $A_{K}$ peaks at $\sim 0.1$ with a tail to $\sim 2$; both the groups of possible members and the foreground have $A_{K}<0.3$, with a peak $<0.05$.

Among our K2 work in older clusters, a very high fraction of the members are periodic-92\% in Pleiades (Papers I-II), 87\% in Praesepe (Paper IV), and $86 \%$ in USco (Paper V). We theorized that the lower fractions in Praesepe and USco may be indicative of NM contamination. In Taurus, $81 \%$ of the highest-quality members stars are periodic; in $\rho \mathrm{Oph}$, only $61 \%$ are periodic. Two things are likely contributing to the lower fraction of periodic stars in these youngest clusters. Disk emission affects period determination in that stochastic contributions from the disk make periods harder to find. Additionally, NM contamination is also likely to be a factor. Far more effort has been devoted to determining Taurus membership than membership for $\rho$ Oph, so it is more likely that there is a higher NM contamination rate in $\rho$ Oph. The disk fraction among $\rho$ Oph member stars with a K2 LC is $46 \%$, very similar to the Taurus member sample of $\sim 56 \%$ (Table 1 ); $\rho$ Oph is thought to be younger ( $\sim 1$ Myr) than Taurus ( $\sim 3$ Myr) and, thus, should have more disks. In both cases, most of the disked stars are also periodic. We suspect that the NM disk-free contamination is higher in $\rho$ Oph than Taurus, at least partially accounting for the relatively low fraction of periodic stars in $\rho$ Oph. In contrast, the membership is likely better in Taurus, and the lower fraction of periodic stars arises primarily from "pollution" of the LC by disk-related effects, making periods harder to find.

We note as well that the lowest-mass Taurus members studied here extend into the brown dwarf regime (see, e.g., Scholz et al. 2018). These mid-M stars represent the youngest, lowest-mass stars with high-quality LCs yet observed.

\subsection{Literature Data}

The literature on stars in (or around or near) Taurus is rich indeed. Aiming for uniform consistency rather than completeness, rather than scouring the literature for individual photometric measurements, we assembled information on each target from several all-sky or large-scale surveys. We matched by position to each catalog with typical matched positions within an arcsecond.

The optical data came from several catalogs. We searched in Gaia DR1 (Gaia Collaboration et al. 2016) for the $G$ magnitude consistent with our prior analyses, and DR2 (Gaia Collaboration et al. 2018) for the more recent $G, R_{p}$, and $B_{p}$ photometry, in addition to parallaxes and proper motions. The kinematic data provided by Gaia DR2 are particularly useful in identifying members (Section 2.2 includes the Gaia DR2 ID; see Table 1 for fractions of samples with Gaia parallaxes). The distances we used here are those provided by Bailer-Jones et al. (2018). Additional optical data were obtained from the AAVSO Photometric All-Sky Survey (APASS; Henden et al. 2016), specifically, $V$ magnitudes. We used both Pan-STARRS1 (Chambers et al. 2016) and the Sloan Digital Sky Survey (e.g., Ahn et al. 2012) for multiband optical data. Every star here has several optical measurements.

We used infrared data from three all-sky surveys: the TwoMicron All Sky Survey (2MASS; Skrutskie et al. 2006) at $J, H$, and $K_{\mathrm{s}}$; the Widefield Infrared Survey Explorer (WISE; Wright et al. 2010) at 3.5, 4.6, 12, and $22 \mu \mathrm{m}$; and AKARI (Murakami et al. 2007) data at 9, 18, 65, 90, 140, and $160 \mu \mathrm{m}$. WISE and AKARI have some similar wavelengths, but they have very different sensitivities. We also used infrared data from two pointed missions (as opposed to all-sky surveys). For the Spitzer Space Telescope (Werner et al. 2004), we started with data from the Spitzer Enhanced Imaging Products (SEIP) ${ }^{10}$ at $3.6,4.5,5.8,8$, and $24 \mu \mathrm{m}$, which aggregates all data from the cryogenic portion of the Spitzer mission. The SEIP only

\footnotetext{
${ }^{10}$ http://irsa.ipac.caltech.edu/data/SPITZER/Enhanced/SEIP/overview.html
} 
Table 2

Contents of Table: Periods and Supporting Data for Taurus Members with K2 Light Curves

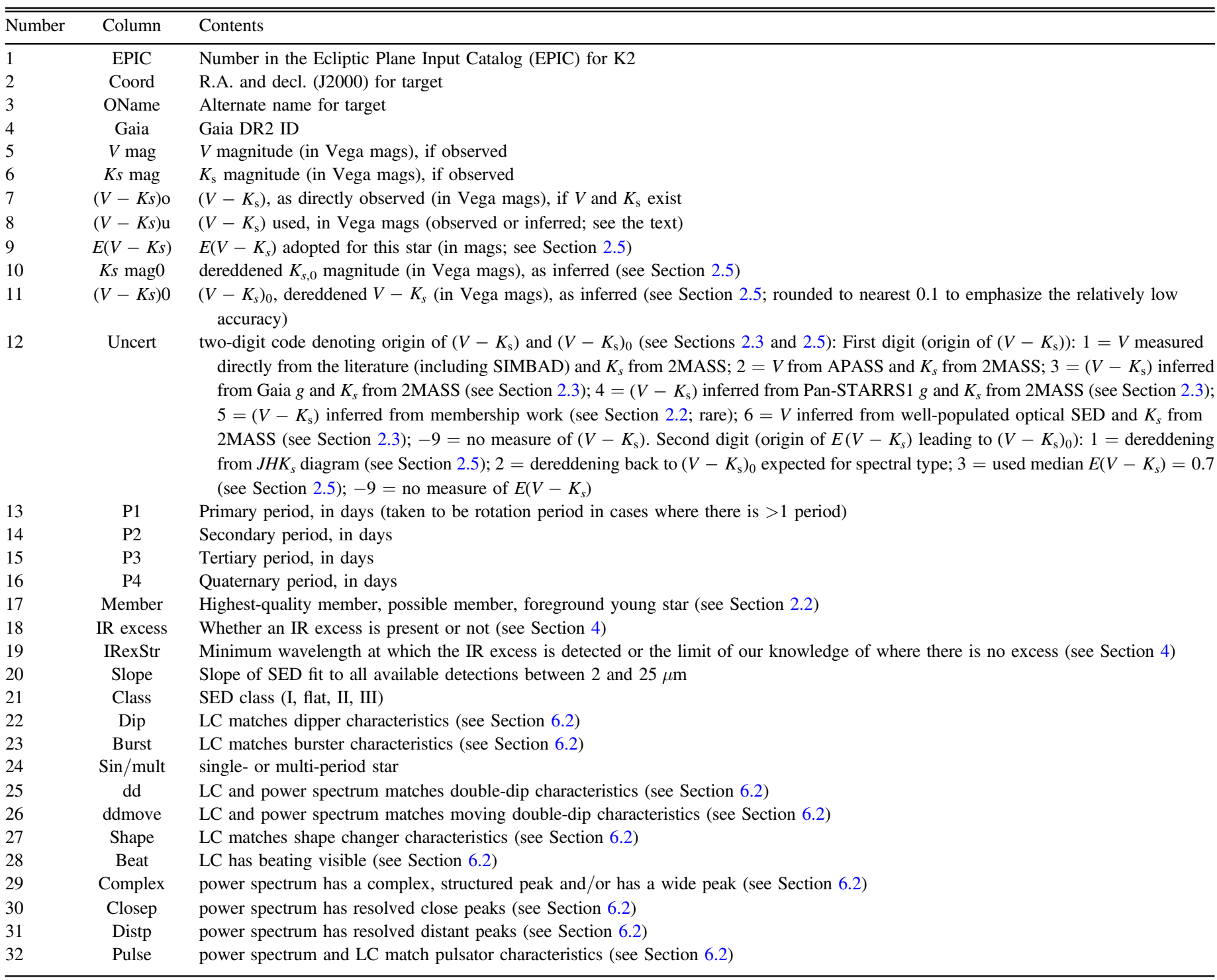

(This table is available in its entirety in machine-readable form.)

includes objects with high signal-to-noise ratio detections; when flux densities did not appear in the SEIP for our targets, we used catalog data from the Spitzer Taurus project (Rebull et al. 2010) for any bands between 3.6 and $70 \mu \mathrm{m}$ (note that $70 \mu \mathrm{m}$ was not included in the SEIP, so all $70 \mu \mathrm{m}$ data come from the Spitzer Taurus project). Most of the members have a Spitzer counterpart; a relatively low fraction of the NMs have a Spitzer counterpart (see Table 1). Relatively few stars in the sample have a Spitzer/MIPS counterpart at 24 or $70 \mu \mathrm{m}$. Herschel data cover a portion of the Taurus region. Analogous to the SEIP, the Herschel Space Observatory (Pilbratt et al. 2010) Highly Processed Data Products for PACS 70, 100, and $160 \mu \mathrm{m}$ (Marton et al. 2017) incorporate all of the data from the mission. Very few targets have a PACS counterpart, but a higher fraction of the members are detected (a rate that is biased by the observations that targeted known members). K2 generally monitored optically bright stars, thereby selecting against embedded stars bright in the mid- and far-infrared.
In general, there is a steep drop off in counterpart numbers as wavelength increases. Table 1 includes the numbers and fractions of stars in several membership categories (Section 2.2) for several representative infrared bands. However, the fraction of Taurus member stars (not foreground, not NM) that are detected out to long wavelengths is much higher than the fraction of NM stars, as expected-Taurus member stars are more likely to have an IR excess and, thus, be detected in these various infrared data sets.

We used all of these photometric data to assemble a spectral energy distribution (SED) for each target. If the data from one catalog were obviously inconsistent with the rest of the SED, then we removed the data points from that catalog for that source on the assumption that the positional match failed. We also used the IRSA Finder Chart tool (as well as its sister tool, IRSA Viewer) to investigate source mismatches.

Our earlier papers use $\left(V-K_{\mathrm{s}}\right)_{0}$ as a proxy for mass, so to better enable inter-cluster comparisons, we continue to do that here. All of the stars have measured $K_{\mathrm{s}}$, leaving us to find or 
calculate $V$. A substantial fraction of the targets have measured $V$ magnitudes that we could assemble from the literature above, but several stars still lack $V$ measurements. If a Gaia $G$ magnitude is available (from DR1), then $\left(V-K_{s}\right)$ was interpolated from $\left(G-K_{s}\right)$ as in Paper IV (which used DR1 Gaia data to establish this relationship); we estimate errors on these estimates to be $\sim 0.017-0.085$ mag. For stars redder than $\left(V-K_{s}\right) \sim 5$, the relation from Paper IV is linearly extrapolated to $\left(V-K_{s}\right) \sim 8$. Similarly, if no Gaia $G$ mag is available, but a Pan-STARRS1 $g$ is available, then $\left(V-K_{s}\right)$ can be calibrated via an empirical relation between $\left(g-K_{s}\right)$ and $\left(V-K_{\mathrm{s}}\right)$; errors on these estimates are probably comparable to those from Gaia-derived colors. As a last resort, for those stars still missing a $V$ estimate, since the SED is well populated in the optical using literature photometry, a $V$ magnitude is linearly interpolated from the SED. All of the targets thus have a measured or inferred $\left(V-K_{\mathrm{s}}\right)$.

Table 2 includes, for the most likely members, possible members, and foreground population (identified in Section 2.2), the relevant supporting photometric data, including the observed or interpolated $\left(V-K_{\mathrm{s}}\right)$, plus the periods we derive (in Section 3.1) and the IR excess assessments (Section 2.4). A similar table with all of the likely NMs appears in Appendix A.2.

Most of the Taurus members have spectral types in the literature (Table 1). We assembled spectral types largely from Luhman et al. (2017), Luhman (2018), and Esplin \& Luhman (2017, 2019). Some individual sources have spectral types not collected in these papers, and we assigned those spectral types individually based on the literature.

For those stars with spectral types, we used the spectral types as part of the process for identifying disks (see Section 2.4) and determining the best value of reddening (see Section 2.5). For those stars with spectral types, we used a Kurucz-Lejeune (Kurucz 1993; Lejeune et al. 1997) model corresponding to that spectral type and normalized to the data at the $J$ band. We extended a Rayleigh-Jeans line from the longest wavelength model data point to beyond $24 \mu \mathrm{m}$. Using the reddening law from Mathis (1990), we reddened the models for a grid of $A_{J}$ values, calculated a reduced chi-sq $\left(\chi_{\nu}^{2}\right)$ comparing the model to the data at all optical bands ( $U$, if available) through $J$. We selected the best fit based on the smallest $\chi_{\nu}^{2}$. These model fits to the SED are not meant to be rigorous but to instead "guide the eye."

\subsection{Disk Indicators}

A substantial fraction of the Taurus members have an IR excess, from which we can infer the presence of a circumstellar disk (see summary statistics in Table 1). In the context of this paper (as for Paper V), we wish to have a complete list of disks, as opposed to an unbiased list; we identify a star as a disk candidate if it has a plausibly real excess at any IR wavelength (at which we have a detection) in the catalog we assembled. The wavelength at which the IR excess begins is included in Table 2 (and in the Appendix in Table B1 for the NMs).

Because WISE has a lower spatial resolution than Spitzer, we used IRSA's Finder Chart tool to inspect the WISE images to see if the detections in the catalog reflect what can be seen in the images. Again following Paper V, to identify disks, we looked at the significance of any putative IR excess at 12, 22, and $24 \mu \mathrm{m}$ where available (with $\chi$ calculated as described in Paper V), taking into account that for the latest types, the expected photospheric colors may be $>0$. We used the empirical photospheric infrared color [W1]-[W3] as a function of $\left(V-K_{\mathrm{s}}\right)_{0}$ from Paper V. We then assessed the ensemble of information available for all sources (e.g., all points $\geqslant 2 \mu \mathrm{m}$, the shape of SED, the results of the simple model fit discussed in Section 2.3 above, etc.). For each source, we have an assessment of whether it has a disk, and if so, the shortest wavelength likely contributing to an IR excess.

In this fashion, as for Paper $\mathrm{V}$, we identified unambiguous disk candidates and non-disk candidates (at least, non-disks given the available data, which often extend at least to 12 or $22 \mu \mathrm{m}$ ), with a few percent of ambiguous (possible) disks noted as such. Table 1 includes the number and sample fraction for each of the relevant samples. More than half of the Taurus members have disks; there is just one high-confidence (and one more possible) disk candidate among the foreground ( $\sim 30 \mathrm{Myr}$ ) population. Note that the disk excess criteria are conservative and that the non-disked sample will likely have contamination from weaker $(<5-10 \sigma$ excess $)$ disks. Note also that the lowest-mass bin is likely incomplete in the non-disks due to sensitivity issues (stars with excesses are more likely to be detected at long wavelengths than stars without excesses). While this sample draws from many surveys and wavelengths, in order to be considered at all, there must be an observation in $\mathrm{K} 2$, which requires targeting of the source by a human, and therefore, the sample is affected not only by extinction but pixel mask selection.

Independent of the disk candidate status, we performed a simple ordinary least-squares linear fit to all available photometry (with errors, but just detections, not including upper or lower limits) between 2 and $24 \mu \mathrm{m}$, inclusive. In the spirit of Wilking et al. (2001), we define $\alpha=d \log \lambda F_{\lambda} / d \log \lambda$, where $\alpha>0.3$ for a Class I, 0.3 to -0.3 for a flat-spectrum source, -0.3 to -1.6 for a Class II, and $<-1.6$ for a Class III. The slope and the class are both available in Table 2 (and Table B1, where it is assumed that the IR excess is due to a circumstellar disk, which may not be a good assumption).

For completeness, we note two items. First, Table 1 indicates that there are NM with IR excesses. These objects include evolved stars with dusty winds or envelopes, distant Be stars, and a few extragalactic objects (active galactic nuclei, quasistellar objects). Second, Tables 2 and B1 indicate the onset of IR excess for each target. Since the IR excess could affect the $K_{\mathrm{s}}$ bands, which could cause an overestimation of $A_{V}$ in the methods described below, we note that such targets are a minority here; $>80 \%$ of the highest-quality members have disks that start at longer than $3 \mu \mathrm{m}$.

\subsection{Dereddening}

The reddening in the direction of Taurus can be substantial and patchy. As noted above, we obtained spectral types from the literature. In order to deredden the $V-K_{s}$ colors, we followed the same approach as in Paper V (specifically for $\rho$ Oph), with some modifications.

We can place essentially all of the stars on a $J-H$ versus $H-K_{s}$ diagram. We can deproject much of the sample back along the reddening law derived by Indebetouw et al. (2005) to the expected $J H K_{s}$ colors for young stars from Pecaut \& Mamajek (2013) or the T Tauri locus from Meyer et al. (1997). Note that there is a discontinuity between the end of the Pecaut \& Mamajek relation and the beginning of the T Tauri locus (noted in Meyer et al. 1997 and Paper V); this results in a small gap in the dereddened $\left(J-K_{s}\right)_{0}$ distribution between $\sim 0.9$ and 
$\sim 1.0$. The reddening so derived can be converted to $E\left(V-K_{s}\right)$ via $A_{K}=0.114 A_{V}$ (Cardelli et al. 1989). This approach worked well for Upper Sco in Paper V, but it did not work as well in $\rho$ Oph, primarily because there is significantly higher reddening. Similarly, there is high reddening toward some of the stars in Taurus, and there is a lot of scatter, even in the NIR colors.

Another approach used in Paper V (and preferred in $\rho$ Oph) was to take the spectral type, compare the observed colors to those expected for that type from Pecaut \& Mamajek (2013), and calculate the resultant reddening. Consequently, any distribution of colors for stars dereddened in this fashion will be quantized, because, e.g., all stars of type M1 are assigned to have the same color. Due to the high fraction of spectral types for Taurus members, we can calculate reddening following the above prescription for most of the members.

Because there is patchy reddening and a lot of scatter in the $J H K_{\mathrm{s}}$ diagram (much more than for USco, for example), we took the reddening estimate from the spectral type first. If that value was unphysical (e.g., $<0$ ), then we took the value derived from the $J H K_{\mathrm{s}}$ diagram. Some of those values were still unphysical or insufficient (e.g., $\left(V-K_{\mathrm{s}}\right)_{0}$ was still $>8$ or 10$)$. So, for those stars with spectral types, we used the KuruczLejeune model grid fitting described above (Section 2.3) to estimate the reddening in a third way. For several of the foreground population lacking spectral types, the reddening derived from the $J H K_{\mathrm{s}}$ diagram was substantially discrepant from the SED fitting having guessed a spectral type based on the observed $\left(V-K_{\mathrm{s}}\right)$. In those cases, we selected the best reddening from the SED fitting. As a fourth and last resort, we assigned a star the modal reddening of $E\left(V-K_{s}\right) \sim 0.46$ determined via the comparison of observed values to that expected from the spectral type for the ensemble.

Table 1 includes the numbers and sample fractions for each of these approaches. The value used for most stars comes from either of the first two approaches (spectral type or $J H K_{\mathrm{s}}$ diagram). For relatively few stars do we have to fall back to the most likely reddening.

The dereddened $\left(V-K_{\mathrm{s}}\right)_{0}$ we used for each object is included in Table 2 for the members and in Appendix A.2 for the NMs. However, to emphasize the net uncertainty, the "vmk0" column in Table 2 has been rounded to the nearest $0.1 \mathrm{mag}$. The values used in the plots here can be recovered by using the $E\left(V-K_{s}\right)$ ("ev-k") and $\left(V-K_{s}\right)_{\text {observed ("vk-used") }}$ columns.

The net errors are hard to quantify after all of these steps. Table 2 (and its analogous Table B1 for NMs) include a twodigit code indicating the origin of the $\left(V-K_{\mathrm{s}}\right)$ value and the method by which the $\left(V-K_{\mathrm{s}}\right)$ was dereddened to $\left(V-K_{\mathrm{s}}\right)_{0}$ (see Table 2 or B1 for specific definitions). The reddening can be large and a significant source of uncertainty. Via internal comparisons and uncertainties not just on the assumed photospheric colors but also uncertainties in spectral typing, we estimate that the typical uncertainty for Taurus members could be as much as 1 magnitude in $E\left(V-K_{s}\right)$. To quantify this further, for the highest-quality members, we compared the reddening values derived from the $J H K_{\mathrm{s}}$ diagram, from spectral types, and from SED fitting. The reddening derived from the SED is well matched to that from the $J H K_{\mathrm{S}}$ diagram; that from the spectral type has systematic offsets with respect to the others in the sense that the reddening from the spectral types is lower on average but also negative (e.g., unphysical) far more often. In all cases, a Gaussian fit to the distribution of fractional differences suggests that a scatter of $\sim 0.5 \mathrm{mag}$ in $E\left(V-K_{S}\right)$ is typical. Most of the $J H K_{\mathrm{S}}$ measurements come from 2MASS, where the measurements should have been roughly simultaneous for the three bands, so intrinsic variability alone cannot account for the scatter. It is not the case that emission from the disk is affecting the $J H K_{\mathrm{s}}$, because the $\mathrm{K} 2$ sample is biased toward optical sources, and as noted above, most $(>80 \%)$ of these disks become apparent at $3.5 \mu \mathrm{m}$ or longer. The large uncertainty in reddening is real but evidently unavoidable.

\section{Periods and CMDs}

\subsection{Finding Periods in the K2 LCs}

Our approach for finding periods was identical to that used in Papers I, II, IV, and V. In summary, we used the Lomb-Scargle (Scargle 1982) approach as implemented by the NASA Exoplanet Archive Periodogram Service ${ }^{11}$ (Akeson et al. 2013). We also used the IRSA Time Series Tool ${ }^{12}$, which employs the same underlying code as the Exoplanet Archive service but allows for interactive period selection. We looked for periods between 0.05 and 35 days, with the upper limit being set by roughly half the campaign length. Because the periods are typically unambiguous, false alarm probability (FAP) levels are calculated as exactly zero for most of the periods we present here (and the remaining FAP levels are typically $<10^{-4}$ ).

The periods we derive appear in Table 2 for all members and in Appendix A.2 for the NMs.

\subsection{Interpretation of Periods}

In Papers I, II, and IV, we described in detail the LC and periodogram shapes we observed in the older Pleiades and Praesepe clusters; Paper V included an additional section on the physical interpretation of the LCs of younger stars in USco and $\rho$ Oph. Here, we briefly summarize and refer the reader to those earlier papers for more detail.

Including both $\mathrm{C} 4$ and $\mathrm{C} 13,81 \%$ (127 stars) of the highestconfidence Taurus member sample stars have at least one identifiable period in their LC. We retain up to four periods; 32 $(21 \%)$ have more than one period. Nearly all of the foreground population has at least one period. More than $95 \%$ of the sample we focus on here (members of Taurus or belonging to the foreground) have LCs consistent with a surface spot origin. For stars with two (or more) periods that we believe are due to rotation, we plot only one point at the period we believe corresponds to the actual rotation period of the star dominating the $\left(V-K_{\mathrm{s}}\right)_{0}$ measurement. In those cases, particularly among the $\mathrm{M}$ stars, where there are two (or more) periods that are not close together, we believe the star to be a likely binary (or other multiple); see Papers II and IV, and Stauffer et al. (2018a).

In a few stars, just two of the possible members, there is a forest of very short period peaks in the periodogram. We take these to be pulsators.

There are seven stars in the entire sample that have little or no IR excess but have periodic LC shapes with scallop shell or flux dip morphologies, which cannot be due to spots or pulsation; see Stauffer et al. (2018b, 2017) for a discussion of these types of LC morphologies. Of these stars, three of

\footnotetext{
${ }^{11}$ http://exoplanetarchive.ipac.caltech.edu/cgi-bin/Periodogram/nphsimpleupload

12 http://irsa.ipac.caltech.edu/irsaviewer/timeseries
} 

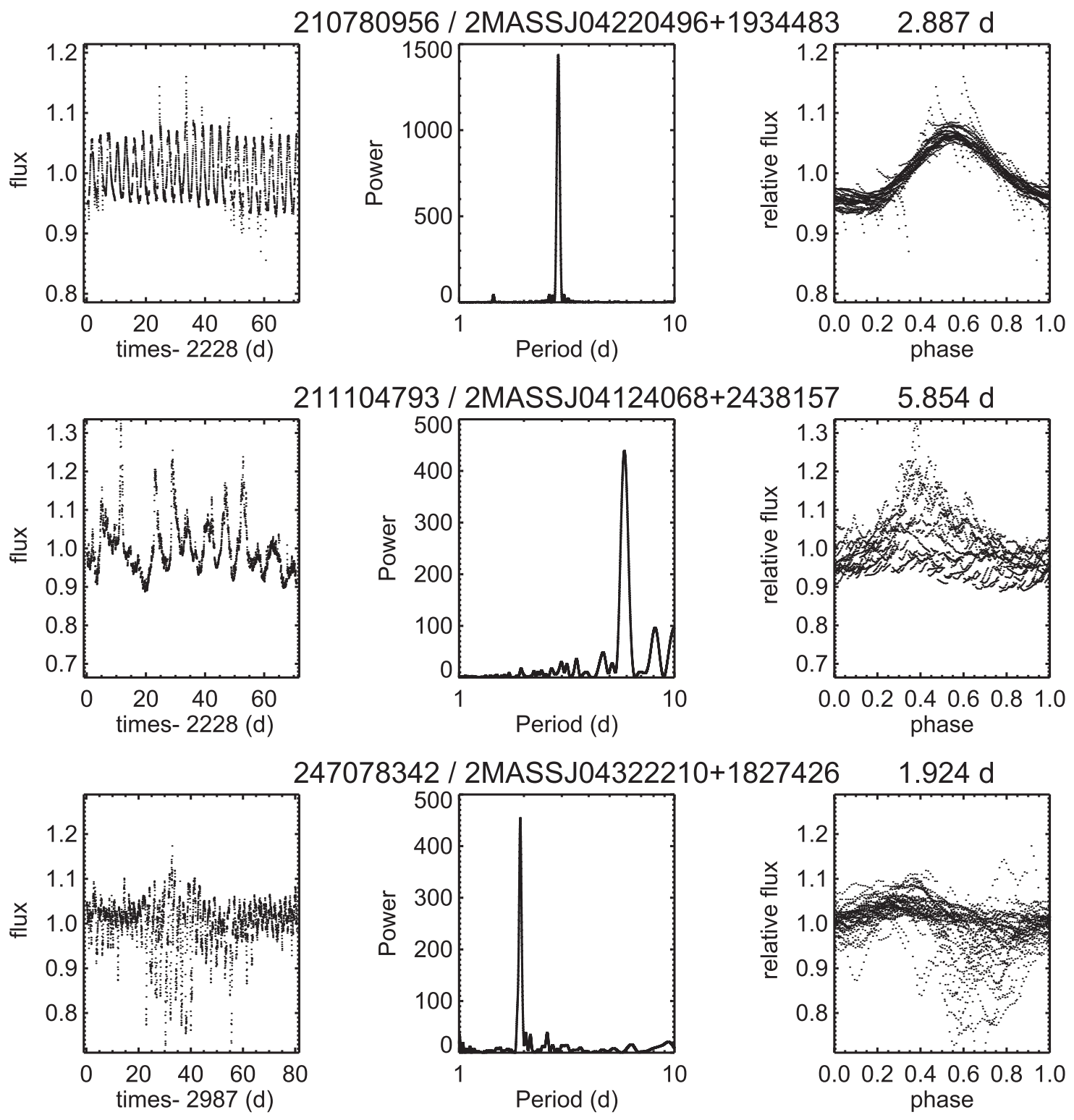

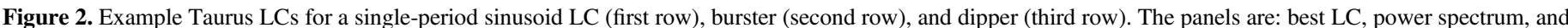

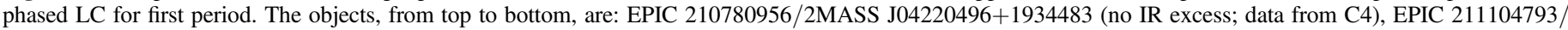

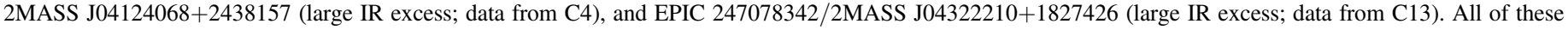
targets are confident members of Taurus.

them are highest-quality members (EPIC 246938594, EPIC $246969828=\mathrm{Cl}^{*}$ Melotte25LH19, and EPIC $247794636=$ 2MASS J04321786+2422149), three are possible members (EPIC $246676629=$ UCAC 4522-000989, $246682490=$ UCAC $\quad 4522-009859, \quad 247343526=2$ MASS J04405340 +2055471 ), and one is part of the foreground population (EPIC 246776923). Because the primary period in these cases is likely to either be a rotation period or strongly related to a rotation period, these were retained as rotation periods.

As in other young clusters, there are LCs with dipper or burster morphologies (see, Figure 2 and, e.g., Paper V; Cody et al. 2014; Stauffer et al. 2014, 2015, 2016a; Cody \& Hillenbrand 2018). All of the LCs we identified as dippers (21 members) or bursters (25 members) also have disks, and all are members of some sort (Table 1). These are identified in the corresponding tables (Table 2 and Appendix A.2). Note that the period we report as the rotation period is often but not always also the period of the dips/bursts; sometimes the dips/bursts align with the sinusoidal modulation, and sometimes they do not. N. Roggero et al. (2020, in preparation) will explore the dipper population in Taurus.

We categorize "timescales" for LCs that seem to have a repeating pattern, but the pattern does not seem to be due to starspots or other rotation-related phenomena and, therefore, are not taken as periodic; see Appendix B.

\subsection{Comparison to Literature Periods}

A detailed comparison between periods in the literature (consisting of a few to tens of stars per study) and periods we derive from $\mathrm{K} 2$ data is given in Appendix $\mathrm{C}$.

In summary, we conclude that we are recovering most of the periods reported in the literature. The results are a mix of excellent agreement, likely harmonics reported in the literature, 


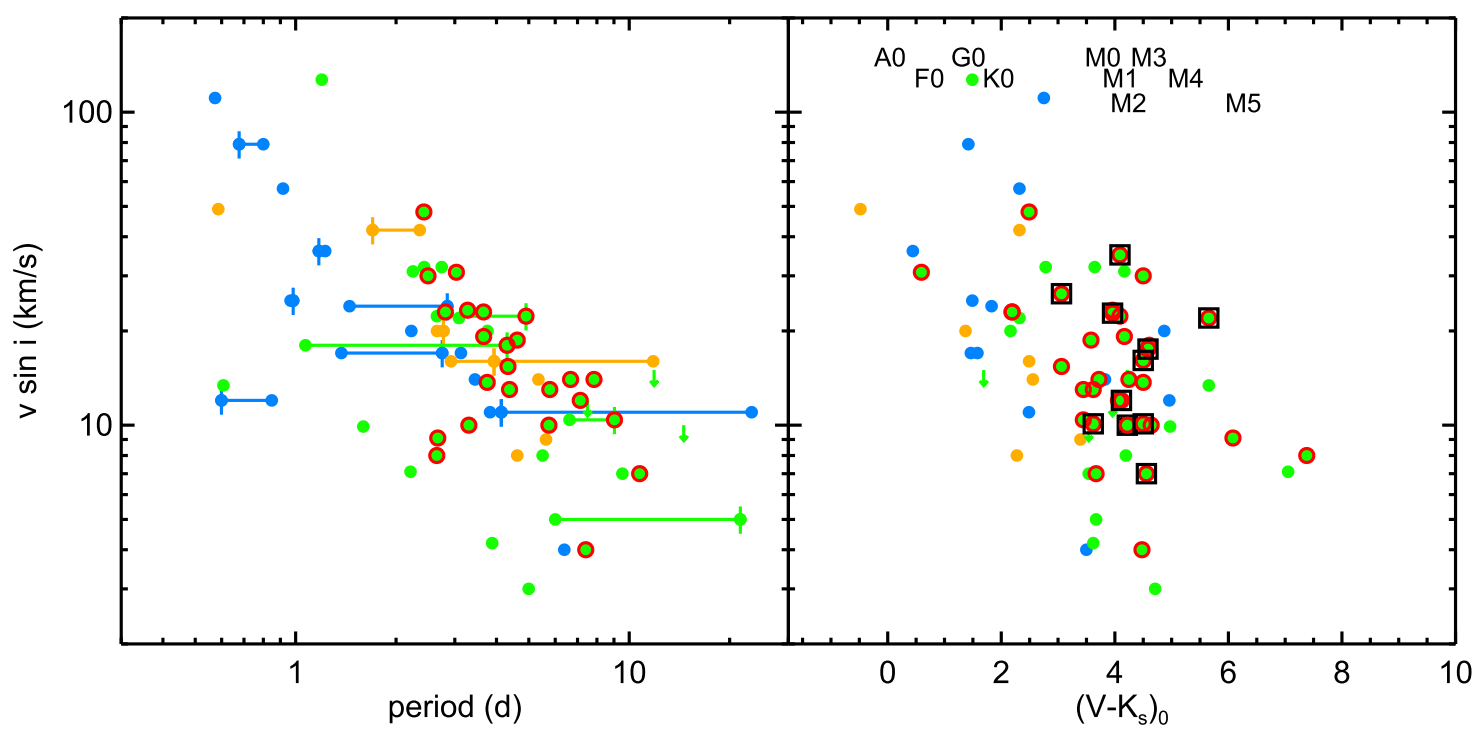

Figure 3. Comparison of periods obtained here to $v \sin i$ from the literature. Left panel: $v \sin i$ (from the literature) vs. period (as derived here). Right panel: $v \sin i$ (from the literature) vs. $\left(V-K_{\mathrm{s}}\right)_{0}$. The colors of the points are as follows: green $=$ highest-confidence member; orange $=$ possible member; and blue $=$ young, foreground population (see Section 2.2), where arrows denote upper limits. An additional red circle around a point denotes a high-confidence disk. In the left panel, the points linked by lines connect the first period (what we have taken as the rotation rate of the star, denoted by a small vertical line) with the fastest and slowest periods available for that star, on the assumption that the $v \sin i$ may not correspond to the first period for those stars with multiple periods. In the right panel, an additional square around a point indicates that we found no period for this star in the $\mathrm{K} 2$ data. There is no obvious trend for the stars lacking periods to have systematically higher or lower $v \sin i$ than those with periods.

and, for a few, significant disagreement with the periods derived from the high-quality $\mathrm{K} 2$ data.

The study with which we have the most disagreement is the Kilodegree Extremely Little Telescope (KELT) analysis (Rodriguez et al. 2017b), where 19/26 do not match. Our analysis of other clusters in common with KELT produced a much better match in periods. Perhaps this is not surprising, in particular for those stars still actively accreting or where the distribution of spots may be changing on relatively short timescales; changes in the LC shape of an accreting object can reasonably be expected to change on $\sim$ year timescales even though the stellar rotation rate itself does not change on short timescales.

In any event, for each mismatched period, we investigated the prior period and believe that the period(s) we report is/are the correct periods for these stars during the $\mathrm{K} 2$ campaign(s).

\subsection{Comparison to Literature $\mathrm{v} \sin \mathrm{i}$}

As discussed above in Section 2.2, it is likely that the relatively low fraction of Taurus members with periods in the $\mathrm{K} 2$ data is due to disk contributions to the LCs, making periods difficult to extract. To determine whether or not this results in a bias in our derived period $(P)$ distribution, we compared our periods with the projected rotational velocities $(v \sin i)$ from the literature. We started with the $v \sin i$ compilations in Rebull et al. (2004) and Güdel et al. (2007) and then added those from Nguyen et al. (2009).

Out of the Taurus members or the foreground population with K2 LCs, there are 51 with $v \sin i$ measurements or limits in the literature. Figure 3 compares the $v \sin i$ and the periods measured here. For stars with multiple measured periods, the measured $v \sin i$ in the literature may not correspond to the period we have taken as the rotation period. Thus, those stars with multiple periods are indicated in the plot. Disks and stars lacking periods in the $\mathrm{K} 2$ data are indicated.
In the left panel of Figure 3, we recover the expected relationship in that stars with fast $P$ have large $v \sin i$. Stars with disks lack shorter periods ( $<2$ days; see Section 4 below). In the right panel of Figure 3, there is no obvious trend for the stars lacking periods to have systematically higher or lower $v \sin i$ than those with periods. We conclude that our sample with periods is not significantly biased by omitting the $\sim 20 \%$ of (member) stars (nearly all of which have disks) that do not have periods, and comparisons to other, older clusters should be straightforward.

\section{5. $C M D s$}

Figures 4-6 are various versions of the $K_{\mathrm{s}} /\left(V-K_{\mathrm{s}}\right) \mathrm{CMD}$ for the entire sample with each highlighting the stars of interest: the most likely Taurus members, the possible Taurus members, and the older foreground population. Figure 4 shows the observed CMD, and Figures 5 and 6 show dereddened CMDs. Figures 4 and 5 include apparent and absolute $K_{\mathrm{s}}$, but not every star has a Gaia distance (see statistics in Table 1), so Figure 6 shows just apparent magnitudes. Figure 6 also distinguishes the periodic from the disked stars. By inspection of these CMDs, we primarily see that reddening is important, and the foreground population is older than the Taurus members.

\subsubsection{Taurus Members}

The observed CMD (Figure 4) shows a very large scatter for the Taurus members (both high-confidence and possible), most obviously in $\left(V-K_{\mathrm{s}}\right)$ color. Correcting for distance alone does not substantially reduce that scatter. (Correcting for distance makes large changes to the scatter of the NMs, and it becomes apparent that many NMs are giants.) When dereddened (Figure 5), the Taurus members (both high-confidence and possible) form a more recognizable cluster sequence, though still with scatter. Some of this scatter arises from uncertainty in the reddening correction, but some is likely intrinsic to the Taurus population. Although we are using $K_{\mathrm{s}}$, which can be 

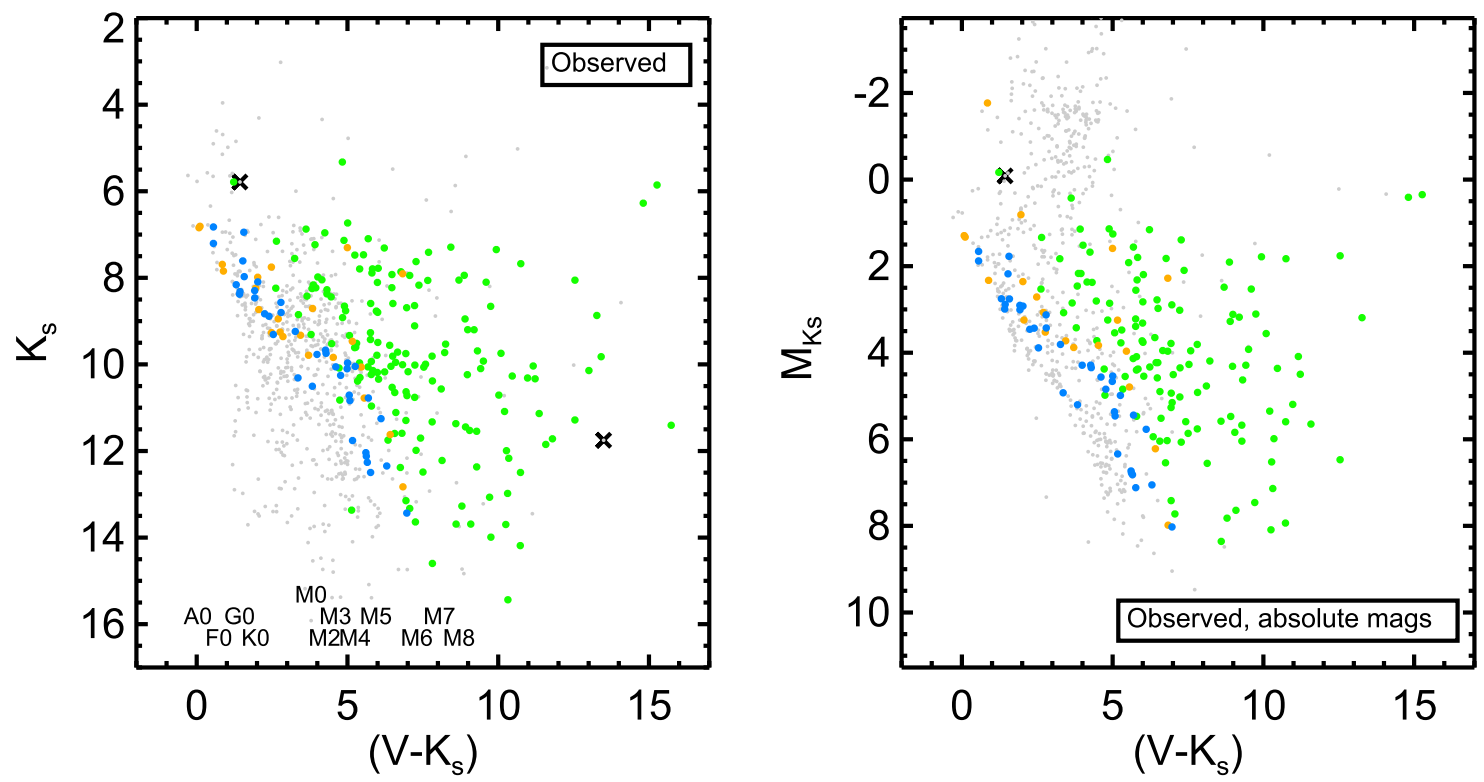

Figure 4. CMDs $\left(K_{\mathrm{s}}\right.$ vs. $\left.\left(V-K_{\mathrm{s}}\right)\right)$ for the sample for which we had or could infer $\left(V-K_{\mathrm{s}}\right)$ as described in the text (Section 2.3). In both plots, the colors are as follows: green shows highest-quality members; orange shows possible members; blue shows the foreground population; and gray shows those in the initial sample of candidate members (see Section 2.2). Points shown as $\times$ are too bright or too faint to yield periods from their LCs. Approximate spectral types for each $\left(V-K_{\mathrm{s}}\right)$ color are given in the first plot. Left panel: observed values. Note that Taurus members (highest-quality) are often highly reddened, and the foreground population has a considerably lower range of observed colors. Right panel: observed values but shifted for each object for which we had a Gaia DR2 distance (Section 2.3, sample fractions in Table 1) to convert the $y$-axis to absolute $K_{\mathrm{s}}$. In these observed CMDs, the members have a large dispersion toward the red. The foreground population is relatively well-behaved. The NM background includes a lot of giants, as revealed in the right-panel CMD using absolute magnitudes.
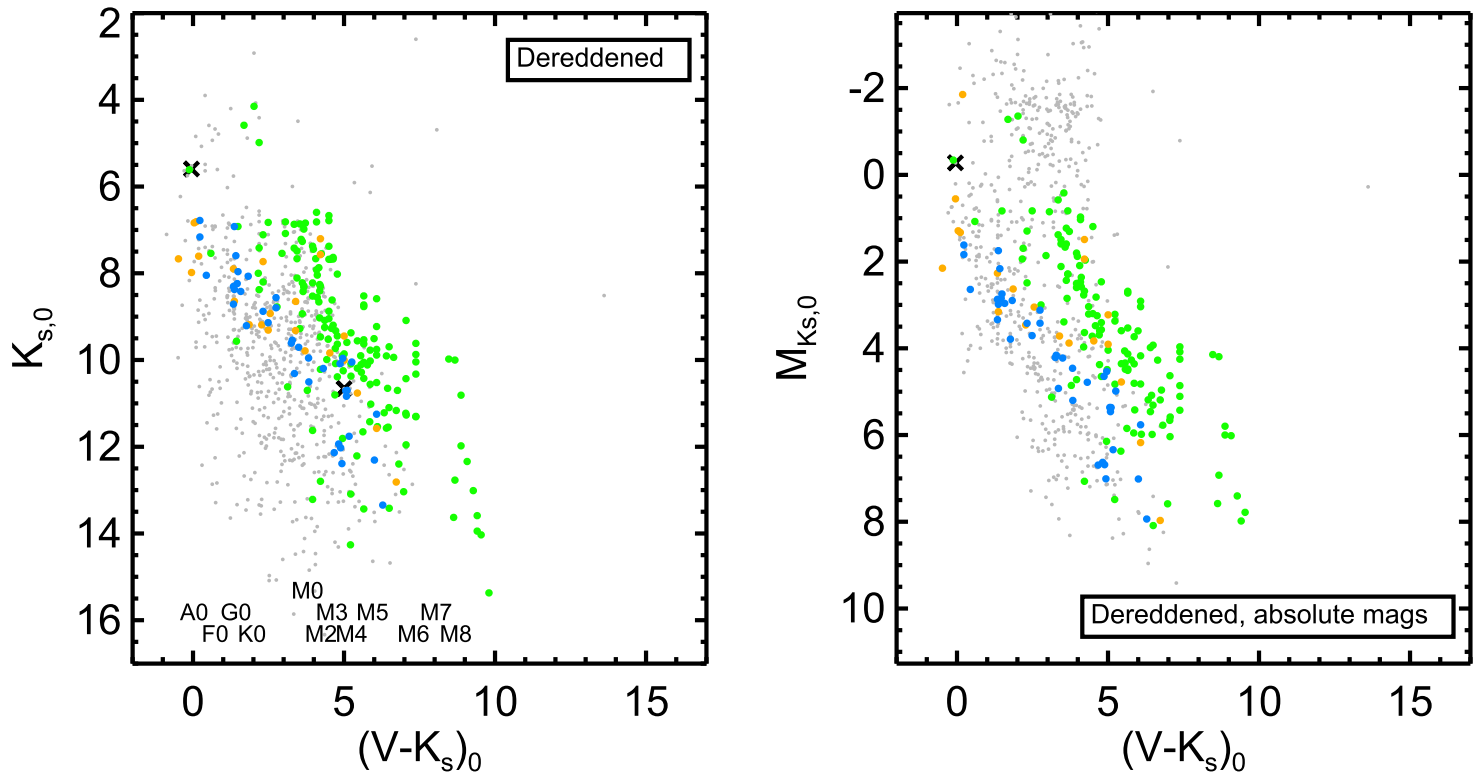

Figure 5. Dereddened CMDs $\left(K_{\mathrm{s}, 0}\right.$ vs. $\left.\left(V-K_{\mathrm{s}}\right)_{0}\right)$ for the sample. The notation is the same as in Figure 4, and note that the range on the $x$-axis is the same as that in Figure 4 as well. Left panel: dereddened (Section 2.5), observed values; right panel: dereddened absolute values. Correcting for reddening brings most of the stars bluer, and the change is large for many of the Taurus member stars in particular. The Taurus members are in a cluster sequence, but it is not as tight a relationship as in the older clusters (Papers I or IV) because the stars are so young. Some quantization can be seen as a result of the dereddening approach for some stars with spectral types (see Section 2.5). Little change occurs for the foreground population, as they are less subject to reddening than the Taurus members. They are lower in the CMD than the Taurus population, indicating that they are older.

affected by circumstellar disks, because this sample is defined by, and thus biased toward, targets with $\mathrm{K} 2 \mathrm{LCs}$, those stars with disks substantial enough to significantly affect $K_{\mathrm{S}}$ are generally also those that are so embedded as to not have a K2 LC. Table 2 (and its NM counterpart Table B1) includes an indication of where the IR excess starts, if an IR excess is detected. Less than $20 \%$ of the highest-quality member are likely to have their $K_{\mathrm{s}}$ value affected by disk excesses.

The relative lack of highest-confidence members toward the higher masses $\left(\left(V-K_{\mathrm{s}}\right)_{0} \lesssim 4\right)$ noted in Section 2.2 is apparent; many of the possible members (and for that matter, foreground population; see below) do fall in this color range. 

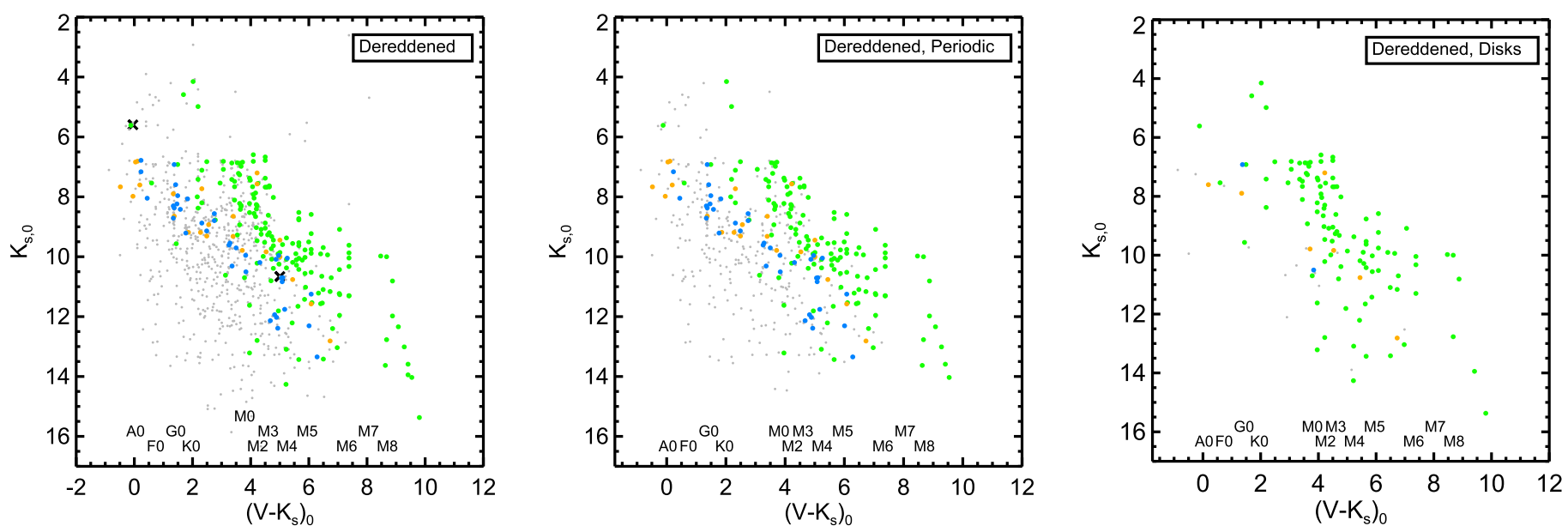

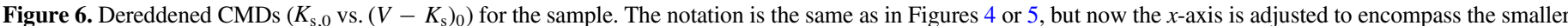

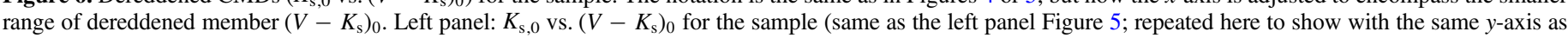

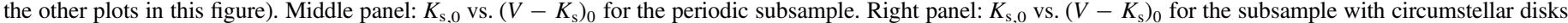

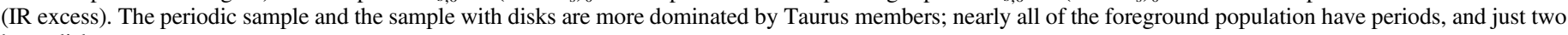
have disks.

Figure 6 highlights the periodic and disked samples in the dereddened CMD. (See statistics in Table 1.) Because a high fraction of the members (high-confidence and possible) are periodic, most of the Taurus stars appearing in the dereddened CMD also appear in the dereddened CMD of stars with periods. Because a lower fraction of the NMs have periods, many NMs do not appear in the CMD of stars with periods; in general, if a target has a period, it is more likely to be young. Similarly, a high fraction of the disked population are also members (most often members of Taurus, as opposed to the foreground population).

\subsubsection{Foreground Population}

In contrast to the Taurus members in Figure 4, there is not very much scatter in the foreground population's observed CMD; these stars, on average, have low extinction. The foreground sample is in front of most of the gas and dust associated with Taurus, so this tight cluster sequence is not surprising. In both Figures 4 and 5, the foreground population is also clearly lower in the CMD than most of the Taurus members (from either confidence level), indicating that this foreground population is older than that of Taurus.

In Figure 6, the periodic subsample retains all but one of the stars considered part of the foreground population. The fact that they have measurable periods means they likely have big spots and thus are likely young, but they are not Taurus members (see Section 2.2). Just two of the foreground population have a disk (one possible disk, one unambiguous disk); this low disk fraction is another indication that the foreground population is older than the Taurus members.

\section{Disks and Rotation}

Paper V presented LCs from USco that provide direct evidence for disk locking, with a striking clumping of disked stars near $\sim 2$ days, particularly for the later spectral types. Figure 7 shows period $(P)$ versus IR excess ([3.5]-[12]), for $\rho$ Oph ( 1 Myr; data from Paper V) and Taurus ( 3 Myr; just the highest-quality member sample). There are comparable numbers of stars in these two clusters and likely comparable uncertainties leading to $\left(V-K_{\mathrm{s}}\right)_{0}$, though we believe that we have done a slightly better job of dereddening in Taurus than in $\rho$ Oph (see Section 2.5). Uncertainties in $P$ should be low, and reddening should not significantly affect the [3.5]-[12] excess, but reddening will affect which bins (which panel in the plot) encompass which targets.

The relationship between $P$ and color seen in USco (Paper V) is not nearly so convincing in $\rho$ Oph. However, in Taurus, the relationship is clearer; the disked stars are clumped near $\sim 2$ days, and there are very few stars that both rotate more quickly than $\sim 2$ days and have disks. Taurus resembles USco more than $\rho$ Oph, which makes sense, as Taurus is older than $\rho$ Oph (and younger than USco). Moreover, $\rho$ Oph may be too young for disk locking to dominate (e.g., Hartmann 2002). As noted above, Figure 3 also suggests that the stars with disks rotate more slowly.

More detailed statistical tests reinforce what is seen by eye. For USco, all statistical tests find significant differences (Komolgorov-Smirnov [K-S] and Anderson-Darling for just the period distributions; 2D two-sided $\mathrm{K}-\mathrm{S}$ for the full distribution of $P$ versus $\left.\left(V-K_{\mathrm{s}}\right)_{0}\right)$. For the other two clusters, the disked and non-disked star samples are small enough that the results are just not as clear. In $\rho$ Oph, the 2D distributions that are most significantly different are for $2<\left(V-K_{\mathrm{s}}\right)_{0}<4$ (the upper right panel), but there are only four non-disked stars, so this is not a very robust result. In Taurus, the $P$ distribution alone for the non-disked sample is close to that for the disked stars; there is a clumping of the black points near $\sim 3-5$ days, which is most apparent in the $2<\left(V-K_{\mathrm{s}}\right)_{0}<4$ bin (the upper right panel of Figure 7). The 1D $P$ distributions are the most different for the $\left(V-K_{\mathrm{s}}\right)_{0}<2$ sample, but there are very few stars. The 2D distributions are significantly distinct between the disked and non-disked populations in each panel, but more ambiguously than for USco. (USco $2 \mathrm{D} \mathrm{K}-\mathrm{S}$ for the $5<\left(V-K_{\mathrm{s}}\right)_{0}<7.5$ bin has a probability of $\sim 2 \times 10^{-20}$ that they come from the same parent population; the same test for the same Taurus bin is $\sim 0.001$. The same test for the same color range but for $\rho$ Oph is just 0.23.)

We conclude that, within the constraints of the relatively small number of stars and the uncertainty in $\left(V-K_{\mathrm{s}}\right)_{0}$, there is some evidence that disks affect the rotation rate distribution in Taurus. The distribution of rotation rates versus color in Taurus 

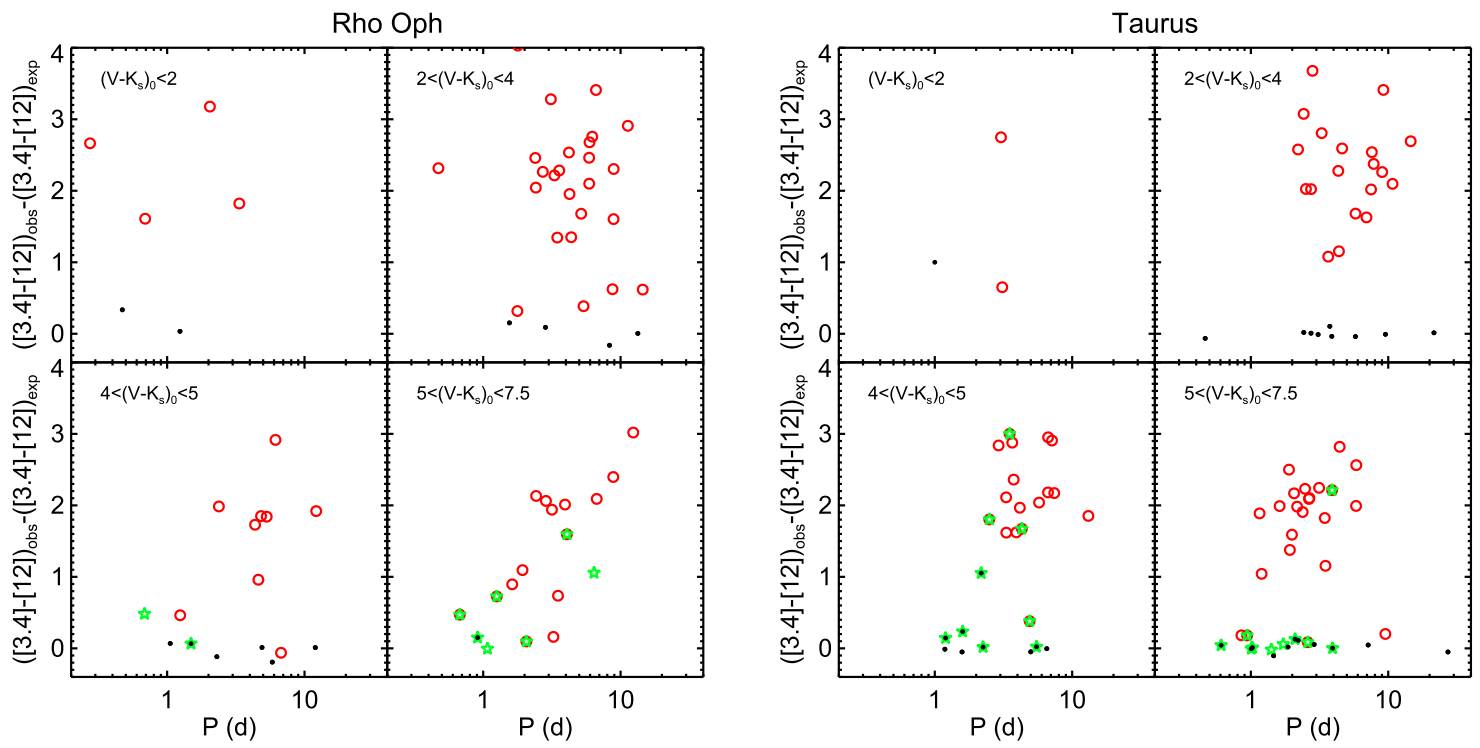

Figure 7. Observed [3.4]-[12] minus the expected photospheric [3.4]-[12] (see Section 2.4) vs. $P$ for stars in $\rho$ Oph (left panels) and Taurus (right panels; highestconfidence members only), differentiating between the high-confidence disked stars (red, open circles) and the high-confidence non-disked stars (small black dots). M stars are shown in the bottom panels; the left bottom panel, $4<\left(V-K_{\mathrm{s}}\right)_{0}<5$, is roughly M0-M3, and the right bottom panel, $5<\left(V-K_{\mathrm{s}}\right)_{0}<7.5$, is roughly M4M5. An additional green star in these panels denotes that it has more than one period (and is not tagged a pulsator), e.g., a likely binary; see the text. The relationship in $\rho$ Oph is far less clear than that in USco, because there is far more uncertainty in $\left(V-K_{\mathrm{s}}\right)_{0}$, and there are far fewer stars in comparison to USco. Although uncertainty in $\left(V-K_{\mathrm{s}}\right)_{0}$ and far fewer stars also affect Taurus, the relationship in Taurus more clearly resembles that in USco than in $\rho$ Oph; Taurus is older than $\rho$ Oph and younger than USco.

more closely resembles that from USco than that from $\rho$ Oph. We note also that there seem to be few binaries with disks in the lower-mass bin in Taurus, but with the relatively few stars available, it is hard to assess the significance of this in comparison to the other clusters.

We also note that all but one of the highest-confidence Taurus members that lack a period from K2 also have disks, supporting the idea that stochastic contributions from the disk can make periods harder to find.

\section{Period-Color Distributions}

In this section, we investigate $P$ as a function of $\left(V-K_{\mathrm{s}}\right)_{0}$ and put Taurus (and the foreground population) in context with the other clusters we have studied with K2 data. As in our other $\mathrm{K} 2$ rotation papers, for stars with more than one period, we have taken the first period and the measured $\left(V-K_{\mathrm{s}}\right)_{0}$ as representative of the same star (likely the primary if it is a multiple); both the assumed $\left(V-K_{\mathrm{s}}\right)_{0}$ and first period are listed in Table 2. Even if the star is a designated multiple identified from additional periods and position in the CMD, we do not include subsidiary companions separately in this analysis. Additionally, as before, we assume that the stars in these three clusters represent snapshots in time of the same population (see Coker et al. 2016).

\subsection{Distribution of $\mathrm{P}$ versus $\left(V-K_{s}\right)_{0}$}

Figure 8 shows $P$ versus $\left(V-K_{\mathrm{s}}\right)_{0}$. The left panel has the foreground older population; the others have both the highestconfidence members and the possible members. Overall, the slowest rotators are found in the early $\mathrm{M}$ stars and the fastest rotators are found in both the highest masses, A stars and F stars, and in the lowest masses we have here, mid-M stars. Despite the far fewer stars available from the foreground population, it can be seen, on average, that they are rotating more quickly than the (younger) Taurus members, consistent with their older ages. The disked stars are, on average, rotating more slowly than the disk-free Taurus members, consistent with disk locking (see Section 4 above). The latest $\mathrm{M}$ stars in Taurus are the youngest $\mathrm{M}$ stars yet studied with highquality LCs.

Figure 9 puts the $P$ versus $\left(V-K_{\mathrm{s}}\right)_{0}$ for Taurus and the foreground population in context with the other young clusters that have periods we derived from K2 LCs: $\rho$ Oph $(\sim 1 \mathrm{Myr}$; Paper V), USco ( $\sim 8 \mathrm{Myr}$; Paper V), Pleiades ( $125 \mathrm{Myr}$; Papers I-III), and Praesepe ( 790 Myr; Paper IV).

There are relatively few stars in Taurus (and even fewer foreground stars) in comparison with the older clusters. The shortest periods in all of these clusters are on the order of hours and are limited by breakup (see discussion in Paper V). The longest periods found in the youngest clusters are those of early $\mathrm{M}$ stars and are $\sim 10-20$ days; there are many periods longer than $\sim 10$ days in Praesepe, encompassing K and early M stars. At older ages, there are two well-defined sequences: rotation period increasing (slower rotation) to lower mass for FGK stars, and a second sequence where rotation period decreases sharply (faster rotation) as mass decreases among the $\mathrm{M}$ dwarfs. These two trends (particularly the latter) appear to already be in place by Taurus's age ( 3 Myr). For higher-mass stars $\left(\left(V-K_{\mathrm{s}}\right)_{0} \lesssim 3\right)$, the trend is for higher masses to have faster rotation rates (smaller $P$ ), and lower masses to have slower rotation rates, although there are very few stars available. For low-mass $\left(\left(V-K_{\mathrm{s}}\right)_{0} \gtrsim 3\right)$ stars, there are shorter periods at lower masses. Such trends are harder to see in the $\rho$ Oph sample, perhaps because of larger uncertainties in the colors (due to larger and more variable reddening). It does seem to be there in the Taurus foreground sample as well ( $\sim 30 \mathrm{Myr})$. There is an apparent decrease in the range of periods at a given color/mass as a function of age; this is at least partially (perhaps completely) due to the larger uncertainties in the inferred colors at younger ages. The $\left(V-K_{\mathrm{s}}\right)_{0}$ colors are more uncertain in the youngest clusters due to the larger 


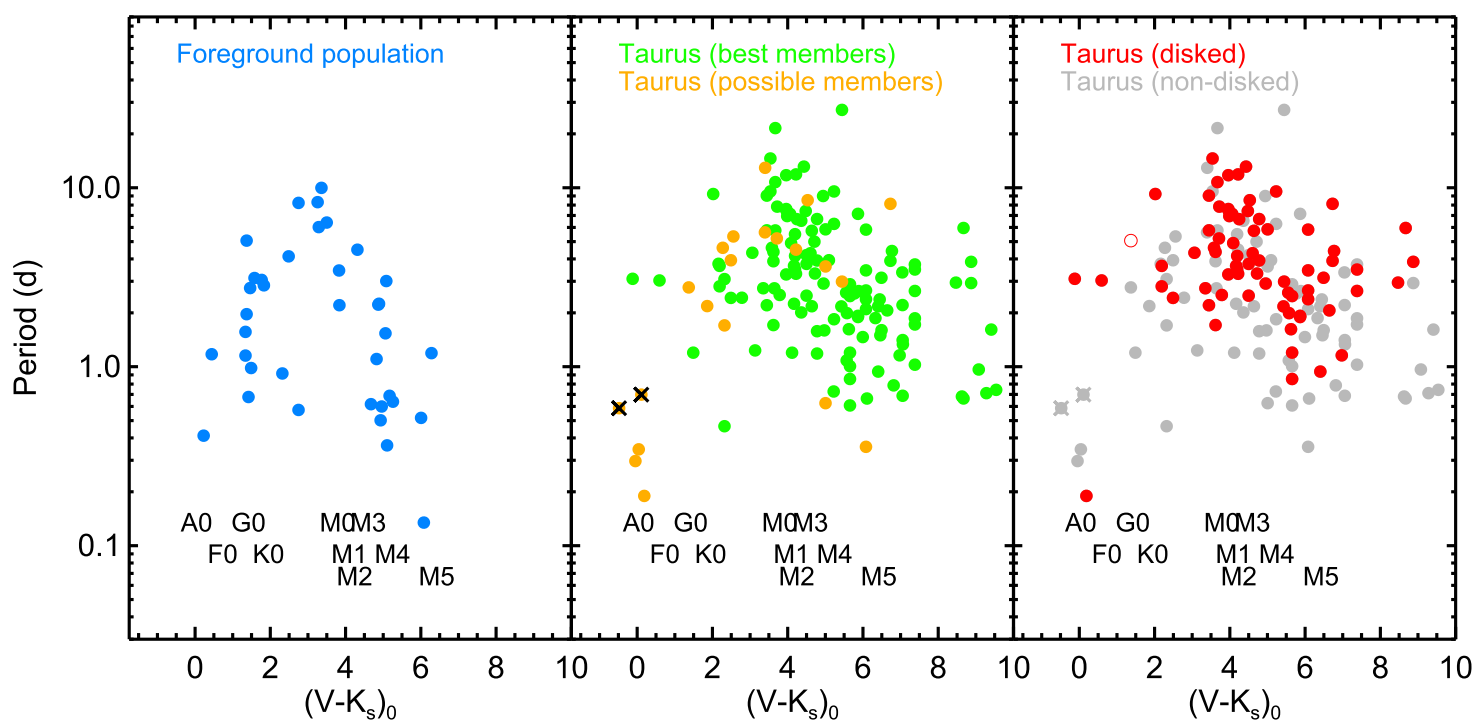

Figure 8. $P$ vs. $\left(V-K_{\mathrm{s}}\right)_{0}$. Left panel: (blue) foreground population. Middle panel: green $=$ highest-quality Taurus members; orange $=$ lower-confidence Taurus members; an additional $\times$ denotes stars that we believe to be pulsators. Right panel: red is disked, and gray is non-disked, for best and possible Taurus members (the single high-confidence disk among the foreground population is a hollow red circle). Approximate spectral types are indicated for reference. The slowest rotators are found in the early M stars; fast rotators are found in the A, F, and G stars and in the mid-M stars, the latest type we have here. Stars from the foreground population, on average, rotate more quickly than the younger Taurus members. Stars with disks, on average, rotate more slowly than stars without disks.

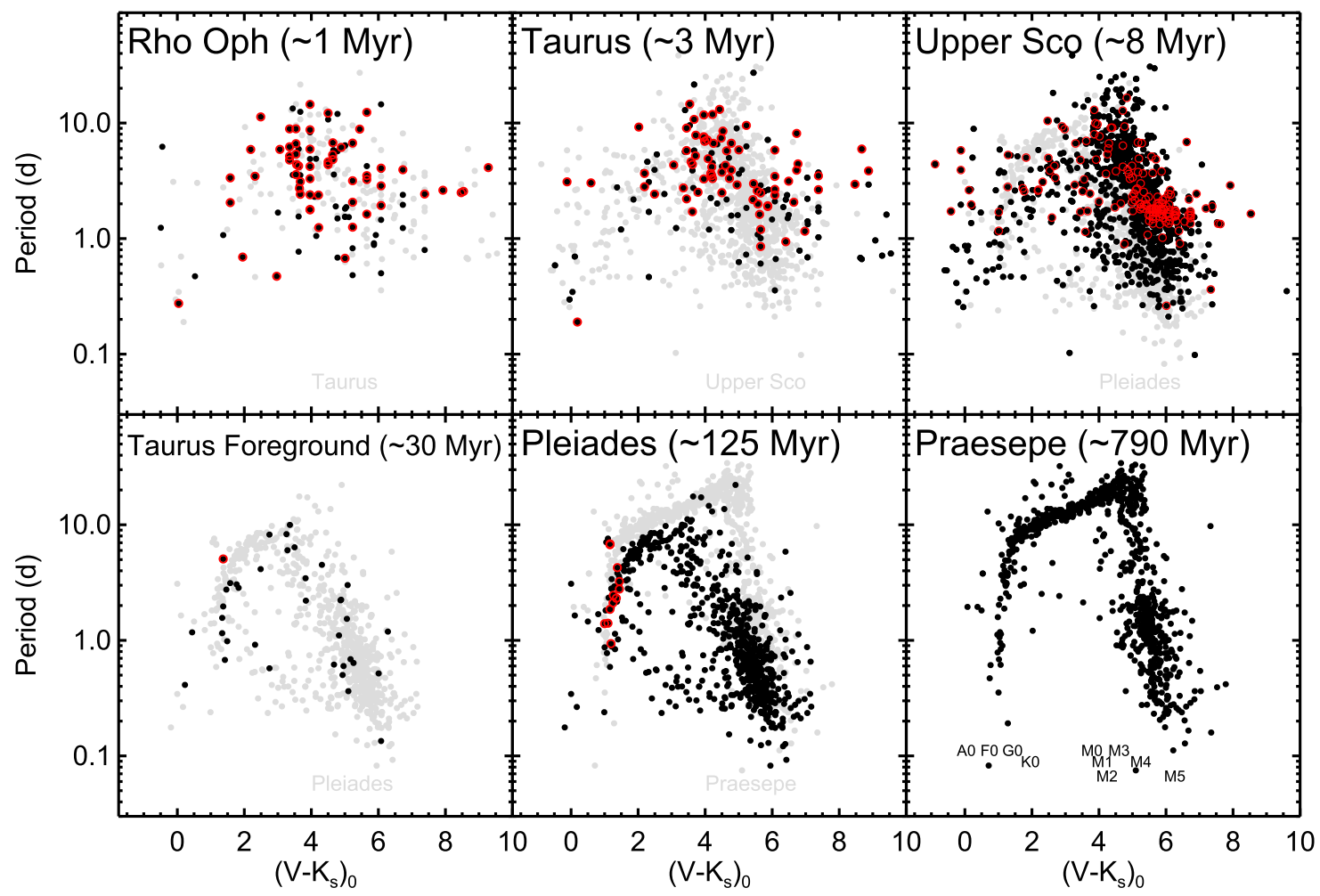

Figure 9. $P$ vs. $\left(V-K_{\mathrm{s}}\right)_{0}$ for stars in Taurus and the foreground population ("Taurus Foreground") in context with the other clusters with K2 periods. In each panel, the black dots are members, and the gray dots are members from the next panel-e.g., the Taurus sample has the USco sample in gray beneath it to aid in comparison between clusters. (The Pleiades appears for comparison in both USco and the Taurus foreground because the foreground population is just too sparse to provide meaningful comparisons to USco.) Additional red circles denote circumstellar disks; note that the Pleiades disks are all debris disks, while most of the disks in USco and all of those in Taurus and $\rho$ Oph are primordial disks. Upper left panel: $\rho$ Oph ( $\sim 1 \mathrm{Myr}$, from Paper V); upper center panel: Taurus ( $\sim 3 \mathrm{Myr})$; upper right panel: USco ( 8 Myr, from Paper V); lower left panel: Taurus foreground ( $30 \mathrm{Myr})$; lower center panel: Pleiades (125 Myr, from Papers I-III); lower right panel: Praesepe ( $\sim 790 \mathrm{Myr}$, Paper IV). Approximate spectral types are indicated for reference in the Praesepe panel. Errors on colors are conservatively estimated to be $\sim 1$ mag for $\rho$ Oph and Taurus, $\sim 0.4 \mathrm{mag}$ for USco and the Taurus foreground, and smaller than the points for the Pleiades and Praesepe. Recall that the apparent quantization of some stars' $\left(V-K_{\mathrm{s}}\right)_{0}$ (most apparent in $\rho$ Oph and some Taurus) is a result of our dereddening to the expected $\left(V-K_{\mathrm{s}}\right)$ color for that spectral type when that method of dereddening was the best option (see Section 2.5). The Taurus distribution has a lot of scatter but is starting to resemble the USco distribution. The (sparse) Taurus foreground distribution is consistent with the Pleiades distribution. 
extinction corrections, possibly the influence on $K_{\mathrm{s}}$ from the disk (this is relatively rare in this sample), and possibly due to variable extinction or accretion (and the non-simultaneity of the observed or inferred $V$ and measured $K_{\mathrm{s}}$ ). The Taurus distribution is not as organized as USco, but it is also not as disorganized as $\rho$ Oph. The Taurus distribution is starting to resemble the USco distribution, but it still has a lot of scatter, particularly in the $\mathrm{M}$ stars, more like $\rho$ Oph. The Taurus foreground distribution is sparse indeed but consistent with the Pleiades distribution.

The influence of disks can be seen in Figure 9; the top panels show all primordial disks, and the "pile-up" at $\sim 2$ days is obvious in USco but less so in the younger clusters (see Section 4 above). The disks in the Pleiades are all debris disks; the debris disk sample is likely incomplete among the $\mathrm{M}$ stars in Pleiades and Praesepe, since the more subtle IR excesses indicative of debris disks are harder to find among the fainter stars.

\section{Linkages to Analysis in Papers I-V}

Although the high-quality $\mathrm{K} 2$ data enable us to derive reliable periods for active young stars, the presence of disks makes a difference in the rotation rates and the LC shapes in clusters younger than $\sim 10 \mathrm{Myr}$. There are some similarities between the LCs we find in Taurus (and the foreground population) and LCs in the other clusters. In this section, we provide an assessment of the properties of the Taurus LCs in the same fashion as our other papers.

\subsection{Amplitudes}

We included in Papers I (Pleiades) and IV (Praesepe) information about the variability amplitude, where we define amplitude as the difference in magnitude between the 10th and the 90th percentile of the flux distribution for a given LC. We did not include such a discussion in Paper V (USco and $\rho$ Oph) because the systematics in that campaign (C2) were large and hard to manage (e.g., the fraction of the variability due to the instrument or due to the star is hard to determine). In most cases, however, the amplitudes we measured for the "best" LC for the periodic sources in USco and $\rho$ Oph are probably usable in the ensemble, especially given the relatively large uncertainty in $\left(V-K_{\mathrm{s}}\right)_{0}$. The instrumental systematics in the Taurus campaigns are much lower than that in $\mathrm{C} 2$, so here again, the amplitudes are probably due primarily to the astrophysical source.

Figure 10 shows the amplitudes of periodic variability (10th to 90 th percentiles) as a function of color and period for our K2 young clusters. As in Figure 9, the echoes of structures seen in the older and more populated cluster plots can be seen in the younger clusters. The average LC amplitude decreases with cluster age.

Stars with primordial disks have larger amplitudes, on average, though disked and non-disked stars both have a wide range of amplitudes in the youngest three clusters ( $\rho$ Oph, Taurus, and USco). The variability amplitudes for the Taurus disked stars primarily measure the larger amplitude variability due to accretion bursts ("bursters") or variable extinction ("dippers"). The variability amplitudes for the non-disked stars (and for the low-mass stars in the older clusters in Figure C1) primarily measure non-axisymmetrically distributed spots as they rotate into and out of our line of sight.
The earliest spectral type stars sampled here (A and F stars) have lower amplitudes, on average, than their same-age counterparts among the $\mathrm{G}, \mathrm{K}$, and $\mathrm{M}$ stars. This makes sense since the $\mathrm{A}$ and $\mathrm{F}$ stars are expected to have substantially less fractional spot coverage than the $\mathrm{G}, \mathrm{K}$, and $\mathrm{M}$ stars at any given age.

We expected that longer period rotators might have lower amplitudes on average, due to lower levels of activity. That is very roughly the case at the ages of Praesepe and perhaps Pleiades; it might be the case at the age of USco, and it seems to not be the case for disk-free younger stars.

There is obvious substructure seen in the amplitude distribution against color or period in Praesepe (and more subtle echoes of it in the Pleiades) as a function of $\left(V-K_{\mathrm{s}}\right)_{0}$, some of which may be selection effects in that the longest periods may require larger amplitudes for detection (Paper IV). The substructure seen in the amplitude distribution in the Pleiades and Praesepe is not obvious in the clusters $<100 \mathrm{Myr}$ - perhaps the behavior is not yet organized enough, or there are not enough stars for comparison. That lack of structure could be explained in the $\left(V-K_{\mathrm{S}}\right)_{0}$ plot by invoking the likely large uncertainties in $\left(V-K_{\mathrm{s}}\right)_{0}$ for the young clusters, but the uncertainty in $P$ is likely small, so the relative lack of substructure in the distributions as a function of $P$ for the younger stars is likely real, though greatly complicated by the range of masses included at any given $P$ (or amplitude).

\subsection{LC and Periodogram Categories}

In Papers II, IV, and V, we classified the LC and periodogram morphologies for the Pleiades, Praesepe, USco, and $\rho$ Oph; see these references for discussion of the classes (e.g., double-dip, scallop, etc.). Table 3 includes statistics on these classifications for all of the clusters, now including Taurus.

A high fraction of the Taurus stars are periodic, $83 \%$. As for nearly all of the other clusters, $\sim 70 \%$ of the periodic Taurus members have only one period; about $30 \%$ of the periodic sample has at least two real periods. The Taurus foreground has different proportions, but it is likely biased/incomplete.

There are some aspects of the periodic LC morphologies that are common between the clusters and others that show signs of evolution with stellar age. Specifically:

1. The fraction of double-dip stars falls substantially between Praesepe and $\rho \mathrm{Oph}$. Assuming that the rate at which we find double-dip characteristics increases as rotation period decreases (e.g., Basri \& Nguyen 2018), this makes sense since Praesepe is, on average, rotating much more slowly than $\rho \mathrm{Oph}$.

2. LCs of the scallop shell morphology (and related categories) continue to be rare but much more common among the younger clusters. None have disks. We note that Zhan et al. (2019) find scallop shell stars at $~ 40 \mathrm{Myr}$, but there are very few by $\sim 125 \mathrm{Myr}$, the age of the Pleiades.

3. LC categories suggestive of complicated spot occurrence and evolution (beater, complex peak) seem harder to find in clusters with a higher disk fraction. In contrast, shape changers (which could arise from spot/spot group 

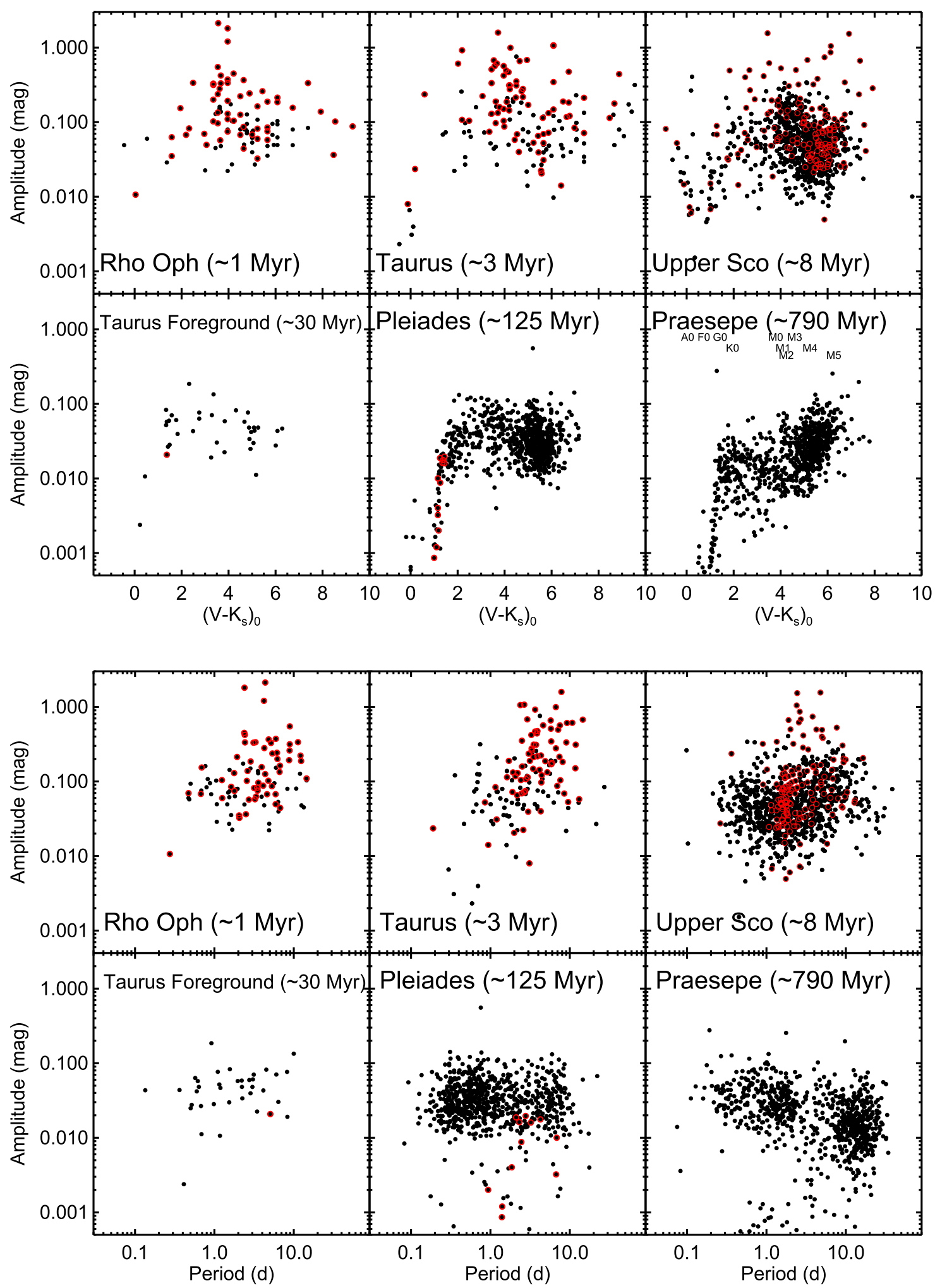

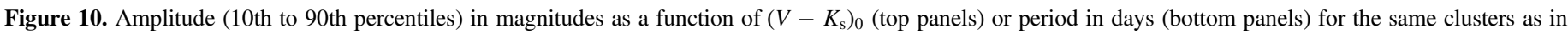

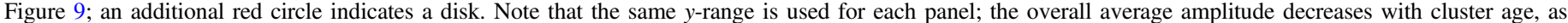

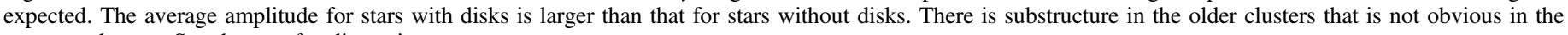
younger clusters. See the text for discussion.

emergence/evolution or from disk influence) are more common in clusters with a higher disk fraction.

4. Resolved distant peaks (which are most likely binaries, either real or apparent) occur at comparable rates across all clusters. Pulsators are rare in all clusters, which is unsurprising, at least in the stellar mass range where our studies are focused.

5. Dippers and bursters only occur in clusters that still have primordial disks, consistent with our physical interpretation of these phenomena as interactions with gas/dust disks. The 
Table 3

Star/LC/Periodogram Categories

\begin{tabular}{|c|c|c|c|c|c|c|c|c|c|c|c|c|c|c|c|c|c|c|c|c|c|c|}
\hline \multirow{2}{*}{ Category } & \multicolumn{3}{|c|}{ Praesepe } & \multicolumn{4}{|c|}{ Pleiades } & \multicolumn{3}{|c|}{ Tau F'gnd } & \multicolumn{4}{|c|}{ USco } & \multicolumn{4}{|c|}{ Taurus } & \multicolumn{4}{|c|}{$\rho$ Oph } \\
\hline & $(1)^{\mathrm{b}}$ & $(2)^{\mathrm{c}}$ & $(3)^{\mathrm{d}}$ & $(1)^{\mathrm{b}}$ & $(2)^{\mathrm{c}}$ & $(3)^{\mathrm{d}}$ & $(4)^{\mathrm{e}}$ & $(1)^{\mathrm{b}}$ & $(2)^{\mathrm{c}}$ & $(3)^{\mathrm{d}}$ & $(1)^{\mathrm{b}}$ & $(2)^{\mathrm{c}}$ & $(3)^{d}$ & $(4)^{\mathrm{e}}$ & $(1)^{b}$ & $(2)^{c}$ & $(3)^{d}$ & $(4)^{\mathrm{e}}$ & $(1)^{\mathrm{b}}$ & $(2)^{\mathrm{c}}$ & $(3)^{d}$ & $(4)^{\mathrm{e}}$ \\
\hline Member LCs & 938 & 1.00 & $\cdots$ & 826 & 1.00 & & $\cdots$ & 38 & 1.00 & $\cdots$ & 1136 & 1.00 & & .. & 179 & 1.00 & $\cdots$ & & 174 & 1.00 & $\cdots$ & \\
\hline Periodic & 819 & 0.87 & 1.00 & 759 & 0.92 & 1.00 & 1.00 & 37 & 0.97 & 1.00 & 974 & 0.86 & 1.00 & 0.81 & 148 & 0.83 & 1.00 & 0.71 & 106 & 0.61 & 1.00 & 0.73 \\
\hline Single period & 674 & 0.72 & 0.82 & 598 & 0.72 & 0.79 & 0.50 & 25 & 0.66 & 0.68 & 756 & 0.67 & 0.78 & 0.68 & 108 & 0.60 & 0.73 & 0.58 & 85 & 0.49 & 0.80 & 0.61 \\
\hline Multi-period & 145 & 0.15 & 0.18 & 161 & 0.19 & 0.21 & 0.50 & 12 & 0.32 & 0.32 & 218 & 0.19 & 0.22 & 0.12 & 40 & 0.22 & 0.27 & 0.13 & 21 & 0.12 & 0.20 & 0.11 \\
\hline Double-dip & 163 & 0.17 & 0.20 & 107 & 0.13 & 0.14 & 0.25 & 6 & 0.16 & 0.16 & 133 & 0.12 & 0.14 & 0.09 & 12 & 0.07 & 0.08 & 0.05 & 6 & 0.03 & 0.06 & 0.04 \\
\hline Moving double-dip & 121 & 0.13 & 0.15 & 31 & 0.04 & 0.04 & 0.17 & 5 & 0.13 & 0.14 & 32 & 0.03 & 0.03 & 0.02 & 5 & 0.03 & 0.03 & 0.01 & $\cdots$ & $\cdots$ & $\cdots$ & $\cdots$ \\
\hline Shape changer & 297 & 0.32 & 0.36 & 114 & 0.14 & 0.15 & 0.25 & 16 & 0.42 & 0.43 & 277 & 0.24 & 0.28 & 0.43 & 83 & 0.46 & 0.55 & 0.58 & 47 & 0.27 & 0.44 & 0.45 \\
\hline Scallop/clouds? ${ }^{\mathrm{f}}$ & $\ldots$ & $\ldots$ & $\ldots$ & 5 & 0.01 & 0.01 & $\ldots$ & 1 & 0.03 & 0.03 & 28 & 0.02 & 0.03 & $\ldots$ & 6 & 0.03 & 0.04 & 0.00 & $\ldots$ & $\ldots$ & $\ldots$ & $\ldots$ \\
\hline Beater & 77 & 0.08 & 0.09 & 136 & 0.16 & 0.18 & 0.75 & 7 & 0.18 & 0.19 & 107 & 0.09 & 0.11 & 0.03 & 18 & 0.10 & 0.12 & 0.05 & 10 & 0.06 & 0.09 & 0.06 \\
\hline Complex peak & 68 & 0.07 & 0.08 & 89 & 0.11 & 0.12 & 0.42 & 2 & 0.05 & 0.05 & 8 & 0.01 & 0.01 & $\ldots$ & 6 & 0.03 & 0.04 & 0.02 & $\ldots$ & $\ldots$ & $\cdots$ & $\ldots$ \\
\hline Resolved, close peaks & 71 & 0.08 & 0.09 & 127 & 0.15 & 0.17 & 0.42 & 9 & 0.24 & 0.24 & 151 & 0.13 & 0.16 & 0.08 & 25 & 0.14 & 0.17 & 0.07 & 12 & 0.07 & 0.11 & 0.04 \\
\hline Resolved, distant peaks & 77 & 0.08 & 0.09 & 39 & 0.05 & 0.05 & 0.17 & 5 & 0.13 & 0.14 & 85 & 0.07 & 0.09 & 0.05 & 18 & 0.10 & 0.12 & 0.06 & 9 & 0.05 & 0.08 & 0.08 \\
\hline Pulsator & 17 & 0.02 & 0.02 & 8 & 0.01 & 0.01 & 0.00 & $\cdots$ & $\cdots$ & $\cdots$ & 13 & 0.01 & 0.01 & 0.01 & 2 & 0.01 & 0.01 & $\cdots$ & $\cdots$ & $\cdots$ & $\cdots$ & $\cdots$ \\
\hline Disk & $\ldots$ & $\cdots$ & $\ldots$ & 12 & 0.01 & 0.02 & 1.00 & $\cdots$ & $\ldots$ & $\ldots$ & 208 & 0.18 & 0.17 & 1.00 & 101 & 0.56 & 0.49 & 1.00 & 80 & 0.46 & 0.55 & 1.00 \\
\hline Dipper $^{\mathrm{g}}$ & $\ldots$ & $\cdots$ & $\ldots$ & $\ldots$ & $\ldots$ & $\ldots$ & $\ldots$ & $\ldots$ & $\ldots$ & $\ldots$ & 66 & 0.06 & 0.06 & 0.31 & 21 & 0.12 & 0.12 & 0.21 & 17 & 0.10 & 0.15 & 0.20 \\
\hline Burster $^{g}$ & $\cdots$ & $\cdots$ & $\cdots$ & $\cdots$ & $\cdots$ & $\cdots$ & $\cdots$ & $\cdots$ & $\cdots$ & $\cdots$ & 24 & 0.02 & 0.01 & 0.11 & 25 & 0.14 & 0.11 & 0.25 & 13 & 0.07 & 0.08 & 0.16 \\
\hline
\end{tabular}

Notes.

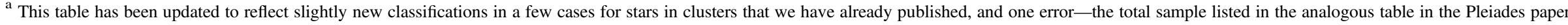
erroneously included just the best members as the total sample, but the rest of that table incorporated both the best plus "ok" members. The numbers in the present paper are correct.

${ }^{b}$ Total number of stars in the (sub)sample; for Taurus, it is the combination of the highest-quality and possible members. Examples for Praesepe: there are 938 members and 819 periodic members.

${ }^{\mathrm{c}}$ Sample fraction (fraction of the given type), out of the members. Examples for Praesepe: 87\% of the members are periodic; $72 \%$ of the members are single-period.

${ }^{\mathrm{d}}$ Periodic sample fraction (fraction of the given type), out of the periodic members. Examples for Praesepe: $82 \%$ of the periodic members are single-period; $18 \%$ of the periodic members are multi-periodic.

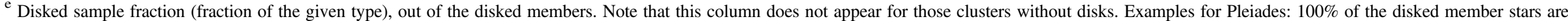
periodic; $50 \%$ of the disked member stars are single-period; $25 \%$ of the disked member stars are double-dip.

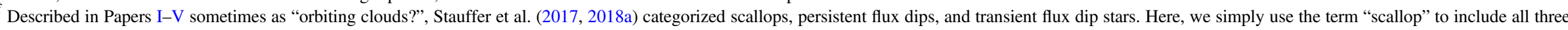
LC categories (Stauffer et al. 2017, 2018a).

${ }^{\mathrm{g}}$ Some objects are tagged burster or dipper but not periodic. The fraction of the periodic sample that is burster or dipper is correct, for the periodic subsample. 


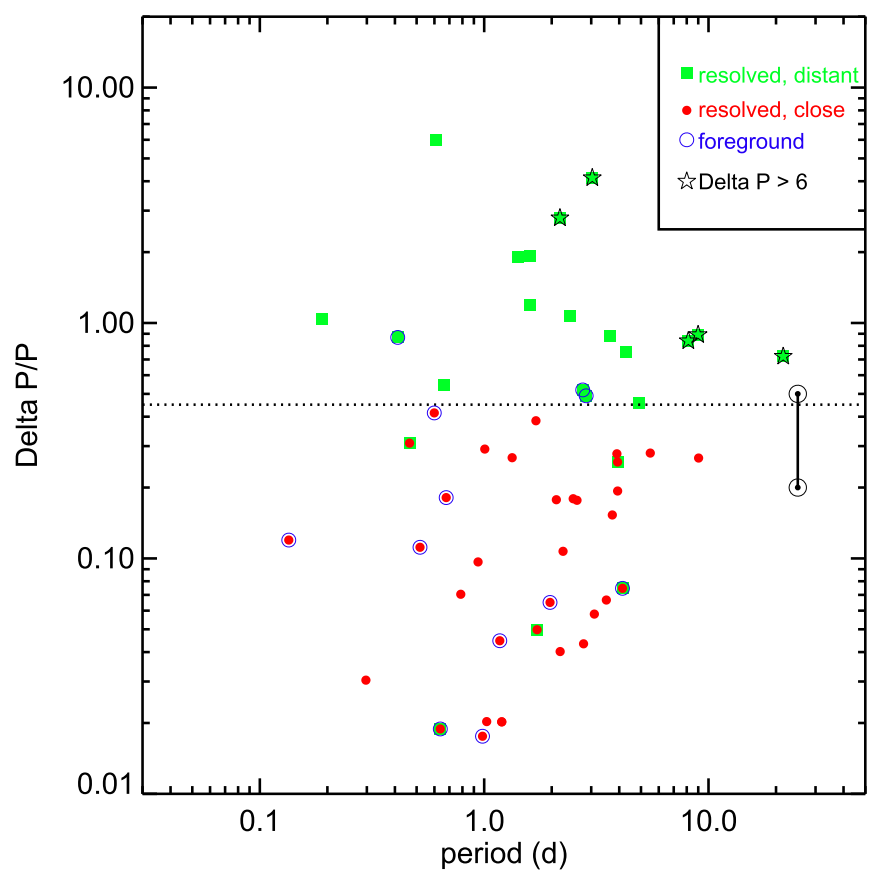

Figure 11. Plot of $\Delta P / P_{1}$ vs. $P$ for stars that are multi-periodic and with resolved distant peaks (green squares) and resolved close peaks (red dots) for high-confidence and possible members. An additional black star indicates that $|\Delta P|>6$ days. An additional blue circle means the star is from the older foreground population. The range of possible values for the Sun is included for reference $(\odot)$; if one takes as $\Delta P$ the range of periods measured where sunspots occur, $\Delta P / P_{1} \sim 0.1-0.2$, but if one takes the full range of $\Delta P$, equator to pole, $\Delta P / P_{1} \sim 0.5$. The dotted line is at $\Delta P / P_{1}=0.45$ and denotes the boundary between close and distant resolved peaks. There are far fewer structures in this diagram as compared to that for the Pleiades; it is possible that there are not enough complete cycles in the $\sim 70$ days campaign for extraction of clear multiple periods, or the disk influence on the LCs complicates derivation of the periods.

vast majority of dippers and bursters are also disked stars; a few have little or no IR excess, though they could still have gas disks depleted in dust.

\section{3. $\Delta \mathrm{P}$ Distributions}

As for the other clusters, we calculated the $\Delta P / P_{\text {rot }}$ metric for stars with resolved multi-period peaks; see Figure 11. This metric $\Delta P / P_{\text {rot }}$ is defined by taking the closest peak to the $P_{\text {rot }}$, subtracting the smaller from the larger, and dividing by the $P_{\text {rot }}$. For well-separated peaks and/or $M$ stars (see Paper V and Stauffer et al. 2018a), it is very likely that the two periods are two independent periods of two components of a binary system.

The distribution in Taurus, like that for USco/ $\rho$ Oph (Paper V) and Praesepe (Paper IV), has relatively little structure as compared to that for the Pleiades (Paper II). Here, as noted in Paper V, the $\sim 70$ days $\mathrm{K} 2$ campaigns are not quite long enough to capture multiple complete cycles in order to be able to resolve two close periods. If the phasing of the two periods is favorable, we can resolve the two periods, but if the phasing is not "lucky", $\sim 70$ days is not long enough to distinguish the periods.

\section{Summary and Conclusions}

We have presented the K2 LCs and periods derived from them for the Taurus star-forming region. We have LCs for about $30 \%$ of the highest-confidence Taurus members in the literature; most of the Taurus members are north of the region observed by $\mathrm{K} 2$. However, because the $\mathrm{K} 2$ region extends over a larger area, it enables the study not only of bona fide Taurus molecular cloud members, but also of a somewhat older, more dispersed population of candidate young stars.

We followed the methodology used in our earlier Papers I-V and find, again, that cluster membership is one of the most difficult parts of our analysis. We distributed all plausible candidate members of Taurus that were observed by K2 among four bins-highest-quality members, possible members, a population of stars in front of the Taurus members ("foreground") that are likely about three times older than Taurus members, and NMs. We retain, for further analysis, a sample consisting of the highest-quality members and the possible members. Dereddening is also challenging and likely contributes uncertainty to the $\left(V-K_{\mathrm{s}}\right)_{0}$ colors. We used IR data to identify stars with disks. We classified the LCs according to the classes we identified in Papers I-V. We retained up to four periods for each star.

We find slightly more than $80 \%$ of the Taurus members to be periodic; the foreground population is highly biased/incomplete, but nearly all of the foreground population is also periodic.

We find that the periods from our analysis are generally well matched to those from the (substantial) previous literature, except for possibly the KELT analysis (Rodriguez et al. 2017a). Our periods are also consistent with the available literature $v \sin i$ values, and our analysis suggests that there is no substantial bias in our sample introduced by our inability to get periods from stars with a high level of stochastic contributions (from, e.g., a disk or accretion).

Despite the relatively paucity of stars with K2 LCs and derived periods in Taurus (as compared to USco), similar relationships between rotation rate and disk presence are found in Taurus. Especially for the M stars, the periods are clumped near $\sim 2$ days, and there are very few stars that both have disks and rotate faster than $\sim 2$ days.

The latest $M$ stars in Taurus are the youngest $M$ stars thus far studied with high-quality LCs, extending into the brown dwarf regime.

The overall distribution of rotation rates as a function of color (as a proxy for mass) in Taurus, and in the foreground population, is similar to that found in other clusters: the slowest rotators are in the early $\mathrm{M}$ stars, and both the higher masses and lower masses are the fastest rotators. The member distribution looks similar to that from $\rho$ Oph and USco (Paper V), suggesting that the distribution (particularly for $\mathrm{M}$ stars) is already in place by the age of Taurus ( 3 Myr). The slightly older ( $\sim 30 \mathrm{Myr}$ ) foreground population has very few stars but is consistent with the USco and Pleiades distributions.

The LC amplitude distribution as a function of $\left(V-K_{\mathrm{s}}\right)_{0}$ or period has trends and substructure, not all of which are easy to interpret. The overall amplitude goes down as clusters age. Higher-mass stars have lower amplitudes than lower-mass stars; however, there is a selection effect in that the amplitudes of the faintest stars have to be larger to be detected. Stars with primordial disks have, on average, larger amplitudes, though the mechanisms driving the variability are different than for stars without disks. Interpretation of additional structure in these distributions is much less clear.

As for many of the other young clusters observed by $\mathrm{K} 2$, about $70 \%$ of the periodic members have just one period 
detected, and about $30 \%$ of the periodic members have more than one period. Scallop shell/orbiting dust cloud stars are still rare; there are just seven in the sample discussed here (one from the foreground and six from Taurus). LC categories that we have interpreted as spot/spot group changes (emergence/ evolution), namely beaters and complex peaks, seem harder to find in clusters with many primordial disks. Shape changers, which could be from spot/spot group changes or from disk interactions and/or accretion, are more common in clusters with a higher disk fraction. Dippers and bursters are only found in clusters with primordial disks; most of these specific objects have obvious dusty disks.

A. M. Cody et al. (2020, in preparation) will explore the range of LC properties of the disked members of Taurus. N. Roggero et al. (2020, in preparation) will explore the dipper population in Taurus.

Some of the data presented in this paper were obtained from the Mikulski Archive for Space Telescopes (MAST). Support for MAST for non-HST data is provided by the NASA Office of Space Science via grant NNX09AF08G and by other grants and contracts. This paper includes data collected by the Kepler mission. Funding for the Kepler mission is provided by the NASA Science Mission directorate. This research has made use of the NASA/IPAC Infrared Science Archive (IRSA), which is operated by the Jet Propulsion Laboratory, California Institute of Technology, under contract with the National Aeronautics and Space Administration. This research has made use of NASA's Astrophysics Data System (ADS) Abstract Service, and of the SIMBAD database, operated at CDS, Strasbourg, France. This research has made use of data products from the Two-Micron All-Sky Survey (2MASS), which is a joint project of the University of Massachusetts and the Infrared Processing and Analysis Center, funded by the National Aeronautics and Space Administration and the National Science Foundation. The 2MASS data are served by the NASA/IPAC Infrared Science Archive, which is operated by the Jet Propulsion Laboratory, California Institute of Technology, under contract with the National Aeronautics and Space Administration. This publication makes use of data products from the Widefield Infrared Survey Explorer, which is a joint project of the University of California, Los Angeles, and the Jet Propulsion Laboratory/California Institute of Technology, funded by the National Aeronautics and Space Administration.

Facilities: 2MASS, WISE, IRSA, Exoplanet Archive.

\section{Appendix A Membership}

\section{A.1. Members}

Despite the fact that Taurus is close $(\sim 140 \mathrm{pc})$ and has been studied for decades, membership is still controversial. Recent literature combines years of prior data collection with the Gaia DR2 data in an attempt to create a complete list of members (e.g., Luhman et al. 2017; Luhman 2018; Esplin \& Luhman 2019; Galli et al. 2019; and references therein). Numerous approaches have sought a dispersed population of young stars (e.g., Wichmann et al. 1996; Slesnick et al. 2006; Rebull et al. 2010; Rebull et al. 2011; Kraus et al. 2017; and references therein). The net result is persistently confusing in the literature, with a subset of stars that most authors agree must be members, stars where membership evidence is mixed, and still other stars where evidence is both mixed and controversial, particularly those having several indications of youth but with distinct motions and/or distances that are apparently inconsistent with membership.

In the context of this paper, we needed to find the sample of member stars that also had K2 LCs. It is important to note three important selection effects. First, K2 LCs were only downloaded for targets submitted in advance, so completeness is limited by the submitted list of targets. Second, the K2 observations did not cover all of the sky encompassing the least controversial Taurus members (see Figure 1, in addition to discussion below). Third, since it observed in optical bands, K2 effectively selected against those Taurus members that are so embedded as to attenuate the optical data (see discussion below and Section 2.4).

Based on our experience with the other K2 clusters that we previously analyzed (Papers I-V), we knew that we had to start with an overly generous list of possible members; by starting with an expansive set, we could analyze all of the LCs in the same way, at the same time (thus reducing biases in the analysis). Subsequent membership modifications implemented late in the process then become trivial to implement.

We assembled a list of 851 EPIC-numbered targets with LCs (e.g., targets submitted in advance that had actually been observed) that could be members of Taurus with data in either of the Taurus-relevant $\mathrm{K} 2$ campaigns ( $\mathrm{C} 4$ and $\mathrm{C} 13)$. These targets originated from any of the 64 different $\mathrm{K} 2 \mathrm{GO}$ programs that mention in their abstract studying young stars or members of Taurus. These programs did not necessarily perform complete and thorough vetting of possible targets prior to target submission (we note that both $\mathrm{C} 4$ and $\mathrm{C} 13$ predate Gaia DR2); observers just included targets that could be Taurus members, so we likewise included them in our initial target list assembly.

We proceeded with the analysis of those $\sim 850$ LCs in parallel with membership assessment efforts. For most of these targets (see Section 2.3 and Table 1), there are Gaia DR2 and 2MASS data. We explored a variety of parameter spaces such as vector point diagrams and CMDs. While this process was underway, Luhman (2018) was published, with similar subgroups as we had identified, but with a much more complete analysis than we had accomplished to that point. Subsequently, Esplin \& Luhman (2019) and Galli et al. (2019) were published, again presenting lists of members based primarily on Gaia DR2 data. These recent analyses include all of the least controversial Taurus members. We made the decision to adopt the intersection of those targets with K2 LCs and those in Esplin \& Luhman (2019) as the highest-quality members.

Note that the regions of sky encompassed by Luhman (2018), Esplin \& Luhman (2019), and Galli et al. (2019) are significantly different than the region of sky observed by $\mathrm{K} 2$ in the two campaigns including Taurus members (Figure 1). The K2 observations cover about $30 \%$ of the Taurus members from Esplin \& Luhman (2019), and the K2 observations also encompass a much larger region of the sky to the south of where the membership work was focused. Moreover, the Esplin \& Luhman (2019) member list seems to have very few highermass $\mathrm{G}$ and $\mathrm{K}$ stars. ${ }^{13}$ Since that mass range proved interesting for the rotation rate distribution in Papers I-V, we wanted to be

\footnotetext{
13 A similar G star deficit was noted also by Tokovinin \& Briceño (2020) in the context of the USco membership determined by Luhman and collaborators.
} 
sure that we had that region of parameter space wellrepresented here.

As a result of the larger areal coverage and the $\mathrm{G}$ star deficit, we sought to reassess membership for stars with K2 LCs that were not already in the highest-quality members, in particular, stars meeting those criteria. We investigated the literature for each possible additional target in question, as well as the Gaia DR2 data. In most cases, a star had several or many papers in the literature citing it as a suspected Taurus member, and it had DR2 data consistent with belonging to Taurus, or it had very few references suggesting membership and usually DR2 data inconsistent with membership. The less likely (lower-confidence) members include targets added by our own additional star-bystar inspections, including a few from Galli et al. (2019) or Kraus et al. (2017) that were not in Esplin \& Luhman (2019).

In this process, we realized that there was a subset of stars that had indications of youth but distances and/or motions incompatible with Taurus membership; these include those "controversial" members mentioned above. Many of these targets are listed in Luhman (2018) as Group 29 members (see also Oh et al. 2018). Some appear in the literature as possible members of Mu Tau (M. Gagne et al. 2020, in preparation; Liu et al. 2020) or possibly Cas-Tau (e.g., Hartmann et al. 1991; de Zeeuw et al. 1999; Luhman 2018; also see appendix of David et al. 2018). Analyses to date of this population in the literature suggest that they are $30 \mathrm{Myr}$ (e.g., Kraus et al. 2017). Our list of Taurus foreground stars are primarily those Group 29 members, plus targets added by our own additional star-by-star inspections that might very well be $\mathrm{Mu}$ Tau or Cas-Tau members (see also Stauffer et al. 2020).

Much of the supporting non-survey data available in the literature (Section 2.3) is biased toward those least controversial members, and those targets nearby in projected distance. For example, a high fraction of the highest-confidence members have a Spitzer and/or Herschel counterpart
(Sections 2.3 and 2.4; Table 1) because those pointed missions targeted the most well-known members. Circularly, the most reliable members also are more likely to have an IR excess and, thus, be detected in various IR data sets. Similarly, we expect members to be young and significantly spotted and, therefore, periodic more often, which is the case (Section 3.1).

\section{A.2. Nonmembers}

After extracting the members (of any sort) out of the $\sim 850$ in our initial sample, we are left with $\sim 630$ nominal NMs. Among this nominal NM sample, there is an anomalously high fraction of apparent IR excesses and an anomalously high periodic fraction (Table 1). Looking at the distribution of Gaia distances from Bailer-Jones et al. (2018), many are nominally at $\sim 140 \mathrm{pc}$, more than would be expected for a randomly selected set of field stars. We suspect that we have been somewhat conservative in identifying members, and that some (perhaps several) legitimate members are left in the NM sample. Moreover, the set of stars with K2 LCs is biased toward objects appearing in the literature as young, which makes the entire K2 sample different than any random selection of stars.

For these NMs, we performed much of the same analysis as for our members, but they were largely ignored in the discussion in the rest of the paper. We include results from these additional stars here, should they be of use to future investigators. We provide the NMs in Table A1, which has the same contents as in Table 2 above (except for the columns on cluster membership and dippers/bursters, none of which are found among the NMs). Note that the Gaia DR2 ID is provided for easily matching parallaxes and proper motions. We focused on the analysis of the members, and as such, some of the NMs may not have been vetted as closely as the member sample. 
Table A1

Periods and Supporting Data for Taurus Likely Nonmembers with K2 Light Curves

\begin{tabular}{|c|c|c|}
\hline Number & Label & Contents \\
\hline 1 & EPIC & Number in the Ecliptic Plane Input Catalog (EPIC) for K2 \\
\hline 2 & Coord & R.A. and decl. (J2000) for target \\
\hline 3 & OName & Alternate name for target \\
\hline 4 & Gaia & Gaia DR2 ID \\
\hline 5 & $V \mathrm{mag}$ & $V$ magnitude (in Vega mags), if observed \\
\hline 6 & $K s$ mag & $K_{\mathrm{s}}$ magnitude (in Vega mags), if observed \\
\hline 7 & $(V-K s) \mathrm{o}$ & $\left(V-K_{\mathrm{s}}\right)$, as directly observed (in Vega mags), if $V$ and $K_{\mathrm{s}}$ exist \\
\hline 8 & $(V-K s) \mathrm{u}$ & $\left(V-K_{\mathrm{s}}\right)$ used, in Vega mags (observed or inferred; see the text) \\
\hline 9 & $E(V-K s)$ & $E\left(V-K_{s}\right)$ adopted for this star (in mags; see Section 2.5) \\
\hline 10 & Ks mag0 & dereddened $K_{s, 0}$ magnitude (in Vega mags), as inferred (see Section 2.5) \\
\hline 11 & $(V-K s) 0$ & $\left(V-K_{s}\right)_{0}$, dereddened $V-K_{s}$ (in Vega mags), as inferred (see Section 2.5 ; rounded to nearest 0.1 to emphasize the relatively low accuracy) \\
\hline 12 & Uncert & 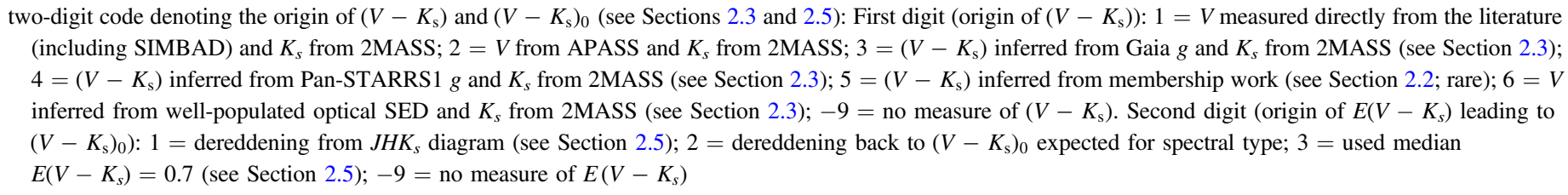 \\
\hline 13 & P1 & Primary period, in days (taken to be rotation period in cases where there is $>1$ period) \\
\hline 14 & P2 & Secondary period, in days \\
\hline 15 & P3 & Tertiary period, in days \\
\hline 16 & P4 & Quaternary period, in days \\
\hline 17 & IR excess & Whether an IR excess is present or not (see Section 4) \\
\hline 18 & IRexStr & Minimum wavelength at which the IR excess is detected or the limit of our knowledge of where there is no excess (see Section 4) \\
\hline 19 & Slope & Slope of SED fit to all available detections between 2 and $25 \mu \mathrm{m}$ \\
\hline 20 & Class & SED class (I, flat, II, III) \\
\hline 21 & Sin/mult & single or multi-period star \\
\hline 22 & dd & LC and power spectrum matches double-dip characteristics (see Section 6.2) \\
\hline 23 & ddmove & LC and power spectrum matches moving double-dip characteristics (see Section 6.2) \\
\hline 24 & Shape & LC matches shape changer characteristics (see Section 6.2) \\
\hline 25 & Beat & LC has beating visible (see Section 6.2) \\
\hline 26 & Complex & power spectrum has a complex, structured peak and/or has a wide peak (see Section 6.2) \\
\hline 27 & Closep & power spectrum has resolved close peaks (see Section 6.2) \\
\hline 28 & Distp & power spectrum has resolved distant peaks (see Section 6.2) \\
\hline 29 & Pulse & power spectrum and LC match pulsator characteristics (see Section 6.2) \\
\hline
\end{tabular}

(This table is available in its entirety in machine-readable form.) 
Table B1

Lists of Objects with Timescales

\begin{tabular}{lccc}
\hline \hline EPIC & Other Name & Membership & Timescale \\
\hline 210552148 & LP415-113 & NM & $\sim 35$ \\
210666196 & $\ldots$ & NM & $\sim 15$ \\
210683818 & HBC 393 & Mem & $\sim 25$ \\
210716897 & RXJ 0426.3+1836 & NM & $\sim 38^{\text {a }}$ \\
246835564 & $\ldots$ & NM & $\sim 28$ \\
247122185 & $\ldots$ & NM & $\sim 35$ \\
247532735 & $V^{*}$ V1108 Tau & NM & $\sim 15$ \\
247575425 & IRAS 04303+2240 & Mem & $\sim 7.5$ \\
247590222 & IRAS 04302+2247 & Mem & $\sim 25$ \\
247805410 & V*FX Tau & Mem & $\sim 11.6$ \\
247820507 & RRA 2004]Haro 6-10VLA1 & Mem & $\sim 13.6$ \\
247946210 & IRASF 04570+2520 & NM & $\sim 35$ \\
247961728 & $V^{*}$ V414 Tau & NM & $\sim 35$ \\
248019700 & SS Ttau 044325.1+255706 & NM & $\sim 23$ \\
248049475 & $V^{*}$ DO Tau & Mem & $\sim 16$ \\
248054085 & SSTtau 044453.1+261257 & NM & $\sim 16.5$ \\
\hline
\end{tabular}

Note.

a This star has an additional real, shorter period, which we take to be the rotation period.

\section{Appendix B \\ Timescales}

As in Papers I-V, some LCs have some repeated patterns that we cannot identify with certainty as a rotation period. These "timescales" tend to be longer than most of the rotation periods. Sometimes, there is not enough data to go $>1$ complete cycle. Table B1 summarizes the timescales for the stars out of the entire ensemble. Note that some also appear in the list of periodic stars but with a shorter period that we believe to be the rotation period; the longer-term variability is unlikely to be rotation.

\section{Appendix C Comparison to Literature Periods}

In this appendix section, we compare periods for stars in common between our study and several literature studies, regardless of whether or not the stars are currently thought to be Taurus members or not. Specific stars are listed in Table C1.

Rebull et al. (2004) collected periods from the then-current literature for many young stars, including stars in Taurus. There are 27 stars in common between the literature compilation in Rebull et al. (2004) and the present study, but only 23 of them are periodic in both studies; see Figure C1. Most (17) of them match periods to within a fractional difference of $10 \%$, leaving six that do not match. In three cases (EPIC $210690735=$ UX Tau), $247592463=$ HP Tau, $248029373=$ DK Tau), the LC is complex enough that it could explain the different period obtained in the literature. For EPIC 210670948, the period compiled in Rebull et al. (2004; citing Preibisch \& Smith 1997) does not match other literature nor the K2 period, and we conclude that it is wrong. For 246770655, the period from the literature matches our secondary period (though the primary period appears in the Figure). In the last case, EPIC 247034775 (V1076 Tau), the literature reports conflicting values. Rebull et al. (2004) recorded the period as 6.2 days, apparently originally from Grankin (1993), and that is what appears in Figure C1 and Table C2. Grankin (1997) reports that the period of 9.91 days from Grankin (1994) "is more credible." The K2 data yield $P=9.5412$ days, supporting the longer period; there is no $\sim 6$ days period in the $\mathrm{K} 2$ data.

Grankin et al. (2007) presented periods for 39 stars in Taurus obtained as part of the ROTOR program at Mount Maidanak Observatory. Eighteen of those stars also have K2 LCs that we determine to be periodic (four more are not periodic in $\mathrm{K} 2$ ). Most (12) of these stars do not have the same periods in the K2 data. Five of them have very different periods, and five more are plausibly harmonics; see Figure C1. As part of this same ROTOR project, Artemenko et al. (2012) published several additional periods. Of the seven new stars in common, all of them have complex LCs. Only four have periods we derived from the $\mathrm{K} 2$ data; three of them match very well, and the fourth may be a harmonic.

As part of the XMM-Newton Extended Survey of the Taurus Molecular Cloud (XEST; Güdel et al. 2007) and the Spitzer Taurus survey (Rebull et al. 2010), an extensive survey of the literature at that time was made, and a list of periods assembled to which we can compare. There are 40 stars in common with our study, only 29 of which are periodic in both studies. However, most of those are curiously listed as limits on the period in Güdel et al. (2007) see Figure C1. For those stars with secure periods, there is a good match, eight of the nine are well within $10 \%$.

Xiao et al. (2012) used the Trans-Atlantic Exoplanet Survey (TrES) to monitor L1495, a portion of the Taurus molecular cloud. Only three stars are in common between the studies: EPICs 247822311 (WK 812), 248029373 (DK Tau), and 248040905 (IQ Tau). EPIC 248029373 is the only one where the periods are not effectively identical. We obtain 7.84 days where Xiao et al. report 4.094 days, which is not consistent with our data. However, the K2 LC is complex and it is plausible that another campaign at a different time may obtain a longer period.

Rigon et al. (2017) used The Wide Angle Search for Planets (WASP) to probe the long-term variability of stars largely in Taurus-Auriga. Only two stars are in common, AA Tau (EPIC 247810494; also see below) and VO Tau (EPIC 248049475). Rigon et al. report period ranges, and we do not find periods for either one. For the first one, they report a 58 and a 5 day period; other literature report 8-13 days periods. For the latter, they report 5-6, 24, and 6-7 days. We see both of these stars as irregular bursters during the $\mathrm{K} 2$ window and cannot determine a period; we would not be sensitive to periods longer than $\sim 35$ days in any case.

Rodriguez et al. (2017a) used the KELT to find rotation periods (and dippers) in Taurus. Separately, the KELT LC for EPIC $248180268=$ HD $283782=$ V1334 Tau appeared in Rodriguez et al. (2017b); since there is a period reported there that came from KELT data, we include this period here. In total, there are 33 stars in common between us, 26 of which are periodic in both studies. Only seven match periods to within $10 \%$; see Figure C1. A few more have periods in KELT comparable to our secondary or tertiary period; see Table C2. For each of the stars in common, the LCs were investigated in detail, and the KELT period could not be recovered in most cases. We find that a few stars appear as irregular bursters (at least during the $\mathrm{K} 2$ epoch), so perhaps it is not surprising in those cases that the periods are so different. There is no clear reason why the rest of the stars are not recovered at the same periods. However, many of the KELT LCs that do not match are of very low amplitude, and the KELT LCs cover a very 
Table C1

Comparison to Literature Periods (All in Units of Days)

\begin{tabular}{|c|c|c|c|c|c|c|c|c|c|c|c|c|c|c|c|}
\hline EPIC & Other Name & $\begin{array}{l}\text { Rebull } \\
+04\end{array}$ & $\begin{array}{l}\text { Grankin } \\
\quad+07\end{array}$ & $\begin{array}{l}\text { Artemenko } \\
\quad+12\end{array}$ & $\begin{array}{l}\text { Güdel } \\
+07\end{array}$ & $\begin{array}{l}\text { Xiao } \\
+17\end{array}$ & $\begin{array}{l}\text { Rodriguez } \\
\quad+17 \mathrm{~b}\end{array}$ & $\begin{array}{l}\text { Scholz } \\
+18\end{array}$ & $\begin{array}{l}\text { Hambálek } \\
\quad+19\end{array}$ & $P_{1}$ & $P_{2}$ & $P_{3}$ & $P_{4}$ & Match? & Notes \\
\hline 210631263 & HD 285372 & 0.573 & $\ldots$ & $\ldots$ & $\ldots$ & $\ldots$ & $\ldots$ & $\ldots$ & $\ldots$ & 0.5733 & $\ldots$ & $\ldots$ & $\ldots$ & $\mathrm{y}$ & $\ldots$ \\
\hline 210662824 & HD 285778 & 2.74 & $\ldots$ & $\ldots$ & $\ldots$ & $\ldots$ & $\ldots$ & $\ldots$ & 2.736 & 2.7503 & 1.3713 & $\ldots$ & $\ldots$ & $\mathrm{y}$ & $\ldots$ \\
\hline 210670948 & $\begin{array}{c}{[\text { FK 83] }} \\
\text { LDN155151 }\end{array}$ & 1.2 & $\cdots$ & $\cdots$ & 2.43 & $\cdots$ & $\cdots$ & $\cdots$ & $\cdots$ & 2.4282 & $\cdots$ & $\cdots$ & $\cdots$ & $\mathrm{y} / \mathrm{n}$ & $\begin{array}{l}\text { Match with Güdel+ but not } \\
\text { Rebull+. }\end{array}$ \\
\hline 210674635 & $\begin{array}{l}\text { 2MASS } \\
\text { J04312405 } \\
+1800215\end{array}$ & $\cdots$ & $\cdots$ & $\cdots$ & $<6.29$ & $\cdots$ & $\cdots$ & $\cdots$ & $\cdots$ & 2.2098 & $\cdots$ & $\cdots$ & $\cdots$ & $\mathrm{n}$ & $\begin{array}{l}\text { True that } P_{\text {here }} \text { is }<P_{\text {XEST, }} \\
\text { but } 2.2 \text { days really clear in } \\
\text { K2, and nothing at } \sim 6 \text { days. }\end{array}$ \\
\hline 210683818 & HBC 393 & $\cdots$ & $\cdots$ & $\cdots$ & $<3.82$ & $\cdots$ & $\cdots$ & $\cdots$ & $\cdots$ & $\cdots$ & $\cdots$ & $\cdots$ & $\cdots$ & $\mathrm{n}$ & $\begin{array}{c}\text { At best, K2 data suggest a } \\
20-25 \text { days timescale; there } \\
\text { is not anything near } \\
3.8 \text { days. }\end{array}$ \\
\hline 210689130 & HD 28150 & $\ldots$ & 0.696 & $\cdots$ & $\ldots$ & $\cdots$ & $\ldots$ & $\cdots$ & $\ldots$ & 0.6981 & $\ldots$ & $\ldots$ & $\ldots$ & $\mathrm{y}$ & $\ldots$ \\
\hline 210690598 & EM*LkHA358 & $\cdots$ & $\cdots$ & $\cdots$ & $<6.8$ & $\cdots$ & $\cdots$ & $\cdots$ & $\cdots$ & 3.5045 & 3.7378 & $\cdots$ & $\cdots$ & $\mathrm{n}$ & $\begin{array}{l}\text { True that } P_{\text {here }} \text { is }<P_{\mathrm{XEST}}, \\
\text { but } \sim 3 \text { days really clear in } \\
\mathrm{K} 2, \text { and nothing at } \sim 7 \text { days. }\end{array}$ \\
\hline 210690735 & $\mathrm{~V}^{*} \mathrm{UX} \mathrm{Tau}$ & 2.7 & $\cdots$ & $\cdots$ & $\cdots$ & $\cdots$ & $\cdots$ & $\cdots$ & $\cdots$ & 3.6584 & $\cdots$ & $\cdots$ & $\cdots$ & $\mathrm{n}$ & $\begin{array}{l}\text { No match with Rebull+ } \\
\text { from either period. How- } \\
\text { ever, K2 LC power spec- } \\
\text { trum suggests } \sim 2 \text { days at } \\
\text { another time may be } \\
\text { plausible. }\end{array}$ \\
\hline 210690892 & XZ Tau AB & 2.6 & $\cdots$ & 3.24 & 2.6 & $\cdots$ & $\cdots$ & $\cdots$ & $\ldots$ & $\ldots$ & $\cdots$ & $\ldots$ & $\cdots$ & $\mathrm{n}$ & No period in K2. \\
\hline 210690913 & $\mathrm{~V}^{*} \mathrm{HL} \mathrm{Tau}$ & $\cdots$ & $\cdots$ & $\cdots$ & $<7.21$ & $\cdots$ & $\cdots$ & $\cdots$ & $\cdots$ & $\cdots$ & $\cdots$ & $\cdots$ & $\cdots$ & $\mathrm{n}$ & $\begin{array}{c}\text { There is a } 7.7 \text { days peak in } \\
\text { the K2 data but it does not } \\
\text { phase well; a } 24 \text { days period } \\
\text { is more plausible in the } \\
\text { K2 data. }\end{array}$ \\
\hline 210698143 & $\begin{array}{l}\text { 2MASS } \\
\text { J04311578 } \\
+1820072\end{array}$ & $\cdots$ & $\cdots$ & $\cdots$ & $<7.66$ & $\cdots$ & $\cdots$ & $\cdots$ & $\cdots$ & 1.5954 & 3.4961 & $\cdots$ & $\cdots$ & $\mathrm{n}$ & $\begin{array}{l}\text { True that } P_{\text {here }} \text { is }<P_{\text {XEST, }} \\
\text { but periods really clear in } \\
\mathrm{K} 2 \text {, and nothing at } \sim 7 \text { days. }\end{array}$ \\
\hline 210698281 & V*V827 Tau & 3.75 & $\cdots$ & $\cdots$ & 3.75 & $\cdots$ & $\cdots$ & $\cdots$ & $\cdots$ & 3.7582 & $\cdots$ & $\cdots$ & $\cdots$ & $\mathrm{y}$ & $\cdots$ \\
\hline 210699801 & EM*LkHA267 & $\ldots$ & $\cdots$ & $\cdots$ & $<5.67$ & $\cdots$ & $\cdots$ & $\cdots$ & $\cdots$ & 4.3047 & 1.07 & $\cdots$ & $\cdots$ & $\mathrm{n}$ & $\begin{array}{c}\text { True that } P_{\text {here }} \text { is }<P_{\mathrm{XEST}} \text {, } \\
\text { but periods clear in } \mathrm{K} 2 \text { and } \\
\text { nothing at } \sim 5.6 \text { days. }\end{array}$ \\
\hline 210767482 & HD 284418 & $\ldots$ & $\ldots$ & $\ldots$ & $\ldots$ & $\ldots$ & 14.81 & $\ldots$ & $\ldots$ & 15.3682 & $\ldots$ & $\ldots$ & $\ldots$ & $\mathrm{y}$ & $\sim$ match \\
\hline 210777988 & $\mathrm{~V}^{*} \mathrm{~T}$ Tau & 2.8 & $\ldots$ & $\ldots$ & 2.8 & $\ldots$ & $\ldots$ & $\ldots$ & $\ldots$ & 2.8116 & $\ldots$ & $\ldots$ & $\ldots$ & $\mathrm{y}$ & $\ldots$ \\
\hline 210792668 & $\ldots$ & $\cdots$ & 1.168 & $\cdots$ & $\ldots$ & $\cdots$ & $\cdots$ & $\cdots$ & $\ldots$ & $\ldots$ & $\cdots$ & $\ldots$ & $\ldots$ & $\mathrm{n}$ & No period in $\mathrm{K} 2$. \\
\hline 210805120 & $\mathrm{~V}^{*} \mathrm{~V} 1196 \mathrm{Tau}$ & 3.02 & $\ldots$ & $\cdots$ & $\ldots$ & $\ldots$ & $\ldots$ & $\cdots$ & $\ldots$ & $\ldots$ & $\ldots$ & $\ldots$ & $\cdots$ & $\mathrm{y}$ & No period in $\mathrm{K} 2$. \\
\hline 210818897 & V*V1298 Tau & $\ldots$ & 2.86 & $\ldots$ & $\ldots$ & $\ldots$ & $\ldots$ & $\ldots$ & 2.885 & 2.8484 & 1.4508 & $\ldots$ & $\ldots$ & $\mathrm{y}$ & $\ldots$ \\
\hline 210881343 & & $\cdots$ & 0.736 & $\cdots$ & $\cdots$ & $\cdots$ & $\cdots$ & $\cdots$ & $\cdots$ & 6.4388 & 2.7789 & $\cdots$ & $\cdots$ & $\mathrm{n}$ & $\begin{array}{c}\text { No period near } 0.7 \text { in } \\
\text { K2 data. }\end{array}$ \\
\hline 210977750 & $\begin{array}{c}\text { 2MASS } \\
\mathrm{J} 04073502 \\
+2237394\end{array}$ & $\cdots$ & 0.816 & $\cdots$ & $\cdots$ & $\cdots$ & $\cdots$ & $\cdots$ & $\cdots$ & 0.1346 & 0.1507 & $\cdots$ & $\cdots$ & $\mathrm{n}$ & $\begin{array}{c}\text { No period near } 0.8 \text { in } \\
\text { K2 data. }\end{array}$ \\
\hline 211104793 & $\begin{array}{c}\text { 2MASS } \\
\mathrm{J} 04124068 \\
+2438157\end{array}$ & $\cdots$ & 5.58 & $\ldots$ & $\ldots$ & $\cdots$ & $\cdots$ & $\cdots$ & $\cdots$ & 5.8543 & $\cdots$ & $\cdots$ & $\cdots$ & $\mathrm{y}$ & $\cdots$ \\
\hline
\end{tabular}


Table C1

(Continued)

\begin{tabular}{|c|c|c|c|c|c|c|c|c|c|c|c|c|c|c|c|}
\hline EPIC & Other Name & $\begin{array}{l}\text { Rebull } \\
+04\end{array}$ & $\begin{array}{l}\text { Grankin } \\
\quad+07\end{array}$ & $\begin{array}{l}\text { Artemenko } \\
\quad+12\end{array}$ & $\begin{array}{l}\text { Güdel } \\
+07\end{array}$ & $\begin{array}{l}\text { Xiao } \\
+17\end{array}$ & $\begin{array}{l}\text { Rodriguez } \\
\quad+17 \mathrm{~b}\end{array}$ & $\begin{array}{l}\text { Scholz } \\
+18\end{array}$ & $\begin{array}{l}\text { Hambálek } \\
\quad+19\end{array}$ & $P_{1}$ & $P_{2}$ & $P_{3}$ & $P_{4}$ & Match? & Notes \\
\hline 211182143 & $\ldots$ & $\ldots$ & 1.961 & $\ldots$ & $\cdots$ & $\cdots$ & $\ldots$ & $\ldots$ & 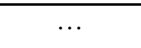 & $\cdots$ & $\cdots$ & $\cdots$ & $\ldots$ & $\mathrm{n}$ & No period in K2. \\
\hline 246695532 & $\mathrm{~V}^{*} \mathrm{~V} 1840$ Ori & $\cdots$ & 1.231 & $\cdots$ & $\cdots$ & $\cdots$ & $\cdots$ & $\cdots$ & $\cdots$ & 5.343 & $\cdots$ & $\cdots$ & $\cdots$ & $\mathrm{n}$ & $\begin{array}{l}\text { There is a peak near } 1.5 \\
\text { days in one and only one of } \\
\text { the data reductions } \\
\text { (K2SFF); we suspect that } \\
\text { this period may correspond } \\
\text { to one of the other targets } \\
\text { near } 246695532 .\end{array}$ \\
\hline 246760205 & HD 286179 & 3.33 & $\cdots$ & $\cdots$ & $\cdots$ & $\cdots$ & $\cdots$ & $\cdots$ & 3.14 & 3.1297 & $\cdots$ & $\cdots$ & $\cdots$ & $\mathrm{y}$ & $\ldots$ \\
\hline 246770655 & $\begin{array}{l}\text { WDS J04573 } \\
+1524 \mathrm{AB}\end{array}$ & 2.39 & $\cdots$ & $\cdots$ & $\cdots$ & $\cdots$ & $\cdots$ & $\cdots$ & 2.412 & 1.7013 & 2.3551 & $\cdots$ & $\cdots$ & $\mathrm{y}$ & $\sim$ match with our $P_{2}$ \\
\hline 246798563 & $\mathrm{~V}^{*} \mathrm{~V} 1326 \mathrm{Tau}$ & $\ldots$ & 2.54 & $\ldots$ & $\ldots$ & $\ldots$ & $\ldots$ & $\ldots$ & $\ldots$ & 2.5385 & $\ldots$ & $\ldots$ & $\ldots$ & $\mathrm{y}$ & $\ldots$ \\
\hline 246802680 & HD 285957 & 3.07 & $\ldots$ & $\ldots$ & $\ldots$ & $\ldots$ & $\ldots$ & $\ldots$ & 3.055 & 3.0868 & $\ldots$ & $\ldots$ & $\ldots$ & $\mathrm{y}$ & $\ldots$ \\
\hline 246815623 & $\mathrm{~V}^{*} \mathrm{~V} 1351 \mathrm{Tau}$ & $\ldots$ & 5.64 & $\ldots$ & $\ldots$ & $\ldots$ & $\ldots$ & $\ldots$ & $\ldots$ & 5.6313 & $\ldots$ & $\ldots$ & $\ldots$ & $\mathrm{y}$ & $\ldots$ \\
\hline 246825172 & $\mathrm{~V}^{*} \mathrm{~V} 1353 \mathrm{Tau}$ & $\cdots$ & 0.884 & $\cdots$ & $\cdots$ & $\cdots$ & $\cdots$ & $\cdots$ & $\cdots$ & 7.6307 & $\cdots$ & $\cdots$ & $\cdots$ & $\mathrm{n}$ & $\begin{array}{l}\text { No period near } 0.9 \text { days in } \\
\text { K2 data. }\end{array}$ \\
\hline 246923113 & $\mathrm{~V}^{*} \mathrm{DR} \mathrm{Tau}$ & 2.8 & $\cdots$ & $\cdots$ & $\cdots$ & $\cdots$ & $\cdots$ & $\cdots$ & $\cdots$ & 14.5698 & $\cdots$ & $\cdots$ & $\cdots$ & $\mathrm{n}$ & $\begin{array}{l}\text { No period near } 2.8 \text { days in } \\
\text { K2 data. }\end{array}$ \\
\hline 246977631 & V*V1346 Tau & $\cdots$ & 0.829 & $\cdots$ & $\cdots$ & $\cdots$ & $\cdots$ & $\cdots$ & $\cdots$ & 4.6167 & $\cdots$ & $\cdots$ & $\cdots$ & $\mathrm{n}$ & $\begin{array}{l}\text { No period near } 0.8 \text { days in } \\
\text { K2 data. }\end{array}$ \\
\hline 247031423 & HD 28867 & $\ldots$ & $\ldots$ & $\cdots$ & $<2$ & $\ldots$ & $\cdots$ & $\cdots$ & $\ldots$ & 3.0978 & 3.2775 & $\cdots$ & $\cdots$ & $\mathrm{n}$ & $\begin{array}{l}\text { No match even with the } \\
\text { inequality; perhaps Güedel } \\
\quad+\text { found a harmonic? }\end{array}$ \\
\hline 247032616 & $\mathrm{~V}^{*} \mathrm{~V} 826 \mathrm{Tau}$ & 3.7 & $\ldots$ & $\ldots$ & 3.7 & $\ldots$ & $\ldots$ & $\ldots$ & $\ldots$ & 3.8829 & $\ldots$ & $\ldots$ & $\ldots$ & $\mathrm{y}$ & $\ldots$ \\
\hline 247034775 & $\mathrm{~V}^{*} \mathrm{~V} 1076 \mathrm{Tau}$ & 6.2 & $\cdots$ & $\cdots$ & $\ldots$ & $\cdots$ & $\cdots$ & $\cdots$ & $\cdots$ & 9.5412 & $\cdots$ & $\cdots$ & $\cdots$ & $\mathrm{n}$ & $\begin{array}{l}\text { No period near } 6 \text { days in } \mathrm{K} 2 \\
\text { data, but } 6 \text { days may not be } \\
\text { real; } 9.91 \text { days from Gran- } \\
\text { kin (1994) is a better match } \\
\text { to the K2 data. }\end{array}$ \\
\hline 247047380 & $\mathrm{~V}^{*} \mathrm{DM}$ Tau & $\cdots$ & $\cdots$ & $\cdots$ & $<6.74$ & $\cdots$ & $\cdots$ & $\ldots$ & $\cdots$ & 7.4081 & $\ldots$ & $\ldots$ & $\cdots$ & $\mathrm{n}$ & $\begin{array}{c}\text { Not a match, but the K2 LC } \\
\text { has potentially many peri- } \\
\text { ods in it, including near } \\
6 \text { days. }\end{array}$ \\
\hline 247051861 & $\begin{array}{c}2 \text { MASS } \\
\text { J04321606 } \\
+1812464\end{array}$ & $\cdots$ & $\cdots$ & $\cdots$ & $<7.84$ & $\cdots$ & $\cdots$ & $\cdots$ & $\cdots$ & 2.6496 & $\cdots$ & $\ldots$ & $\cdots$ & $\mathrm{n}$ & $\begin{array}{l}\text { True that } P_{\text {here }} \text { is }<P_{\mathrm{XEST}}, \\
\text { but } \sim 3 \text { days really clear in } \\
\mathrm{K} 2, \text { and nothing at } \sim 8 \text { days. }\end{array}$ \\
\hline 247076294 & HD 31281 & $\ldots$ & $\ldots$ & $\ldots$ & $\ldots$ & $\ldots$ & $\ldots$ & $\ldots$ & 0.791 & 0.6771 & 0.7999 & $\ldots$ & $\ldots$ & $\mathrm{y}$ & Hambalek + found our $P_{2}$. \\
\hline 247119725 & [WKS 96]42 & $\cdots$ & $\cdots$ & $\cdots$ & $\cdots$ & $\cdots$ & 1.31 & $\cdots$ & $\cdots$ & 4.175 & $\cdots$ & $\cdots$ & $\cdots$ & $\mathrm{n}$ & $\begin{array}{l}\text { No period near } 1.3 \text { days in } \\
\text { K2 data. }\end{array}$ \\
\hline 247126197 & HD 285840 & 1.55 & $\ldots$ & $\ldots$ & $\ldots$ & $\ldots$ & $\ldots$ & $\ldots$ & 1.548 & 1.5461 & $\ldots$ & $\ldots$ & $\ldots$ & $\mathrm{y}$ & $\ldots$ \\
\hline 247139505 & LP 415-183 & $\ldots$ & 1.41 & $\cdots$ & $\cdots$ & $\cdots$ & $\cdots$ & $\cdots$ & $\ldots$ & 18.3841 & $\cdots$ & $\ldots$ & $\cdots$ & $\mathrm{n}$ & $\begin{array}{l}\text { No period near } 1.4 \text { days in } \\
\text { K2 data. }\end{array}$ \\
\hline 247225984 & V*V1333 Tau & $\ldots$ & $\ldots$ & $\ldots$ & $\ldots$ & $\ldots$ & 12.594 & $\ldots$ & $\ldots$ & 12.9213 & $\ldots$ & $\ldots$ & $\ldots$ & $\mathrm{y}$ & $\ldots$ \\
\hline 247280905 & $\mathrm{~V}^{*} \mathrm{~V} 1325 \mathrm{Tau}$ & $\ldots$ & 2.96 & $\ldots$ & $\ldots$ & $\ldots$ & 2.935 & $\ldots$ & $\ldots$ & 3.9332 & 2.9233 & 11.7995 & $\ldots$ & $\mathrm{y}$ & Others match our $P_{2}$. \\
\hline 247303990 & & $\cdots$ & 1.46 & $\ldots$ & $\cdots$ & $\cdots$ & $\ldots$ & $\cdots$ & $\ldots$ & 0.5498 & 0.793 & $\ldots$ & $\cdots$ & $\mathrm{n}$ & $\begin{array}{l}\text { Complicated K2 LC, with } \\
\text { several strong peaks, but }\end{array}$ \\
\hline
\end{tabular}


Table C1

(Continued)

\begin{tabular}{|c|c|c|c|c|c|c|c|c|c|c|c|c|c|c|c|}
\hline EPIC & Other Name & $\begin{array}{c}\text { Rebull } \\
+04\end{array}$ & $\begin{array}{l}\text { Grankin } \\
\quad+07\end{array}$ & $\begin{array}{l}\text { Artemenko } \\
\quad+12\end{array}$ & $\begin{array}{l}\text { Güdel } \\
+07\end{array}$ & $\begin{array}{l}\text { Xiao } \\
+17\end{array}$ & $\begin{array}{l}\text { Rodriguez } \\
\quad+17 \mathrm{~b}\end{array}$ & $\begin{array}{l}\text { Scholz } \\
+18\end{array}$ & $\begin{array}{l}\text { Hambálek } \\
\quad+19\end{array}$ & $P_{1}$ & $P_{2}$ & $P_{3}$ & $P_{4}$ & Match? & Notes \\
\hline & $\begin{array}{c}\text { 2MASS } \\
\text { J04564525 } \\
+2035116\end{array}$ & & & & & & & & & & & & & & $\begin{array}{l}\text { none of them are at } \sim 1.5 \\
\text { days, and none of them are } \\
\text { harmonics of } 1.5 \text { days. }\end{array}$ \\
\hline 247360583 & $\begin{array}{l}\text { 2MASS } \\
\text { J05064662 } \\
+2104296\end{array}$ & $\cdots$ & 1.079 & $\cdots$ & $\cdots$ & $\cdots$ & $\cdots$ & $\cdots$ & $\cdots$ & 0.4389 & $\cdots$ & $\cdots$ & $\cdots$ & $\mathrm{n}$ & $\begin{array}{l}\text { No period near } 1.1 \text { days in } \\
\text { K2 data. }\end{array}$ \\
\hline 247454835 & HD 284496 & 2.71 & $\ldots$ & $\ldots$ & $\ldots$ & $\ldots$ & 1.593 & $\ldots$ & 2.688 & 2.7733 & 2.653 & $\ldots$ & $\ldots$ & $\mathrm{y} / \mathrm{n}$ & Rodriguez + is the outlier. \\
\hline 247520207 & EM*LkCa15 & 5.85 & $\ldots$ & $\ldots$ & $\ldots$ & $\ldots$ & 5.765 & $\ldots$ & $\ldots$ & 5.7795 & $\ldots$ & $\ldots$ & $\ldots$ & $\mathrm{y}$ & $\ldots$ \\
\hline 247528573 & $\begin{array}{c}\text { 2MASS } \\
\text { J04464475 } \\
+2224508\end{array}$ & $\cdots$ & $\cdots$ & $\cdots$ & $\cdots$ & $\cdots$ & 0.517 & $\cdots$ & $\cdots$ & $\cdots$ & $\cdots$ & $\cdots$ & $\cdots$ & $\mathrm{n}$ & No period in $\mathrm{K} 2$. \\
\hline 247539775 & $\mathrm{~V}^{*} \mathrm{~V} 1341 \mathrm{Tau}$ & $\cdots$ & 0.478 & $\cdots$ & $\cdots$ & $\cdots$ & 11.198 & $\cdots$ & $\cdots$ & 0.9165 & $\cdots$ & $\cdots$ & $\cdots$ & $\mathrm{n}$ & $\begin{array}{l}\text { Very, very clear period in } \\
\mathrm{K} 2 \text {, not near } 0.5 \text { or } 11 \text { days. }\end{array}$ \\
\hline 247548866 & $\begin{array}{l}\text { [BLH 2002] } \\
\text { KPNO-Tau8 }\end{array}$ & $\cdots$ & $\cdots$ & $\cdots$ & $\cdots$ & $\cdots$ & $\cdots$ & 0.69 & $\cdots$ & 0.6878 & $\cdots$ & $\cdots$ & $\cdots$ & $\mathrm{y}$ & $\cdots$ \\
\hline 247575425 & $\begin{array}{c}\text { IRAS } \\
04303+2240\end{array}$ & $\cdots$ & $\cdots$ & $\cdots$ & $<5.23$ & $\cdots$ & $\cdots$ & $\cdots$ & $\cdots$ & $\cdots$ & $\cdots$ & $\cdots$ & $\cdots$ & $\mathrm{n}$ & $\begin{array}{l}\text { Complicated K2 LC power } \\
\text { spectrum suggests } \sim 5 . \mathrm{x} \\
\text { days at another time may be } \\
\text { plausible. }\end{array}$ \\
\hline 247575958 & $\begin{array}{c}\text { 2MASS } \\
\mathrm{J} 04330945 \\
+2246487\end{array}$ & $\ldots$ & $\cdots$ & $\cdots$ & $\cdots$ & $\cdots$ & $\cdots$ & 3.51 & $\cdots$ & 3.4916 & $\cdots$ & $\cdots$ & $\cdots$ & $\mathrm{y}$ & $\cdots$ \\
\hline 247584113 & CI Tau & $\cdots$ & $\cdots$ & 16.1 & $<9.2$ & $\cdots$ & $\cdots$ & $\cdots$ & $\cdots$ & 9.0335 & 6.6224 & $\cdots$ & $\cdots$ & $\mathrm{n}$ & $\begin{array}{l}\text { More } P \text { in literature are } \\
8.9891,16.10 \text { (Johns-Krull } \\
\text { et al. 2016). } \sim 9 \text { days is } \\
\text { thought to be period of pla- } \\
\text { net, which is recovered; } \\
\text { nothing near } 16 \text { days in K2, } \\
\text { but LC complicated. More } P \\
\text { in literature (based on this } \\
\text { K2 LC) is } \sim 6.6 \text { and } \sim 9, \text { for } \\
\text { star and planet, respectively } \\
\text { (Biddle et al. 2018). }\end{array}$ \\
\hline 247585953 & $\begin{array}{l}\text { 2MASS } \\
\text { J04340717 } \\
+2251227\end{array}$ & $\cdots$ & $\cdots$ & $\cdots$ & $\cdots$ & $\cdots$ & 1.177 & $\cdots$ & $\cdots$ & 2.9775 & 5.3517 & 1.4534 & $\cdots$ & $\mathrm{n}$ & $\begin{array}{l}\text { No period near } 1.7 \text { days in } \\
\text { K2 data. }\end{array}$ \\
\hline 247591534 & $\begin{array}{c}\text { 2MASS } \\
\text { J04355760 } \\
+2253574\end{array}$ & $\cdots$ & $\cdots$ & $\cdots$ & $\cdots$ & $\cdots$ & $\cdots$ & 1.16 & $\cdots$ & 1.1984 & $\cdots$ & $\cdots$ & $\cdots$ & $\mathrm{y}$ & $\cdots$ \\
\hline 247592103 & CoKuHP TauG2 & 1.2 & $\cdots$ & $\cdots$ & 1.2 & $\cdots$ & 5.798 & $\cdots$ & $\cdots$ & 1.1978 & 1.222 & $\cdots$ & $\cdots$ & $\mathrm{y} / \mathrm{n}$ & Rodriguez + is the outlier. \\
\hline 247592463 & V*HP Tau & 5.9 & $\cdots$ & $\cdots$ & 5.9 & $\cdots$ & $\cdots$ & $\cdots$ & $\cdots$ & 4.3307 & $\cdots$ & $\cdots$ & $\cdots$ & $\mathrm{n}$ & $\begin{array}{l}\text { Complex K2 LC, could } \\
\text { explain mismatch. }\end{array}$ \\
\hline 247592919 & Haro 6-28 & $\cdots$ & $\cdots$ & $\cdots$ & $<3.98$ & $\cdots$ & $\cdots$ & $\cdots$ & $\cdots$ & $\cdots$ & $\cdots$ & $\cdots$ & $\cdots$ & $\mathrm{n}$ & $\begin{array}{l}\text { Complex } \mathrm{K} 2 \mathrm{LC} \text {, no clear } \\
\text { periods. }\end{array}$ \\
\hline 247594260 & $\mathrm{~V}^{*} \mathrm{~V} 1335 \mathrm{Tau}$ & $\cdots$ & 3.762 & $\cdots$ & $\cdots$ & $\cdots$ & 3.766 & $\cdots$ & $\cdots$ & 3.7998 & $\cdots$ & $\cdots$ & $\cdots$ & $\mathrm{y}$ & $\ldots$ \\
\hline 247599080 & $\begin{array}{c}\text { IRAS } \\
04295+2251\end{array}$ & $\cdots$ & $\cdots$ & $\cdots$ & $<1.48$ & $\cdots$ & $\cdots$ & $\cdots$ & $\cdots$ & 1.7081 & $\cdots$ & $\ldots$ & $\ldots$ & $\mathrm{n}$ & \\
\hline
\end{tabular}


Table C1

(Continued)

\begin{tabular}{|c|c|c|c|c|c|c|c|c|c|c|c|c|c|c|c|}
\hline EPIC & Other Name & $\begin{array}{l}\text { Rebull } \\
+04\end{array}$ & $\begin{array}{l}\text { Grankin } \\
\quad+07\end{array}$ & $\begin{array}{l}\text { Artemenko } \\
\quad+12\end{array}$ & $\begin{array}{l}\text { Güdel } \\
+07\end{array}$ & $\begin{array}{l}\text { Xiao } \\
+17\end{array}$ & $\begin{array}{l}\text { Rodriguez } \\
\quad+17 \mathrm{~b}\end{array}$ & $\begin{array}{l}\text { Scholz } \\
+18\end{array}$ & $\begin{array}{l}\text { Hambálek } \\
+19\end{array}$ & $P_{1}$ & $P_{2}$ & $P_{3}$ & $P_{4}$ & Match? & Notes \\
\hline & & & & & & & & & & & & & & & $\begin{array}{c}\text { No match even with the } \\
\text { inequality; K2 LC is } \\
\text { low SNR. }\end{array}$ \\
\hline 247600777 & $\begin{array}{c}2 \text { MASS } \\
\text { J04363893 } \\
+2258119\end{array}$ & $\cdots$ & $\cdots$ & $\cdots$ & $\cdots$ & $\cdots$ & $\cdots$ & 0.96 & $\cdots$ & 0.9645 & $\cdots$ & $\cdots$ & $\cdots$ & $\mathrm{y}$ & $\ldots$ \\
\hline 247604448 & $\begin{array}{c}\text { 2MASS } \\
\text { J04361038 } \\
+2259560\end{array}$ & $\cdots$ & $\cdots$ & $\cdots$ & $\cdots$ & $\cdots$ & $\cdots$ & 2.91 & $\cdots$ & 2.933 & $\cdots$ & $\cdots$ & $\cdots$ & $\mathrm{y}$ & $\cdots$ \\
\hline 247609913 & $\mathrm{~V}^{*} \mathrm{~V} 1117 \mathrm{Tau}$ & $\cdots$ & $\cdots$ & $\cdots$ & $\cdots$ & $\cdots$ & 1.184 & $\cdots$ & $\cdots$ & 6.3887 & $\cdots$ & $\cdots$ & $\cdots$ & $\mathrm{n}$ & $\begin{array}{l}\text { No period near } 1.2 \text { days in } \\
\text { K2 data. }\end{array}$ \\
\hline 247630187 & $\begin{array}{c}\text { 2MASS } \\
\text { J04350850 } \\
+2311398\end{array}$ & $\cdots$ & $\cdots$ & $\cdots$ & $\cdots$ & $\cdots$ & $\cdots$ & 1.5 & $\cdots$ & 1.4981 & $\cdots$ & $\cdots$ & $\cdots$ & $\mathrm{y}$ & $\ldots$ \\
\hline 247739445 & $\begin{array}{c}\text { 2MASS } \\
\text { J04302365 } \\
+2359129\end{array}$ & $\cdots$ & $\cdots$ & $\cdots$ & $\cdots$ & $\cdots$ & $\cdots$ & 1.61 & $\cdots$ & 1.6119 & $\cdots$ & $\cdots$ & $\cdots$ & $\mathrm{y}$ & $\cdots$ \\
\hline 247748412 & $\begin{array}{c}2 \mathrm{MASS} \\
\mathrm{J} 04322329 \\
+2403013\end{array}$ & $\cdots$ & $\cdots$ & $\cdots$ & $\cdots$ & $\cdots$ & $\cdots$ & 3.37 & $\cdots$ & 3.3643 & $\cdots$ & $\cdots$ & $\cdots$ & $\mathrm{y}$ & $\cdots$ \\
\hline 247763883 & $\mathrm{~V}^{*} \mathrm{GH} \mathrm{Tau}$ & $\ldots$ & $\ldots$ & $\ldots$ & $<3.57$ & $\ldots$ & $\ldots$ & $\ldots$ & $\ldots$ & 2.4937 & 2.9408 & $\cdots$ & $\cdots$ & $\mathrm{n}$ & $\begin{array}{l}\text { True that } P_{\text {here }} \text { is }<P_{\mathrm{XEST}} \\
\text { K2 LC power spectrum } \\
\text { suggests a longer period at } \\
\text { another time may be } \\
\text { plausible. }\end{array}$ \\
\hline 247764745 & $\mathrm{~V}^{*} \mathrm{~V} 807 \mathrm{Tau}$ & $\cdots$ & $\cdots$ & $\cdots$ & $\cdots$ & $\cdots$ & 0.809 & $\cdots$ & $\cdots$ & 4.3784 & $\ldots$ & $\cdots$ & $\cdots$ & $\mathrm{n}$ & $\begin{array}{l}\text { No period near } 0.8 \text { days in } \\
\text { K2 data. }\end{array}$ \\
\hline 247776236 & HD 28975 & $\cdots$ & $\cdots$ & $\cdots$ & $\cdots$ & $\cdots$ & 0.957 & $\cdots$ & $\cdots$ & 4.353 & $\cdots$ & $\cdots$ & $\cdots$ & $\mathrm{n}$ & $\begin{array}{l}\text { No period near } 0.9 \text { days in } \\
\text { K2 data. }\end{array}$ \\
\hline 247781229 & HD 28819 & $\cdots$ & $\cdots$ & $\cdots$ & $\cdots$ & $\cdots$ & 0.516 & $\cdots$ & $\ldots$ & 0.6086 & 0.2849 & $\cdots$ & $\cdots$ & $\mathrm{n}$ & $\begin{array}{l}\text { K2 LC power spectrum } \\
\text { suggests that } \sim 0.5 \text { days at } \\
\text { another time may be } \\
\text { plausible. }\end{array}$ \\
\hline 247791556 & $\begin{array}{c}\text { 2MASS } \\
\text { J04330197 } \\
+2421000\end{array}$ & $\cdots$ & $\cdots$ & $\cdots$ & $<4.67$ & $\cdots$ & $\cdots$ & 1.03 & $\cdots$ & 1.0261 & 1.0469 & $\cdots$ & $\cdots$ & $\mathrm{n}$ & $\begin{array}{l}\text { True that } P_{\text {here }} \text { is }<P_{\mathrm{XEST}} \\
\text { nothing near } 4.6 \text { days in } \mathrm{K} 2 .\end{array}$ \\
\hline 247791801 & $\mathrm{~V}^{*} \mathrm{GK}$ Tau & 4.6 & $\ldots$ & 4.61 & 4.6 & $\ldots$ & $\ldots$ & $\cdots$ & $\ldots$ & 4.6165 & $\ldots$ & $\ldots$ & $\ldots$ & $\mathrm{y}$ & $\ldots$ \\
\hline 247792225 & V*GI Tau & 7.2 & $\cdots$ & 7.09 & 7.2 & $\cdots$ & $\cdots$ & $\cdots$ & $\ldots$ & 7.1334 & .. & $\ldots$ & $\cdots$ & $\mathrm{y}$ & $\ldots$ \\
\hline 247795097 & $\mathrm{~V}^{*} \mathrm{~V} 928 \mathrm{Tau}$ & $\cdots$ & $\cdots$ & $\cdots$ & $<7.45$ & $\cdots$ & $\cdots$ & $\cdots$ & $\cdots$ & 2.2468 & 2.4876 & $\cdots$ & $\cdots$ & $\mathrm{n}$ & $\begin{array}{c}\text { True that } P_{\text {here }} \text { is }<P_{\text {XEST; }} \\
\text { K2 periods very clear and } \\
\text { nothing near } 7 \text { days. }\end{array}$ \\
\hline 247799571 & $\mathrm{~V}^{*} \mathrm{HK} \mathrm{Tau}$ & $\cdots$ & $\cdots$ & $\cdots$ & $<8.86$ & $\cdots$ & $\cdots$ & $\cdots$ & $\cdots$ & 3.3056 & $\cdots$ & $\cdots$ & $\cdots$ & $\mathrm{n}$ & $\begin{array}{l}\text { True that } P_{\text {here }} \text { is }<P_{\mathrm{XEST}} \\
\text { K2 LC power spectrum } \\
\text { suggests a longer period at } \\
\text { another time may be } \\
\text { plausible. }\end{array}$ \\
\hline 247804500 & & $\cdots$ & $\cdots$ & $\cdots$ & $\cdots$ & $\cdots$ & 0.929 & $\cdots$ & $\cdots$ & $\cdots$ & $\cdots$ & $\cdots$ & $\ldots$ & $\mathrm{n}$ & No period in $\mathrm{K} 2$. \\
\hline
\end{tabular}


Table C1

(Continued)

\begin{tabular}{|c|c|c|c|c|c|c|c|c|c|c|c|c|c|c|c|}
\hline EPIC & Other Name & $\begin{array}{l}\text { Rebull } \\
+04\end{array}$ & $\begin{array}{l}\text { Grankin } \\
\quad+07\end{array}$ & $\begin{array}{l}\text { Artemenko } \\
\quad+12\end{array}$ & $\begin{array}{l}\text { Güdel } \\
+07\end{array}$ & $\begin{array}{l}\text { Xiao } \\
+17\end{array}$ & $\begin{array}{l}\text { Rodriguez } \\
\quad+17 \mathrm{~b}\end{array}$ & $\begin{array}{l}\text { Scholz } \\
+18\end{array}$ & $\begin{array}{l}\text { Hambálek } \\
+19\end{array}$ & $P_{1}$ & $P_{2}$ & $P_{3}$ & $P_{4}$ & Match? & Notes \\
\hline & $\begin{array}{c}\text { 2MASS } \\
\mathrm{J} 04352474 \\
+2426218\end{array}$ & & & & & & & & & & & & & & \\
\hline 247805410 & $\mathrm{~V}^{*} \mathrm{FX} \mathrm{Tau}$ & $\cdots$ & $\cdots$ & $\cdots$ & $<12.34$ & $\cdots$ & $\cdots$ & $\cdots$ & $\cdots$ & $\cdots$ & $\cdots$ & $\cdots$ & $\cdots$ & $\mathrm{n}$ & $\begin{array}{l}\text { Complicated K2 LC power } \\
\text { spectrum suggests a longer } \\
\text { period at another time may } \\
\text { be plausible. }\end{array}$ \\
\hline 247810494 & $\mathrm{~V}^{*} \mathrm{AA} \mathrm{Tau}$ & 8.22 & $\cdots$ & 8.19 & 8.22 & $\cdots$ & 13.613 & $\cdots$ & $\cdots$ & $\cdots$ & $\cdots$ & $\cdots$ & $\cdots$ & $\mathrm{y} / \mathrm{n}$ & $\begin{array}{l}\text { Rigon et al. (2017) reports } \\
58,5 \text { days. No period in K2, } \\
\text { but LC has a lot of structure, } \\
\text { suggesting that periodic } \\
\text { behavior at another time } \\
\text { may be plausible. }\end{array}$ \\
\hline 247810751 & Haro 6-13 & $\cdots$ & $\cdots$ & $\cdots$ & $<7.3$ & $\cdots$ & $\cdots$ & $\cdots$ & $\cdots$ & 3.2778 & $\cdots$ & $\cdots$ & $\cdots$ & $\mathrm{n}$ & $\begin{array}{l}\text { True that } P_{\text {here }} \text { is }<P_{\mathrm{XEST}} \\
\text { K2 LC power spectrum } \\
\text { suggests a longer period at } \\
\text { another time may be } \\
\text { plausible. }\end{array}$ \\
\hline 247820507 & $\begin{array}{c}\text { [RRA 2004] } \\
\text { Haro 6-10VLA1 }\end{array}$ & $\cdots$ & $\cdots$ & $\cdots$ & $<5.63$ & $\cdots$ & $\cdots$ & $\cdots$ & $\cdots$ & $\cdots$ & $\cdots$ & $\cdots$ & $\cdots$ & $\mathrm{n}$ & $\begin{array}{l}\text { No match even with the } \\
\text { inequality; K } 2 \text { LC power } \\
\text { spectrum suggests a shorter } \\
\text { period at another time may } \\
\text { be plausible. }\end{array}$ \\
\hline 247822311 & WK 812 & 2.75 & $\cdots$ & $\cdots$ & 2.75 & 2.747 & 2.743 & $\cdots$ & $\cdots$ & 2.7412 & $\cdots$ & $\cdots$ & $\cdots$ & $\mathrm{y}$ & $\cdots$ \\
\hline 247837468 & $\begin{array}{c}\text { IRAS } \\
04264+2433\end{array}$ & $\cdots$ & $\cdots$ & $\cdots$ & $<1.52$ & $\cdots$ & $\cdots$ & $\cdots$ & $\cdots$ & 11.8717 & $\cdots$ & $\cdots$ & $\cdots$ & $\mathrm{n}$ & $\begin{array}{l}\text { No match even with the } \\
\text { inequality; K2 LC power } \\
\text { spectrum suggests a shorter } \\
\text { period at another time may } \\
\text { be plausible. }\end{array}$ \\
\hline 247843485 & $\mathrm{~V}^{*} \mathrm{ZZ}$ Tau & $\cdots$ & $\cdots$ & $\cdots$ & $\cdots$ & $\cdots$ & 1.311 & $\cdots$ & $\cdots$ & 4.1609 & $\cdots$ & $\cdots$ & $\cdots$ & $\mathrm{n}$ & $\begin{array}{l}\text { No period near } 1.3 \text { days in } \\
\text { K2 data. }\end{array}$ \\
\hline 247864498 & V*IW Tau & 5.6 & $\cdots$ & $\cdots$ & $\cdots$ & $\cdots$ & 0.844 & $\cdots$ & $\cdots$ & 5.5013 & 7.0425 & $\cdots$ & $\cdots$ & $\mathrm{y} / \mathrm{n}$ & Rodriguez + is the outlier. \\
\hline 247915927 & $\begin{array}{c}\text { IRAS } \\
\text { S04414+2506 }\end{array}$ & $\cdots$ & $\cdots$ & $\cdots$ & $\cdots$ & $\cdots$ & $\cdots$ & 4.48 & $\cdots$ & 4.43 & $\cdots$ & $\cdots$ & $\cdots$ & $\mathrm{y}$ & $\cdots$ \\
\hline 247923794 & $\mathrm{~V}^{*} \mathrm{DP} \mathrm{Tau}$ & $\cdots$ & $\cdots$ & $\cdots$ & $<2.76$ & $\cdots$ & $\cdots$ & $\cdots$ & $\cdots$ & 3.6623 & $\cdots$ & $\cdots$ & $\cdots$ & $\mathrm{n}$ & $\begin{array}{l}\text { No match even with the } \\
\text { inequality; K2 LC power } \\
\text { spectrum suggests a shorter } \\
\text { period at another time may } \\
\text { be plausible. }\end{array}$ \\
\hline 247935061 & $\mathrm{~V}^{*} \mathrm{GO} \mathrm{Tau}$ & $\cdots$ & $\cdots$ & $\cdots$ & $<3.96$ & $\cdots$ & $\cdots$ & $\cdots$ & $\cdots$ & $\cdots$ & $\cdots$ & $\cdots$ & $\cdots$ & $\mathrm{n}$ & $\begin{array}{l}\text { No period in } \mathrm{K} 2 \text {, but } \mathrm{LC} \text { has } \\
\text { a lot of structure, suggesting } \\
\text { that periodic behavior at } \\
\text { another time may be } \\
\text { plausible. }\end{array}$ \\
\hline 247941378 & V*V999 Tau & $\cdots$ & $\cdots$ & $\cdots$ & $<2.25$ & $\cdots$ & 23.935 & $\cdots$ & $\cdots$ & 4.9052 & 2.6515 & $\ldots$ & $\cdots$ & $\mathrm{n}$ & \\
\hline
\end{tabular}


Table C1

(Continued)

\begin{tabular}{|c|c|c|c|c|c|c|c|c|c|c|c|c|c|c|c|}
\hline EPIC & Other Name & $\begin{array}{c}\text { Rebull } \\
+04\end{array}$ & $\begin{array}{l}\text { Grankin } \\
+07\end{array}$ & $\begin{array}{l}\text { Artemenko } \\
\quad+12\end{array}$ & $\begin{array}{c}\text { Güdel } \\
+07\end{array}$ & $\begin{array}{r}\text { Xiao } \\
+17\end{array}$ & $\begin{array}{l}\text { Rodriguez } \\
\quad+17 \mathrm{~b}\end{array}$ & $\begin{array}{l}\text { Scholz } \\
+18\end{array}$ & $\begin{array}{l}\text { Hambálek } \\
+19\end{array}$ & $P_{1}$ & $P_{2}$ & $P_{3}$ & $P_{4}$ & Match? & Notes \\
\hline & & & & & & & & & & & & & & & $\begin{array}{l}\text { Two K2 periods are very } \\
\text { clear, neither consistent with } \\
\text { prior observations. }\end{array}$ \\
\hline 247941613 & $\mathrm{~V}^{*} \mathrm{~V} 1000 \mathrm{Tau}$ & $\cdots$ & $\cdots$ & $\cdots$ & $\cdots$ & $\cdots$ & 23.935 & $\cdots$ & $\cdots$ & 2.1764 & 8.2537 & $\cdots$ & $\cdots$ & $\mathrm{n}$ & $\begin{array}{l}\text { Complex K2 LC, could } \\
\text { explain mismatch. }\end{array}$ \\
\hline 247941930 & EM*LkHA332 & $\cdots$ & $\cdots$ & $\cdots$ & $<4.9$ & $\cdots$ & 23.935 & $\cdots$ & $\cdots$ & $\cdots$ & $\cdots$ & $\cdots$ & $\cdots$ & $\mathrm{n}$ & $\begin{array}{l}\text { Complex K2 LC, could be } \\
\text { plausible at another time. }\end{array}$ \\
\hline 247950452 & $\begin{array}{c}\text { 2MASS } \\
\text { J04334291 } \\
+2526470\end{array}$ & $\cdots$ & $\cdots$ & $\cdots$ & $\cdots$ & $\cdots$ & $\cdots$ & 0.73 & $\cdots$ & 0.7269 & $\cdots$ & $\cdots$ & $\cdots$ & $\mathrm{y}$ & $\cdots$ \\
\hline 247953586 & $\begin{array}{c}2 \text { MASS } \\
\text { J04320329 } \\
+2528078\end{array}$ & $\cdots$ & $\cdots$ & $\cdots$ & $\cdots$ & $\cdots$ & $\cdots$ & 2.39 & $\cdots$ & 2.3765 & $\cdots$ & $\cdots$ & $\cdots$ & $\mathrm{y}$ & $\cdots$ \\
\hline 247968420 & $\begin{array}{c}\text { 2MASS } \\
\text { J04414825 } \\
+2534304\end{array}$ & $\cdots$ & $\cdots$ & $\cdots$ & $\cdots$ & $\cdots$ & $\cdots$ & 2.9 & $\cdots$ & 2.9144 & $\cdots$ & $\cdots$ & $\cdots$ & $\mathrm{y}$ & $\cdots$ \\
\hline 247986526 & DF Tau AB & 7.2 & $\cdots$ & 7.18 & $\cdots$ & $\cdots$ & 16.504 & $\cdots$ & $\cdots$ & $\cdots$ & $\cdots$ & $\cdots$ & $\cdots$ & $\mathrm{n}$ & $\begin{array}{l}\text { Complex K2 LC, could be } \\
\text { plausible at another time. }\end{array}$ \\
\hline 247991214 & $\begin{array}{c}\text { 2MASS } \\
\text { J04390396 } \\
+2544264\end{array}$ & $\cdots$ & $\cdots$ & $\cdots$ & $\cdots$ & $\cdots$ & $\cdots$ & 3.3 & $\cdots$ & 3.1401 & $\cdots$ & $\cdots$ & $\cdots$ & $\mathrm{y}$ & $\cdots$ \\
\hline 247992574 & $\mathrm{~V}^{*} \mathrm{GN} \mathrm{Tau}$ & $\cdots$ & $\cdots$ & $\cdots$ & $<11.8$ & $\cdots$ & $\cdots$ & $\cdots$ & $\cdots$ & 5.7452 & $\cdots$ & $\cdots$ & $\cdots$ & $\mathrm{n}$ & $\begin{array}{l}\text { True that } P_{\text {here }} \text { is }<P_{\mathrm{XEST}} \\
\text { LC has a lot of structure, } \\
\text { suggesting that a different } \\
\text { period at another time may } \\
\text { be plausible. }\end{array}$ \\
\hline 248009353 & UZ Tau AB & $\cdots$ & $\cdots$ & $\cdots$ & $<4.9$ & $\cdots$ & $\cdots$ & $\cdots$ & $\cdots$ & 3.7527 & $\cdots$ & $\cdots$ & $\cdots$ & $\mathrm{n}$ & $\begin{array}{l}\text { True that } P_{\text {here }} \text { is }<P_{\mathrm{XEST}} \\
\text { LC has a lot of structure, } \\
\text { suggesting that a different } \\
\text { period at another time may } \\
\text { be plausible. }\end{array}$ \\
\hline 248010721 & HD 283707 & $\cdots$ & $\cdots$ & $\cdots$ & $\cdots$ & $\cdots$ & 0.967 & $\cdots$ & $\cdots$ & $\cdots$ & $\ldots$ & $\ldots$ & $\ldots$ & $\mathrm{n}$ & No period in $\mathrm{K} 2$. \\
\hline 248014510 & HD 283809 & $\cdots$ & $\cdots$ & $\cdots$ & $\cdots$ & $\cdots$ & 20.121 & $\cdots$ & $\cdots$ & 1.653 & 1.4033 & 0.9651 & 2.3834 & $\mathrm{n}$ & $\begin{array}{l}\text { No period near } 20 \text { days in } \\
\text { K2 data. }\end{array}$ \\
\hline 248018164 & Haro 6-33 & $\cdots$ & $\cdots$ & $\cdots$ & $<2.13$ & $\cdots$ & $\cdots$ & $\cdots$ & $\cdots$ & $\cdots$ & $\cdots$ & $\cdots$ & $\cdots$ & $\mathrm{n}$ & $\begin{array}{l}\text { No period in K2, but LC has } \\
\text { a lot of structure, suggesting } \\
\text { that periodic behavior at } \\
\text { another time may be } \\
\text { plausible. }\end{array}$ \\
\hline 248018652 & $\begin{array}{l}\text { [BLH 2002] } \\
\text { KPNO-Tau7 }\end{array}$ & $\cdots$ & $\cdots$ & $\cdots$ & $\cdots$ & $\cdots$ & $\cdots$ & 1.18 & $\cdots$ & 1.1572 & $\cdots$ & $\cdots$ & $\cdots$ & $\mathrm{y}$ & $\cdots$ \\
\hline 248019693 & XEST02-040 & $\cdots$ & 3.12 & $\cdots$ & $\cdots$ & $\cdots$ & $\cdots$ & $\cdots$ & $\cdots$ & 1.4708 & $\cdots$ & $\cdots$ & $\cdots$ & $\mathrm{n}$ & $\begin{array}{c}\text { Strong EB in K2 data, no } P \\
\text { at } 3 \text { days. }\end{array}$ \\
\hline 248023915 & $\begin{array}{l}\text { 2MASS } \\
\text { J04380083 } \\
+2558572\end{array}$ & $\cdots$ & $\cdots$ & $\cdots$ & $\cdots$ & $\cdots$ & $\cdots$ & 2 & $\cdots$ & 0.6644 & 1.0263 & $\cdots$ & $\cdots$ & $\mathrm{n}$ & $\begin{array}{c}\text { No period near } 2 \text { days in } \\
\text { K2 data. }\end{array}$ \\
\hline 248029373 & DK Tau AB & 8.4 & $\cdots$ & 8.18 & $\cdots$ & 4.094 & $\cdots$ & $\cdots$ & $\cdots$ & 7.8414 & $\cdots$ & $\cdots$ & $\cdots$ & $\mathrm{n}$ & \\
\hline
\end{tabular}




\begin{tabular}{|c|c|c|c|c|c|c|c|c|c|c|c|c|c|c|c|}
\hline EPIC & Other Name & $\begin{array}{c}\text { Rebull } \\
+04\end{array}$ & $\begin{array}{l}\text { Grankin } \\
\quad+07\end{array}$ & $\begin{array}{l}\text { Artemenko } \\
\quad+12\end{array}$ & $\begin{array}{l}\text { Güdel } \\
+07\end{array}$ & $\begin{array}{r}\text { Xiao } \\
+17\end{array}$ & $\begin{array}{l}\text { Rodriguez } \\
\quad+17 \mathrm{~b}\end{array}$ & $\begin{array}{l}\text { Scholz } \\
+18\end{array}$ & $\begin{array}{l}\text { Hambálek } \\
+19\end{array}$ & $P_{1}$ & $P_{2}$ & $P_{3}$ & $P_{4}$ & Match? & Notes \\
\hline & & & & & & & & & & & & & & & $\begin{array}{l}\text { Complex K2 LC, could } \\
\text { explain mismatch. }\end{array}$ \\
\hline 248029954 & $\begin{array}{c}\text { 2MASS } \\
\text { J04394748 }\end{array}$ & $\cdots$ & $\cdots$ & $\cdots$ & $\cdots$ & $\cdots$ & $\cdots$ & 2.9 & $\cdots$ & 2.9502 & $\cdots$ & $\cdots$ & $\cdots$ & $\mathrm{y}$ & $\cdots$ \\
\hline
\end{tabular}

$-\mathrm{J} 04394748$
+2601407

TYC 1833-

575-1

$248049475 \quad V^{*}$ DO Tau

Table C1

Continued)

\begin{tabular}{|c|c|c|c|c|c|c|c|c|c|c|c|c|c|c|c|}
\hline & & & & & & & & & & & & & & & $\begin{array}{l}5-6,24,6-7,17,35 \text { days. } \\
\text { No period in K2, but LC has } \\
\text { a lot of structure, suggesting } \\
\text { that periodic behavior at } \\
\text { another time may be } \\
\text { plausible. }\end{array}$ \\
\hline 248055184 & IT Tau A & $\ldots$ & $\ldots$ & $\ldots$ & $\ldots$ & $\ldots$ & 2.751 & $\ldots$ & $\ldots$ & 2.7434 & $\ldots$ & $\ldots$ & $\ldots$ & $\mathrm{y}$ & $\ldots$ \\
\hline 248060724 & $\begin{array}{c}\text { [BLH 2002] } \\
\text { KPNO-Tau14 }\end{array}$ & $\cdots$ & $\cdots$ & $\cdots$ & $\cdots$ & $\cdots$ & $\ldots$ & 1.86 & $\cdots$ & 1.8637 & $\cdots$ & $\cdots$ & $\cdots$ & $\mathrm{y}$ & $\cdots$ \\
\hline 248145565 & HD 283798 & $\ldots$ & 0.6 & $\ldots$ & $\ldots$ & $\ldots$ & 0.965 & $\ldots$ & 0.987 & 0.9831 & 0.9658 & $\ldots$ & $\cdots$ & $y / n$ & Grankin+ is the outlier. \\
\hline 248175684 & $\mathrm{~V}^{*} \mathrm{~V} 1328 \mathrm{Tau}$ & $\cdots$ & $\cdots$ & $\cdots$ & $\cdots$ & $\cdots$ & 0.601 & $\cdots$ & $\cdots$ & 1.5287 & 3.8492 & $\cdots$ & $\cdots$ & $\mathrm{n}$ & $\begin{array}{l}\text { No period near } 0.6 \text { days in } \\
\text { K2 data. }\end{array}$ \\
\hline 248180268 & HD 283782 & $\cdots$ & $\ldots$ & $\ldots$ & $\cdots$ & $\cdots$ & 0.321 & $\ldots$ & 0.87 & 0.465 & 0.3214 & 2.0182 & $\cdots$ & $\mathrm{n}$ & $\begin{array}{l}\text { No period near } 0.8 \text { days in } \\
\text { K2 data; Rodriguez }+ \text { mat- } \\
\text { ches our } P_{2} \text {. }\end{array}$ \\
\hline 248245414 & $\cdots$ & $\cdots$ & 0.921 & $\cdots$ & $\cdots$ & $\cdots$ & $\cdots$ & $\cdots$ & $\cdots$ & $\cdots$ & $\ldots$ & $\cdots$ & $\cdots$ & $\mathrm{n}$ & $\begin{array}{l}\text { No period near } 0.9 \text { days in } \\
\text { K2 data. }\end{array}$ \\
\hline 248248731 & $\cdots$ & $\cdots$ & 2.43 & $\cdots$ & $\cdots$ & $\cdots$ & $\cdots$ & $\cdots$ & $\cdots$ & $\cdots$ & $\cdots$ & $\cdots$ & $\cdots$ & $\mathrm{n}$ & $\begin{array}{l}\text { No period near } 2 \text { days in } \\
\text { K2 data. }\end{array}$ \\
\hline
\end{tabular}



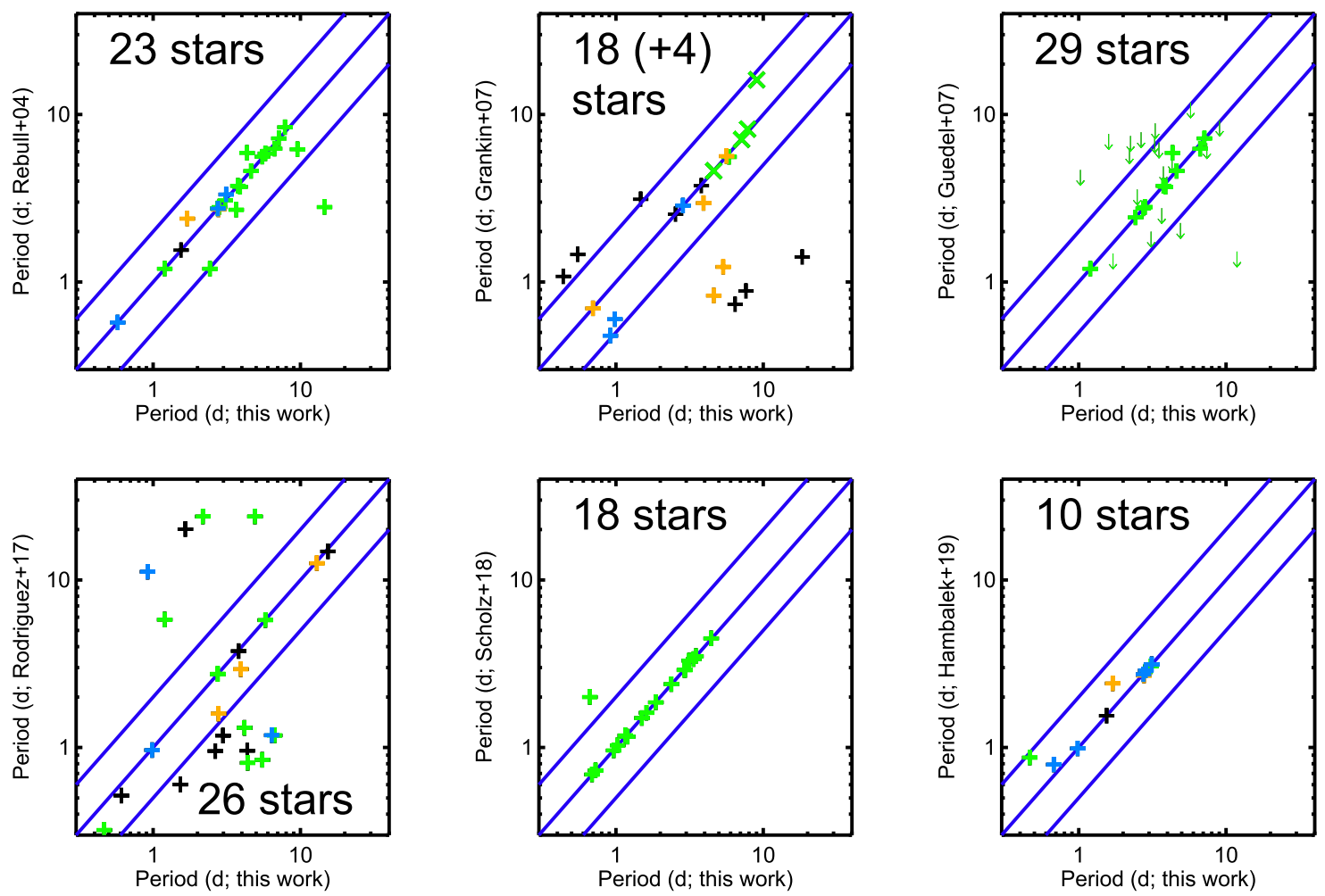

Figure C1. Comparison of periods obtained here to periods obtained in the literature. The colors of the points are as follows: green $=$ highest-confidence member; orange $=$ possible member; blue $=$ young, foreground object; and black = likely NM (see Section 2.2). From the upper left to lower right panels: Rebull et al (2004), literature compilation, 23 periodic stars in common; Grankin et al. (2007), 18 periodic stars in common, with four more (shown as $\times$ ) from Artemenko et al. (2012), Güdel et al. (2007), literature compilation, 29 periodic stars in common; Rodriguez et al. (2017a), KELT, 26 periodic stars in common; Scholz et al. (2018), K2, 18 stars in common; Hambálek et al. (2019), SuperWASP/NSVS/K2, 10 stars in common. The dark blue lines are at one-to-one, $P / 2$, and 2P. (Xiao et al. 2012 has only three stars in common and so is not plotted; see the text and Table C2. Rigon et al. (2017) reports period ranges and so is not plotted here.) Most of the periods match well (see the text for much more discussion).

long time baseline, so if, say, periods are appearing/ disappearing at different latitudes under the assumption of surface differential rotation, the rotation period may appear to change over the KELT baseline, making it harder to determine. For the LCs we have, our periods are correct, though some of the LCs are complex. (We note that our recovery rate between $\mathrm{K} 2$ and KELT was much higher in our earlier K2 papers.)

More recently, Scholz et al. (2018) used some of the same $\mathrm{K} 2$ data as we are using to explore the rotation rates of brown dwarfs in Taurus, and there are 18 stars in common. The periods are all recovered well, except that of EPIC 248023915 (2MASS J04380083+2558572), where the period reported in Scholz et al. is about twice the second period we obtain; we suspect they found an alias rather than the true period.

Most recently, Hambálek et al. (2019) used SuperWASP, the Northern Sky Variability Survey (NSVS), and K2 to study 20 weak-lined $\mathrm{T}$ Tauri stars in Taurus; there are 12 stars in common, 10 of which are periodic in both studies. Most of the stars are well matched; see Figure C1. There are three stars where we report two K2 periods, and Hambálek et al. find the period we identified as the second period: EPICs 247076294, 248180268 , and 246770655 . In the case of 247076294 , the two $\mathrm{K} 2$ periods are so close to each other that the difference does not stand out in the plot, but the Hambálek et al. period matches the second $\mathrm{K} 2$ period.

AA Tau (EPIC 247810494) is somewhat of a special case. It has been the subject of numerous, intensive studies (e.g., Bouvier et al. 1999, 2007; Donati et al. 2010; and references therein). Those studies identified AA Tau as having a period of
8.22 days and defining the class of "dippers" among diskbearing stars regularly occulted by disk matter close to the star. There is no evidence for an $\sim 8$ days period in the K2 data; in fact, we find no significant periodicity at all. Additionally, we find no obvious dipper signatures during this campaign; particularly in the first half of the campaign, the LC shares more characteristics with bursters than with dippers. Substantial variation has been noted in this system before (e.g., Bouvier et al. 2003, 2013). The dipper character of the LC as well as the 8.2 days period seen in ground-based LCs for decades disappeared altogether as the star underwent a major dimming event in recent years (Bouvier et al. 2013), a state from which it has not yet recovered.

\section{ORCID iDs}

L. M. Rebull (1) https://orcid.org/0000-0001-6381-515X

J. R. Stauffer (ib https://orcid.org/0000-0003-3595-7382

A. M. Cody (1) https://orcid.org/0000-0002-3656-6706

N. Roggero (1) https://orcid.org/0000-0003-4370-086X

T. J. David (1) https://orcid.org/0000-0001-6534-6246

\section{References}

Ahn, C. P., Alexandroff, R., \& Allende Prieto, C. 2012, ApJS, 203, 21

Akeson, R., Chen, X., Ciardi, D., et al. 2013, PASP, 125, 989

Artemenko, S., Grankin, K., \& Petrov, P. 2012, AstL, 38, 783

Bailer-Jones, C., Rybizki, J., Fouesneau, M., Mantelet, G., \& Andrae, R. 2018, AJ, 156, 58

Basri, G., \& Nguyen, H. 2018, ApJ, 863, 190 
Biddle, L., Johns-Krull, C., Llama, J., Prato, L., \& Skiff, B. 2018, ApJL, 853, L34

Bouvier, J., Alencar, S., Boutelier, T., et al. 2007, A\&A, 463, 1017

Bouvier, J., Chelli, A., Allain, S., et al. 1999, A\&A, 349, 619

Bouvier, J., Grangin, K., Alencar, S., et al. 2003, A\&A, 409, 169

Bouvier, J., Grankin, K., Ellerbroek, L., Bouy, H., \& Barrado, D. 2013, A\&A, 557,77

Cardelli, J., Clayton, G., \& Mathis, J. 1989, ApJ, 345, 245

Chambers, K., Magnier, E., Metcalf, N., et al. 2016, arXiv:1612.05560

Cody, A. M., \& Hillenbrand, L. A. 2018, AJ, 156, 71

Cody, A. M., Stauffer, J., Baglin, A., et al. 2014, AJ, 147, 82

Coker, C., Pinsonneault, M., \& Terndrup, D. 2016, ApJ, 833, 122

David, T., Mamajek, E., Vanderburg, A., et al. 2018, AJ, 156, 302

de Zeeuw, P., Hoogerwerf, R., de Bruijne, J., Brown, A., \& Blaauw, A. 1999, AJ, 117, 354

Donati, J.-F., Skelly, M., Bouvier, J., et al. 2010, MNRAS, 409, 1347

Esplin, T. L., \& Luhman, K. L. 2017, AJ, 154, 134

Esplin, T. L., \& Luhman, K. L. 2019, AJ, 158, 54

Gaia Collaboration, Brown, A., Vallenari, A., et al. 2016, A\&A, 595, A2

Gaia Collaboration, Brown, A., Vallenari, A., et al. 2018, A\&A, 616, A1

Galli, P. A. B., Loinard, L., Bouy, H., et al. 2019, A\&A, 630, A137

Grankin, K. N. 1993, IBVS, 3823, 1

Grankin, K. N. 1994, IBVS, 4042, 1

Grankin, K. N. 1997, AstL, 23, 615

Grankin, K. N., Artemenko, S. A., \& Melnikov, S. Y. 2007, IBVS, 5752, 1

Güdel, M., Briggs, K., Arzner, K., et al. 2007, A\&A, 468, 353

Hambálek, L., Van̂ko, M., Paunzen, E., et al. 2019, MNRAS, 483, 1642

Hartmann, L. 2002, ApJL, 566, L29

Hartmann, L., Jones, B., Stauffer, J., \& Kenyon, S. 1991, AJ, 101, 1050

Henden, A., Templeton, M., Terrell, D., et al. 2016, yCat, 2336, 0

Howell, S., et al. 2014, PASP, 126, 398

Indebetouw, R., Mathis, J., Babler, B., et al. 2005, ApJ, 619, 931

Johns-Krull, C. M., McLane, J. N., Prato, L., et al. 2016, ApJ, 826, 206

Joy, A. H. 1945, ApJ, 102, 168

Kenyon, S., \& Hartmann, L. 1995, ApJS, 101, 117

Kraus, A., Herczeg, G., Rizzuto, A., et al. 2017, ApJ, 838, 150

Kraus, A., \& Hillenbrand, L. 2009, ApJ, 704, 531

Kurucz, R. 1993, yCat, 6039, 0

Lejeune, T., Cuisinier, F., \& Buser, R. 1997, A\&AS, 125, 229

Liu, J., Fang, M., \& Liu, C. 2020, AJ, 159, 3

Luger, R., Agol, E., Kruse, E., et al. 2016, AJ, 152, 100

Luger, R., Kruse, E., Foreman-Mackey, D., Agol, E., \& Saunders, N. 2018, AJ, 156,99

Luhman, K. 2018, AJ, 156, 271

Luhman, K., Mamajek, E., Shukla, S., \& Loutrel, N. 2017, AJ, 153, 46
Marton, G., Calzoletti, L., Perez Garcia, A., et al. 2017, Explanatory Supplement, https://irsa.ipac.caltech.edu/data/Herschel/PPSC/docs/HPPSC ExplanatorySupplement.pdf

Mathis, J. 1990, ARA\&A, 28, 37

Meyer, M., Calvet, N., \& Hillenbrand, L. 1997, AJ, 114, 288

Murakami, H., Baba, H., Barthel, P., et al. 2007, PASJ, 59, 369

Nguyen, D. C., Jayawardhana, R., van Kerkwijk, M. H., et al. 2009, ApJ, 695, 1648 Oh, S., Price-Whelan, A. M., Hogg, D. W., Morton, T. D., \& Spergel, D. N. 2018, AJ, 153, 257

Pecaut, M., \& Mamajek, E. 2013, ApJS, 208, 9

Pilbratt, G., Riedinger, J., Passvogel, T., et al. 2010, A\&A, 518, L1

Preibisch, T., \& Smith, M. D. 1997, A\&A, 322, 825

Press, W., Teukolsky, S., Veterling, W., \& Flannery, B. 1992, Numerical Recipes in C (Cambridge: Cambridge Univ. Press)

Rebull, L. M., Koenig, X. P., Padgett, D. L., et al. 2011, ApJS, 196, 4

Rebull, L., Padgett, D., McCabe, C.-E., et al. 2010, ApJS, 186, 259

Rebull, L., Stauffer, J., Bouvier, J., et al. 2016a, AJ, 152, 114

Rebull, L., Stauffer, J., Cody, A., et al. 2016b, AJ, 152, 113

Rebull, L., Stauffer, J., Cody, A. M., et al. 2018, AJ, 155, 196

Rebull, L., Stauffer, J., Hillenbrand, L., et al. 2017, ApJ, 839, 92

Rebull, L., Wolff, S. C., \& Strom, S. E. 2004, AJ, 127, 1029

Rigon, L., Scholz, A., Anderson, D., \& West, R. 2017, MNRAS, 465, 3889

Rodriguez, J., Ansdell, M., Oelkers, R., et al. 2017a, ApJ, 848, 91

Rodriguez, J., Zhou, G., Cargile, P., et al. 2017b, ApJ, 836, 209

Scargle, J. D. 1982, ApJ, 263, 835

Scholz, A., Moore, K., Jayawardhana, R., et al. 2018, ApJ, 859, 153

Skrutskie, M., Cutri, R. M., Stiening, R., et al. 2006, AJ, 131, 1163

Slesnick, C., Carpenter, J., \& Hillebrand, L. 2006, AJ, 131, 3016

Stauffer, J., Barrado, D., David, T., et al. 2020, AJ, in press

Stauffer, J., Cody, A. M., Baglin, A., et al. 2014, AJ, 147, 83

Stauffer, J., Cody, A. M., McGinnis, P., et al. 2015, AJ, 149, 130

Stauffer, J., Cody, A. M., Rebull, L., et al. 2016a, AJ, 151, 60

Stauffer, J., Collier Cameron, A., Jardine, M., et al. 2017, AJ, 153, 152

Stauffer, J., Rebull, L., Bouvier, J., et al. 2016b, AJ, 152, 142

Stauffer, J., Rebull, L., Cody, A. M., et al. 2018a, AJ, 156, 275

Stauffer, J., Rebull, L., David, T., et al. 2018b, AJ, 155, 152

Tokovinin, A., \& Briceño, C. 2020, AJ, 159, 15

Vanderburg, A., \& Johnson, J. 2014, PASP, 126, 948

Werner, M., Roellig, T., Low, F., et al. 2004, ApJS, 154, 1

Wichmann, R., Krautter, J., Schmitt, J., et al. 1996, A\&A, 312, 439

Wilking, B., Bontemps, S., Schuler, R., Greene, T., \& Andrè, P. 2001, ApJ, 551,357

Wright, E., Eisenhardt, P. R. M., Mainzer, A. K., et al. 2010, AJ, 140, 1868

Xiao, H. Y., Covey, K. R., Rebull, L. M., et al. 2012, ApJS, 202, 7

Zhan, Z., Guenther, M., Rappaport, S., et al. 2019, ApJ, 876, 127 